\title{
LOGARITHMIC GEOMETRY AND ALGEBRAIC STACKS
}

\author{
BY MARTIN C. OLSSON
}

ABSTRACT. - We construct algebraic moduli stacks of log structures and give stack-theoretic interpretations of K. Kato's notions of log flat, log smooth, and log étale morphisms. In the last section we describe the local structure of these moduli stacks in terms of toric stacks.

(C) 2003 Elsevier SAS

RÉSUMÉ. - Nous construisons des champs algébriques classifiant les structures logarithmiques et donnons de nouvelles interprétations, fondées sur la théorie des champs, des concepts de morphisme log plat, log lisse, et log étale introduits par K. Kato. Dans la dernière partie, nous décrivons la structure locale de ces champs en termes de champs toriques.

() 2003 Elsevier SAS

\section{Introduction}

The purpose of this paper is to introduce a stack-theoretic approach to the theory of logarithmic geometry $[9,17]$.

Let $S$ be a fine log scheme with underlying scheme $\stackrel{\circ}{S}$, and define a fibered category

$$
\mathcal{L}_{\text {og }} \rightarrow(\stackrel{\circ}{S} \text {-schemes })
$$

as follows. The objects of $\mathcal{L}_{o g}$ are morphisms of fine log schemes $X \rightarrow S$, and a morphism $h: X_{/ S}^{\prime} \rightarrow X_{/ S}$ in $\mathcal{L}_{o g}$ is a morphism of $S$-log schemes for which $h^{b}: h^{*} \mathcal{M}_{X} \rightarrow \mathcal{M}_{X^{\prime}}$ is an

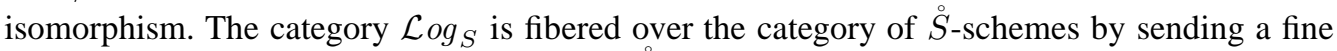
$\log$ scheme $X / S$ to the underlying scheme $\stackrel{\circ}{X}$. The main result of this paper is the following theorem, which was originally suggested to us by A. Abbes (see (1.2) for our conventions about algebraic stacks):

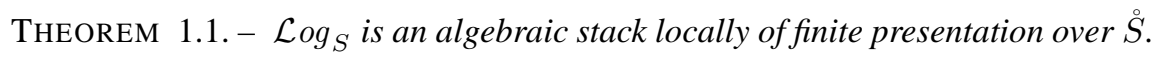

A morphism of fine log schemes $f: X \rightarrow S$ defines tautologically a morphism of algebraic stacks

$$
\mathcal{L} o g(f): \mathcal{L}_{o g} \rightarrow \mathcal{L}_{o g}
$$

and the association $S \mapsto \mathcal{L}_{\log }$ defines a 2 -functor

$$
\text { (category of log schemes) } \rightarrow(2 \text {-category of algebraic stacks) }
$$

which can be viewed as an "embedding". In this paper we explain how this 2-functor can be used to reinterpret and study basic notions in logarithmic geometry. 
The paper is organized as follows.

Section 2 contains some basic results about charts which will be used in what follows.

In Section 3 we present a proof of (1.1), assuming the result, proven in the appendix (A.2), that $\mathcal{L} g_{S}$ is a stack with respect to the fppf topology. There are two main reasons for proving that $\mathcal{L} \operatorname{og}_{S}$ is a stack with respect to the fppf topology in the appendix rather than in the main body of the paper. First, (A.2) is a corollary of a foundational result in the theory of log geometry (A.1) comparing the notion of a fine $\log$ structure in the fppf topology with the notion of a fine $\log$ structure in the étale topology. Since the proof of (A.1) is not directly related to the relationship between algebraic stacks and log geometry it seems best to prove it in an appendix. Secondly, if one restricts attention to the substack $\mathcal{T}$ or $_{S} \subset \mathcal{L} \log _{S}$ classifying fs (i.e. fine and saturated) $\log$ schemes over $S$, then the use of (A.2) can be avoided (see (3.1)). Throughout the paper we point out when results from the appendix are used, and how in the case of $\mathcal{T}$ or $r_{S}$ one can proceed without them.

The proof of (1.1) in Section 3 is based on the theory of charts developed in Section 2. We feel that this proof is the most natural for the purposes of this paper. However, one can also prove (1.1) using deformation theory of log structures and M. Artin's method [1]. In fact, Theorem (1.1) implies that a "good" deformation theory of log structures exists, and in other contexts it is useful to have an understanding of this deformation theory [18]. Closely related to this is the fact that (1.1) enables one to define the cotangent complex of a morphism of log schemes. We hope to return to this subject in the future.

Section 4 is devoted to studying properties of morphisms of log schemes $f: X \rightarrow S$ using the associated morphisms of algebraic stacks $\mathcal{L} o g(f)$. We define what it means for a morphism $f: X \rightarrow S$ of fine $\log$ schemes to have a property $\mathcal{P}$, where $\mathcal{P}$ is a property of representable morphisms of algebraic stacks. In particular, we get notions of étale, smooth, and flat morphisms of fine $\log$ schemes, and in ((4.6)-(4.7)) we show that these definitions agree with the definitions of K. Kato.

In Section 5 we explain how work of L. Illusie on "toric stacks" yields a beautiful étale cover of the stack $\log _{S}$. This enables one to describe properties of morphisms of log schemes in terms of locally defined maps to toric stacks. As in the proof of (1.1), issues about the fppf topology present themselves in this section and certain results from the appendix ((A.3)-(A.5)) are used. If one restricts attention to fs $\log$ structures, then the use of these results can be avoided, and we point out how one can do so. From the stack-theoretic point of view, however, we feel that there may be some interest in considering non-saturated log structures (see (5.29)).

In the appendix we compare the notions of fine log structure in the fppf, étale, and Zariski topology. The main result is (A.1) which asserts that if $X$ is a scheme, then there is a natural equivalence between the category of fine $\log$ structures on the fppf site $X_{\mathrm{ff}}$ and the category of fine log structures on the étale site $X_{\text {et }}$ (the comparison between fine $\log$ structures on $X_{\text {et }}$ and the Zariski site $X_{\text {Zar }}$ is slightly more complicated; see (A.1) for the statement). An immediate corollary of (A.1) is the statement mentioned above that $\mathcal{L}_{o g}$ is a stack with respect to the fppf topology (A.2). We also obtain three other corollaries ((A.3)-(A.5)) which are used in Section 5. The results about Zariski log structures are not used in the main body of the paper but are included for completeness.

Finally let us mention three applications of (1.1) which are not discussed in this paper. One can develop the theory of log crystalline cohomology using a theory of crystalline cohomology of schemes over algebraic stacks [19], and also the deformation theory of log schemes can be understood using (1.1). In addition, Theorem (1.1) has a natural place in the study of the moduli of fine log schemes [20]. We intend to discuss these subjects in future papers. 


\subsection{Conventions and prerequisites}

We assume that the reader is familiar with logarithmic geometry at the level of the first two sections of [9]. Throughout the paper, a log structure on a scheme $X$ means a log structure on the étale site $X_{\text {et }}$ unless another topology is specified.

If $X$ is a log scheme we denote the underlying scheme by $X$, and if $f: X \rightarrow Y$ is a morphism of $\log$ schemes we denote the underlying morphism of schemes by $\stackrel{\circ}{f}: X \rightarrow \dot{Y}$. If $(\mathcal{M}, \alpha)$ is a log structure on a scheme $X$, we denote by $\lambda: \mathcal{O}_{X}^{*} \rightarrow \mathcal{M}$ the inverse of $\alpha^{-1}\left(\mathcal{O}_{X}^{*}\right) \rightarrow \mathcal{O}_{X}^{*}$, and by $\overline{\mathcal{M}}$ the sheaf $\mathcal{M} / \mathcal{O}_{X}^{*}$. If $X$ is a log scheme, we usually denote by $\left(\mathcal{M}_{X}, \alpha_{X}\right)$ (or simply $\left.\mathcal{M}_{X}\right)$ its $\log$ structure. If $\alpha: \mathcal{M} \rightarrow \mathcal{O}_{X}$ is a pre-log structure on a scheme $X$, then we denote by $\left(\mathcal{M}^{a}, \alpha\right)$ (or just $\mathcal{M}^{a}$ ) the associated log structure.

A monoid $P$ is called fine if it is finitely generated and integral, and is called $\mathrm{fs}$ if it is fine and saturated (i.e. if $a \in P^{g p}$ and there exists $r>0$ such that $r a \in P \subset P^{g p}$ then $a \in P$ ). We denote the set of invertible elements in $P$ by $P^{*}$. If $P$ is fine and $P^{*}=\{0\}$, then a nonzero element $p \in P$ is called irreducible if for every pair of elements $p_{1}, p_{2} \in P$ for which $p=p_{1}+p_{2}$ either $p_{1}$ or $p_{2}$ is zero.

Recall that a chart for a fine $\log$ structure $\mathcal{M}$ on a scheme $X$ is a map $P \rightarrow \mathcal{M}$ from the constant sheaf associated to a fine monoid $P$ such that $P^{a} \rightarrow \mathcal{M}$ is an isomorphism ([9], 2.9(1)). For a fine monoid $P$, we denote by $\operatorname{Spec}(P \rightarrow \mathbb{Z}[P])$ the log scheme with underlying scheme $\operatorname{Spec}(\mathbb{Z}[P])$ and $\log$ structure induced by the natural map $P \rightarrow \mathbb{Z}[P]$. If no confusion seems likely to arise, we may also abuse notation and write $\operatorname{Spec}(\mathbb{Z}[P])$ for the log scheme $\operatorname{Spec}(P \rightarrow \mathbb{Z}[P])$. Giving a chart $P \rightarrow \mathcal{M}$ for a $\log$ structure $\mathcal{M}$ on a scheme $X$ is equivalent to giving a morphism of $\log$ schemes

$$
\left(f, f^{b}\right):\left(X, \mathcal{M}_{X}\right) \rightarrow \operatorname{Spec}(P \rightarrow \mathbb{Z}[P])
$$

for which $f^{b}$ is an isomorphism (such a morphism is called strict ([8], 3.1)). If $S$ is a scheme, we write $S[P]$ for the scheme

$$
S[P]:=S \times_{\operatorname{Spec}(\mathbb{Z})} \operatorname{Spec}(\mathbb{Z}[P])
$$

and $\mathcal{M}_{S[P]}$ for the log structure on $S[P]$ induced by the log structure on $\operatorname{Spec}(\mathbb{Z}[P])$.

A chart for a morphism of fine log schemes $f: X \rightarrow S$ is a 5 -tuple $\left(Q, P, \beta_{Q}, \beta_{P}, \theta\right)$, where $\beta_{Q}: Q \rightarrow \mathcal{M}_{S}$ and $\beta_{P}: P \rightarrow \mathcal{M}_{X}$ are charts and $\theta: Q \rightarrow P$ is a morphism of fine monoids, such that the induced diagram of fine log schemes

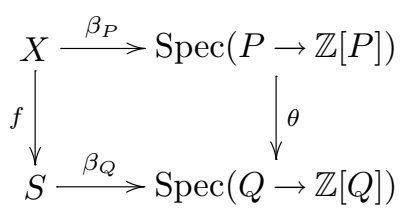

commutes ([9], 2.9(2)). If no confusion seems likely to arise, we sometimes write $(Q, P, \theta)$ for a chart $\left(Q, P, \beta_{Q}, \beta_{P}, \theta\right)$.

In general, given a morphism of fine monoids $\theta: Q \rightarrow P$ and a chart $\beta: Q \rightarrow \mathcal{M}$ for a fine $\log$ structure $\mathcal{M}$ on a scheme $X$, we let $X_{Q}[P]$ denote the scheme

$$
X_{Q}[P]:=X \times_{\operatorname{Spec}(\mathbb{Z}[Q])} \operatorname{Spec}(\mathbb{Z}[P]) .
$$

The natural map $P \rightarrow \mathcal{O}_{X_{Q}[P]}$ induces a $\log$ structure $\mathcal{M}_{X_{Q}[P]}$ on $X_{Q}[P]$, and there is a natural morphism of log schemes

$$
\left(X_{Q}[P], \mathcal{M}_{X_{Q}[P]}\right) \rightarrow(X, \mathcal{M})
$$


Regarding algebraic stacks we follow the conventions of [14], except we do not assume that our stacks are quasi-separated. More precisely, by an algebraic stack over a base scheme $S$, we mean a stack $\mathcal{X} / S$ in the sense of ([14], 3.1) satisfying the following:

(1.2.4) the diagonal

$$
\Delta: \mathcal{X} \rightarrow \mathcal{X} \times{ }_{S} \mathcal{X}
$$

is representable and of finite presentation;

(1.2.5) there exists a surjective smooth morphism $X \rightarrow \mathcal{X}$ from a scheme.

The reader is assumed to be familiar with algebraic stacks.

\section{Some remarks about charts}

In this section we prove two propositions about charts which will be used in the proof of (1.1), and we discuss two corollaries which will be used in Section 5.

Proposition 2.1. - Let $\mathcal{M}$ be a fine log structure on a scheme $X$ and let $x \in X$ be a point. Then there exist an fppf neighborhood $f: X^{\prime} \rightarrow X$ of $x$ and a chart $\beta: P \rightarrow f^{*} \mathcal{M}$ such that for some geometric point $\bar{x}^{\prime} \rightarrow X^{\prime}$ lying over $x$, the natural map $P \rightarrow f^{-1} \overline{\mathcal{M}}_{\bar{x}^{\prime}}$ is bijective. If $\overline{\mathcal{M}}_{\bar{x}}^{g p, t o r} \otimes k(x)=0$ (where $\overline{\mathcal{M}}_{\bar{x}}^{\text {gp tor }}$ denotes the torsion subgroup of $\overline{\mathcal{M}}_{\bar{x}}^{g p}$ ), then such a chart exists in an étale neighborhood of $x$.

Proof. - The key point is that the extension

$$
0 \rightarrow \mathcal{O}_{X, \bar{x}}^{*} \stackrel{\lambda}{\rightarrow} \mathcal{M}_{\bar{x}}^{g p} \rightarrow \overline{\mathcal{M}}_{\bar{x}}^{g p} \rightarrow 0
$$

splits after replacing $X$ by an fppf neighborhood of $x$. Indeed, choose an isomorphism $\overline{\mathcal{M}}_{\bar{x}}^{g p} \simeq$ $F \oplus G$, where $F$ is a free group and $G=\bigoplus_{i=1}^{m} G_{i}$ is a direct sum of finite cyclic groups $G_{i}$ generated by an element $g_{i} \in G_{i}$ of order $r_{i}$. Since $F$ is a finitely generated free abelian group, it is clear that the projection $\mathcal{M}_{\bar{x}}^{g p} \rightarrow F$ admits a section. In order to construct a section of the projection $\mathcal{M}_{\bar{x}}^{g p} \rightarrow \bigoplus G_{i}$, let $\tilde{g}_{i} \in \mathcal{M}_{\bar{x}}^{g p}$ be a lift of $g_{i}$. Then $r \tilde{g}_{i}$ (writing the group law additively) is equal to $\lambda\left(u_{i}\right)$ for some unit $u_{i} \in \mathcal{O}_{X, \bar{x}}^{*}$. Thus after replacing $\mathcal{O}_{X, \bar{x}}$ by the ring

$$
\mathcal{O}_{X, \bar{x}}\left[T_{1}, \ldots, T_{m}\right] /\left(T^{r_{i}}-u_{i}\right)_{i=1}^{m}
$$

and $\tilde{g}_{i}$ by $\tilde{g}_{i}-\lambda\left(T_{i}\right)$, the extension (2.1.1) splits. Note that (2.1.2) is a finite flat $\mathcal{O}_{X, \bar{x}}$-algebra and is an étale algebra if the $r_{i}$ are prime to $k(x)$; that is, if $\overline{\mathcal{M}}_{\bar{x}}^{g p, t o r} \otimes k(x)=0$.

Hence it suffices to prove the proposition under the additional hypothesis that the map $\mathcal{M}_{\bar{x}}^{g p} \rightarrow \overline{\mathcal{M}}_{\bar{x}}^{g p}$ admits a section $s: \overline{\mathcal{M}}_{\bar{x}}^{g p} \rightarrow \mathcal{M}_{\bar{x}}^{g p}$. Let $P:=\overline{\mathcal{M}}_{\bar{x}}$. Then since

$$
\mathcal{M}_{\bar{x}}=\mathcal{M}_{\bar{x}}^{g p} \times \overline{\mathcal{M}}_{\bar{x}}^{g p} \overline{\mathcal{M}}_{\bar{x}}
$$

(this follows from the definition of $\overline{\mathcal{M}}$ ), the map $s$ induces a map $\beta_{P}: P \rightarrow \mathcal{M}_{\bar{x}}$ such that the induced map $P \rightarrow \overline{\mathcal{M}}_{\bar{x}}$ is bijective. By $([9], 2.10)$ the map $\beta_{P}$ extends to a chart in some étale neighborhood of $\bar{x}$, and so the proposition follows.

Proposition 2.2.- Let $f: X \rightarrow S$ be a morphism of fine log schemes and suppose $\beta_{Q}: Q \rightarrow \mathcal{M}_{S}$ is a chart. Then étale locally on $X$ there exists a chart $\left(Q, P, \beta_{Q}, \beta_{P}, \theta\right)$ (with the same $\left.\left(Q, \beta_{Q}\right)\right)$ for $f$ with $\theta: Q \rightarrow P$ injective. If $f: X \rightarrow S$ is a morphism of $f$ s log schemes and if $Q$ is saturated and torsion free, then étale locally on ${ }_{X}^{\circ}$ there exists such a chart with $P$ saturated and torsion free. 
Proof. - Étale locally on $\stackrel{\circ}{X}$ we can find a chart $\beta_{P^{\prime}}: P^{\prime} \rightarrow \mathcal{M}_{X}$, and in the fs case, we can by (2.1) find a chart with $P^{\prime}$ fs and torsion free. Let $\bar{x} \rightarrow X$ be a geometric point, and define $P$ to be the fiber product of the diagram

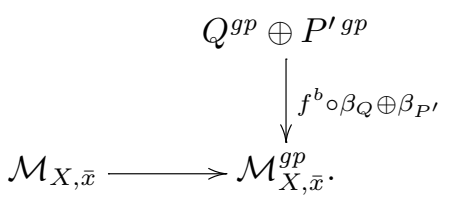

By ([9], 2.10), $P$ is a fine monoid, and in the fs case with $Q$ and $P^{\prime}$ saturated and torsion free, the monoid $P$ is again saturated and torsion free since it is a submonoid of $Q^{g p} \oplus P^{\prime g p}$ and $\mathcal{M}_{X, \bar{x}}$ is saturated. Let $\theta: Q \rightarrow P$ be the map induced by the map $Q \rightarrow Q^{g p} \oplus P^{\prime g p}$ which sends $q \in Q$ to $(q, 0)$, and let $\beta_{P}: P \rightarrow \mathcal{M}_{X, \bar{x}}$ denote the projection to $\mathcal{M}_{X, \bar{x}}$. After replacing $X$ by an étale neighborhood of $\bar{x}$, we can by $([9], 2.10)$ assume that $\beta_{P}$ extends to a global chart, which we also denote by $\beta_{P}$, for $\mathcal{M}_{X}$. Moreover, since $Q$ is finitely generated the resulting diagram

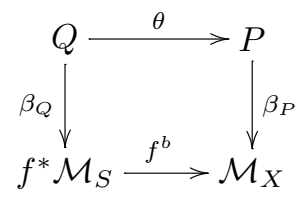

commutes in an étale neighborhood of $\bar{x}$, since it is commutative at $\bar{x}$ by construction. Thus $\left(Q, P, \beta_{Q}, \beta_{P}, \theta\right)$ defines a chart as desired in some étale neighborhood of $\bar{x}$.

In Section 5, we shall often consider the situation of a fine log structure $\mathcal{M}$ on a scheme $X$ and a map $P \rightarrow \overline{\mathcal{M}}$, where $P$ is a fine monoid. We therefore include the following two corollaries of (2.1):

COROLLARY 2.3. - Let $\mathcal{M}$ be a fine log structure on a scheme $X$, and suppose a morphism $\pi: P \rightarrow \overline{\mathcal{M}}$ from a fine monoid $P$ is given. Then in a fppf neighborhood of any point $x \in X$ there exists a lifting $\tilde{\pi}: P \rightarrow \mathcal{M}$ of $\pi$. If $\overline{\mathcal{M}}_{\bar{x}}^{g p, t o r} \otimes k(x)=0$, then a lifting $\tilde{\pi}$ of $\pi$ exists in an étale neighborhood of $x$.

Proof. - Let $r: \mathcal{M} \rightarrow \overline{\mathcal{M}}$ denote the quotient map. By (2.1), we can after replacing $X$ by a fppf neighborhood of $x$ assume that the map $r_{\bar{x}}: \mathcal{M}_{\bar{x}} \rightarrow \overline{\mathcal{M}}_{\bar{x}}$ admits a section (in the case when $\overline{\mathcal{M}}_{\bar{x}}^{g p, \text { tor }} \otimes k(x)=0$ it suffices by (2.1) to replace $X$ by an étale neighborhood of $x$ ). Hence we can find a map $\tilde{\pi}_{\bar{x}}: P \rightarrow \mathcal{M}_{\bar{x}}$ such that $r_{\bar{x}} \circ \tilde{\pi}_{\bar{x}}=\pi_{\bar{x}}$. Since $P$ is fine, we can extend the map $\tilde{\pi}_{\bar{x}}$ to a map $\tilde{\pi}: P \rightarrow \mathcal{M}$ in some étale neighborhood of $\bar{x}$. Now since $P$ is finitely generated, the two maps

$$
\pi, r \circ \tilde{\pi}: P \rightarrow \overline{\mathcal{M}}
$$

which are equal at $\bar{x}$, are equal in some étale neighborhood of $\bar{x}$. From this the result follows.

COROLlary 2.4. - Let $\mathcal{M}$ be a fine log structure on a scheme $X$, and suppose a map $\pi: P \rightarrow \overline{\mathcal{M}}$ from a fine monoid $P$ is given .

(i) If $\tilde{\pi}_{i}: P \rightarrow \mathcal{M}(i=1,2)$ are two lifts of $\pi$, then $\tilde{\pi}_{1}$ is a chart if and only if $\tilde{\pi}_{2}$ is a chart.

(ii) If $\mathcal{M}$ is $f s$, then $\pi$ lifts fppf locally on $X$ to a chart for $\mathcal{M}$ if and only if $\pi$ lifts étale locally to a chart for $\mathcal{M}$. 
Proof. - To see (i), note that for each $p \in P$ there exists a unique unit $u_{p} \in \mathcal{O}_{X}^{*}$ such that $\tilde{\pi}_{1}(p)=\lambda\left(u_{p}\right)+\tilde{\pi}_{2}(p)$. Hence if $\varepsilon_{i}: \mathcal{M}_{i} \rightarrow \mathcal{M}(i=1,2)$ denotes the morphism of fine $\log$ structures obtained from $\tilde{\pi}_{i}$, then the map

$$
P \rightarrow \mathcal{O}_{X}^{*} \oplus P, \quad p \mapsto\left(u_{p}, p\right)
$$

induces an isomorphism $\sigma: \mathcal{M}_{1} \rightarrow \mathcal{M}_{2}$ such that $\varepsilon_{1}=\varepsilon_{2} \circ \sigma$. Therefore, $\varepsilon_{1}$ is an isomorphism if and only if $\varepsilon_{2}$ is an isomorphism.

As for (ii), note first that the "if" direction is clear. To prove the "only if" direction suppose that there exists an fppf cover $f: X^{\prime} \rightarrow X$ such that $f^{-1}(\pi): P \rightarrow f^{-1} \overline{\mathcal{M}}$ lifts to a chart for $f^{*} \mathcal{M}$. By (2.3), we can after replacing $X$ by an étale cover assume that we have a lifting $\tilde{\pi}: P \rightarrow \mathcal{M}$ of $\pi$. We claim that the morphism of $\log$ structures $\varepsilon: \mathcal{M}^{\prime} \rightarrow \mathcal{M}$ obtained from $\tilde{\pi}$ is an isomorphism. To verify this, it suffices to show that the map $\bar{\varepsilon}: \overline{\mathcal{M}}^{\prime} \rightarrow \overline{\mathcal{M}}$ is an isomorphism ([8], 3.2), and since $f: X^{\prime} \rightarrow X$ is surjective it suffices to verify that the map $f^{-1}(\bar{\varepsilon}): f^{-1} \overline{\mathcal{M}}^{\prime} \rightarrow f^{-1} \overline{\mathcal{M}}$ is an isomorphism. But by (i), the map $f^{*}(\varepsilon): f^{*} \mathcal{M}^{\prime} \rightarrow \mathcal{M}$ is an isomorphism, since we are assuming that there exists some lift of $\pi$ over $X^{\prime}$ which is a chart. Hence the map $f^{-1}(\bar{\varepsilon})$ is also an isomorphism.

\section{Existence of universal log structures}

Fix a fine $\log$ scheme $S$ and let $\mathcal{L} o g_{S}$ be as defined in the introduction. In this section we prove Theorem (1.1) assuming the result that $\mathcal{L}_{0 g_{S}}$ is a stack with respect to the fppf topology (A.2).

Remark 3.1. - The fact that $\mathcal{L}_{\log }$ is a stack with respect to the fppf-topology is used in two places in the proof of (1.1):

(i) In the proof of (3.2), we use the fact that the functor $I$ defined in (3.4(i)) is a sheaf with respect to the fppf topology. This is because only a flat cover of $I$ is constructed (3.7), and in order to use ([14], 10.4.1) to prove that $I$ is an algebraic space we need that $I$ is a sheaf with respect to the fppf topology.

(ii) The cover of $\mathcal{L}_{o g}$ constructed in (3.16) is only a flat cover, and in order to apply ([14], 10.1) we need that $\mathcal{L}_{\log }$ is a stack with respect to the fppf topology.

One can show, however, that the substack $\mathcal{T}$ or $S \subset \mathcal{L}_{\text {og }}$ classifying fs log schemes over $S$ is an algebraic stack without using (A.2). In the case when the $\log$ structures $\mathcal{M}_{1}$ and $\mathcal{M}_{2}$ in (3.4(i)) are fs, the cover of $I$ constructed in (3.7) is in fact an étale cover (this follows from the proof). Moreover, the theory of toric stacks discussed in Section 5 yields a smooth cover of $\mathcal{T}$ or ${ }_{S}$ ((5.25)-(5.27)).

The proof of (1.1) will be in several steps ((3.2)-(3.16)). The reader may wish to consult examples (3.10), (3.11), and (3.18) before proceeding with the proof.

THEOREM 3.2. - The diagonal

$$
\Delta_{\mathcal{L} g_{S}}: \mathcal{L}_{o g} \rightarrow \mathcal{L} \operatorname{og}_{S} \times{ }_{S} \log _{S}
$$

is representable, locally separated, and of finite presentation.

Remark 3.3. - Recall ([12], II.3.9) that a morphism between algebraic spaces $f: I \rightarrow X$ is locally separated if the diagonal $I \rightarrow I \times_{X} I$ is a quasi-compact immersion. The statement that $f$ is locally separated can be verified étale locally on $X$ and is preserved under arbitrary base change $X^{\prime} \rightarrow X$. Hence by ([14], 3.10.1) it makes sense to say that a representable morphism of stacks is locally separated. 
Before giving the proof of (3.2) let us note the following corollary:

COROLLARY 3.4. - (i) Let

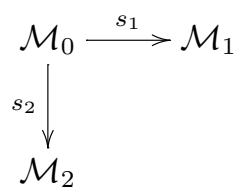

be a diagram of fine log structures on a scheme $X$. Then the functor I on $X$-schemes which to any $f: Z \rightarrow X$ associates the set of isomorphisms $\varepsilon: f^{*} \mathcal{M}_{1} \rightarrow f^{*} \mathcal{M}_{2}$ such that $\varepsilon \circ f^{*}\left(s_{1}\right)=f^{*}\left(s_{2}\right)$ is representable by a locally separated algebraic space of finite presentation over $X$.

(ii) Let $\mathcal{M}$ be a fine log structure on a scheme $X$. Then the functor $\underline{\operatorname{Aut}}(\mathcal{M})$ on $X$-schemes which to any $f: Z \rightarrow X$ associates the set of automorphisms of $f^{*} \overline{\mathcal{M}}$ is representable by a locally separated algebraic space of finite presentation over $X$.

Proof. - To see (i), note that the maps $s_{1}$ and $s_{2}$ define two morphisms of log schemes

$$
h_{1}:\left(X, \mathcal{M}_{1}\right) \rightarrow\left(X, \mathcal{M}_{0}\right), \quad h_{2}:\left(X, \mathcal{M}_{2}\right) \rightarrow\left(X, \mathcal{M}_{0}\right)
$$

and the functor $I$ is by definition the fiber product of the diagram

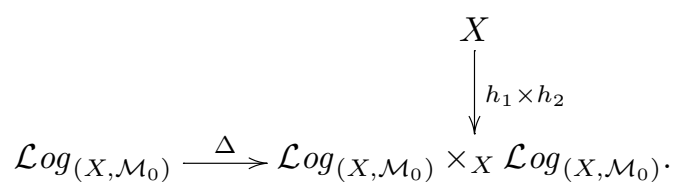

Hence (i) follows from (3.2).

Statement (ii) follows from (i) by taking $\mathcal{M}_{0}=\mathcal{O}_{X}^{*}$ and $\mathcal{M}_{1}=\mathcal{M}_{2}=\mathcal{M}$.

Proof of (3.2). - To say that the diagonal (3.2.1) is representable means that for any $\stackrel{\circ}{S}$-scheme $h: X \rightarrow \dot{S}$ and diagram of fine $\log$ structures on $X$

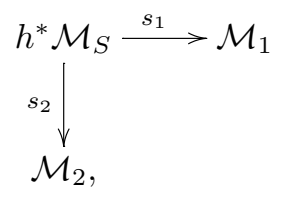

the functor $I$ on $X$-schemes obtained from (3.4(i)) by taking $\mathcal{M}_{0}=h^{*} \mathcal{M}_{S}$ is representable by a locally separated algebraic space of finite presentation over $X$.

Now to prove that $I$ is representable and locally separated we have to show that the diagonal $\Delta: I \rightarrow I \times_{X} I$ is representable by quasi-compact locally closed immersions, and that $I$ admits a flat cover of finite presentation over $X$ ([14], 10.4.1), since we know that $I$ is a sheaf with respect to the fppf topology by (A.2). The key tools will be (2.1) and the following lemma:

LEMMA 3.5. - Let $Y$ be a quasi-compact scheme and let $\mathcal{M}$ be a fine log structure on $Y$.

(i) Suppose given a chart $\beta: P \rightarrow \mathcal{M}$. For any geometric point $\bar{y} \rightarrow Y$ define

$$
F_{\bar{y}}:=\left\{p \in P \mid \alpha(p) \in \mathcal{O}_{Y, \bar{y}}^{*}\right\}
$$


and let $P_{F_{\bar{y}}} \subset P^{g p}$ be the submonoid generated by $P$ and $\left\{-f \mid f \in F_{\bar{y}}\right\}$. Then $P_{F_{\bar{y}}}^{*} \subset P^{g p}$ equals $F_{\bar{y}}^{g p}$ and the natural map

$$
P_{F_{\bar{y}}} / P_{F_{\bar{y}}}^{*} \rightarrow \overline{\mathcal{M}}_{\bar{y}}
$$

is an isomorphism.

(ii) The sheaf $\overline{\mathcal{M}}^{g p}$ is a constructible sheaf of $\mathbb{Z}$-modules ([3], IX.2.3).

(iii) If $\bar{\zeta} \rightarrow Y$ is a generization ([3], VIII.7.2) of a geometric point $\bar{y} \rightarrow Y$, then the specialization map

$$
\overline{\mathcal{M}}_{\bar{y}}^{g p} \rightarrow \overline{\mathcal{M}}_{\bar{\zeta}}^{g p}
$$

is surjective and identifies $\overline{\mathcal{M}}_{\bar{\zeta}}^{g p}$ with the quotient of $\overline{\mathcal{M}}_{\bar{y}}^{g p}$ by the subgroup generated by the image of

$$
\left\{m \in \mathcal{M}_{\bar{y}} \mid \alpha(m) \in \mathcal{O}_{Y, \bar{y}} \text { maps to a unit in } \mathcal{O}_{Y, \bar{\zeta}}\right\} .
$$

Proof. - To see the equality $P_{F_{\bar{y}}}^{*}=F_{\bar{y}}^{g p}$ in (i), note that clearly $F_{\bar{y}}^{g p} \subset P_{F_{\bar{y}}}^{*}$. On the other hand, if $p \in P_{F_{\bar{y}}}^{*}$, then we can write $p=p_{1}-f$ where $p_{1} \in P$ and $f \in F_{\bar{y}}$. Since the map $P \rightarrow \mathcal{M}_{\bar{y}}$ factors through $P_{F_{\bar{y}}}$, the image of $p_{1}$ in $\mathcal{M}_{\bar{y}}$ is a unit. Hence $p_{1} \in F_{\bar{y}}$ and $p \in F_{\bar{y}}^{g p}$.

From the equality $P_{F_{\bar{y}}}^{*}=F_{\bar{y}}^{g p}$ it follows that the map (3.5.3) is an isomorphism. Indeed the construction of the log structure associated to a pre-log structure ([9], 1.3) shows that (3.5.3) is surjective, and that the map $P \rightarrow \mathcal{M}_{\bar{y}}$ induces an isomorphism $\overline{\mathcal{M}}_{\bar{y}}^{g p} \simeq P^{g p} / F_{\bar{y}}^{g p}$.

To prove (ii), we may assume that we have a chart $P \rightarrow \Gamma(Y, \mathcal{M})$ from a fine monoid $P$, since the assertion is étale local on $Y$ by ([3], IX.2.4). We may also assume that $Y=\operatorname{Spec}(R)$ for some ring $R$. Let $p_{1}, \ldots, p_{n} \in P$ be elements which generate $P$, and for each subset $S \subset\{1, \ldots, n\}$ let

$$
U_{S}=\left(\bigcap_{i \in S} V\left(\alpha\left(p_{i}\right)\right)^{c}\right) \cap\left(\bigcap_{j \notin S} V\left(\alpha\left(p_{j}\right)\right)\right),
$$

where for $f \in R$ we denote by $V(f)$ the closed subscheme $\operatorname{Spec}(R /(f))$. Then $\bigcup U_{S}=Y$ and the $U_{S}$ are disjoint locally closed sub-schemes of $Y$. Moreover, the sheaf $\left.\overline{\mathcal{M}}^{g p}\right|_{U_{S}}$ is by (i) the constant sheaf associated to the quotient of $P^{g p}$ by the subgroup generated by $\left\{p_{i}\right\}_{i \in S}$.

To prove (iii), we may again assume that we have a chart $P \rightarrow \Gamma(Y, \mathcal{M})$. Then for any geometric point $\bar{y} \rightarrow Y$, the stalk $\overline{\mathcal{M}}_{\bar{y}}^{g p}$ is by (i) isomorphic to the quotient of $P^{g p}$ by the subgroup of $P^{g p}$ generated by the set of $p \in P$ for which $\alpha(p) \in \mathcal{O}_{Y, \bar{y}}^{*}$. From this the result follows.

With this we can begin the proof of Theorem (3.2). By replacing $S$ by $\left(X, h^{*} \mathcal{M}_{S}\right)$, we can assume that $\stackrel{\circ}{S}=X$.

\subsection{The diagonal $\Delta_{I}: I \rightarrow I \times_{X} I$ is representable by quasi-compact locally closed immersions}

Proof. - What has to be shown is that if $\sigma: \mathcal{M} \rightarrow \mathcal{M}$ is an automorphism of a fine log structure $\mathcal{M}$ on $X$, then the condition $\sigma=i d$ is represented by a quasi-compact locally closed immersion $Z \rightarrow X$.

Consider first the map $\bar{\sigma}: \overline{\mathcal{M}} \rightarrow \overline{\mathcal{M}}$. We claim that the condition $\bar{\sigma}=i d$ is represented by a quasi-compact open immersion $j: U \hookrightarrow X$. Indeed the set of points of $X$ where $\bar{\sigma}^{g p}=i d$ is constructible by (3.5(ii)) and stable under generization by (3.5(iii)). Hence the condition $\bar{\sigma}=i d$ is representable by an open immersion $j: U \hookrightarrow X$. To verify that $j$ is quasi-compact, we may replace $X$ by an étale cover and hence can assume that $X$ is affine and that $\mathcal{M}$ admit a global 
chart. In this case, the proof of (3.5(ii)) shows that there exists a finite stratification of $X$ by locally closed affine subschemes such that $\overline{\mathcal{M}}$ is constant on each stratum. Hence in this case $U$ is a finite union of affine schemes and hence is quasi-compact. Therefore, after replacing $X$ by an open set, we may assume that $\bar{\sigma}=i d$.

We claim that if $\bar{\sigma}=i d$, then the condition $\sigma=i d$ is represented by a closed subscheme of $X$. To see this, we may assume that we have a chart $\beta: P \rightarrow \mathcal{M}$. Since the map $\bar{\sigma}$ is equal to the identity, for each $p \in P$ there exists a unique unit $u_{p} \in \mathcal{O}_{X}^{*}$ such that

$$
\beta(p)=\sigma(\beta(p))+\lambda\left(u_{p}\right) .
$$

From this we conclude that if $\left\{p_{1}, \ldots, p_{r}\right\} \in P$ is a set of generators for $P$, then the condition $\sigma=i d$ is represented by the closed subscheme defined by the ideal

$$
\left(u_{1}-1, \ldots, u_{r}-1\right) .
$$

\section{7. $I$ admits a flat cover of finite presentation over $X$}

Proof. - For each point $x \in X$, we can by (2.1) find a fppf neighborhood $g: V \rightarrow X$ of $x$, a geometric point $\bar{x}^{\prime} \rightarrow V$ mapping to $x$, and charts $P_{i} \rightarrow g^{*} \mathcal{M}_{i}$ such that the maps $P_{i} \rightarrow \overline{\mathcal{M}}_{i, \bar{x}^{\prime}}$ are bijective $(i=1,2)$ (note that in the case when the $\mathcal{M}_{i}$ are saturated we can take $V$ to be an étale neighborhood of $x$ by (2.1)). Define fine monoids $Q_{i}$ by the formula

$$
Q_{i}:=P_{i}^{g p} \times_{g^{*} \mathcal{M}_{i, \bar{x}^{\prime}}^{g p}} g^{*} \mathcal{M}_{S, \bar{x}^{\prime}}
$$

and let $\beta_{i}: Q_{i} \rightarrow g^{*} \mathcal{M}_{S, \bar{x}^{\prime}}$ be the projection maps. By the definition of $Q_{i}$ the image of the composite

$$
Q_{i} \stackrel{\mathrm{pr}_{1}}{\rightarrow} P_{i}^{g p} \rightarrow \overline{\mathcal{M}}_{i, \bar{x}^{\prime}}^{g p}
$$

is contained in $\overline{\mathcal{M}}_{i, \bar{x}^{\prime}}$. Since $P_{i} \rightarrow \overline{\mathcal{M}}_{i, \bar{x}^{\prime}}$ is bijective it follows that the projection to $P_{i}^{g p}$ induces a map $\theta_{i}: Q_{i} \rightarrow P_{i}$.

Lemma 3.8. - The maps $\bar{\beta}_{i}: Q_{i} \rightarrow \overline{\mathcal{M}}_{S, \bar{x}^{\prime}}$ induced by the $\beta_{i}$ are bijective.

Proof. - If $m \in g^{*} \mathcal{M}_{S, \bar{x}^{\prime}}$, then there exists a unique unit $u(m)$ such that there exists $q \in Q_{i}$ with $\beta_{i}(q)=\lambda(u(m))+m$. Indeed since $P_{i} \rightarrow \overline{\mathcal{M}}_{i, \bar{x}^{\prime}}$ is bijective, there exists a unique unit $u(m)$ such that the image of $\lambda(u(m))+m$ in $g^{*} \mathcal{M}_{i, \bar{x}^{\prime}}$ is in the image of $P_{i}$. Thus the maps $\bar{\beta}_{i}: Q_{i} \rightarrow \overline{\mathcal{M}}_{S, \bar{x}^{\prime}}$ are surjective.

On the other hand, if $q_{j} \in Q_{i}(j=1,2)$ are two elements with $\bar{\beta}_{i}\left(q_{1}\right)=\bar{\beta}_{i}\left(q_{2}\right)$, then writing $q_{j}=\left(p_{j}, m_{j}\right)$ with $p_{j} \in P_{i}^{g p}$ and $m_{j} \in g^{*} \mathcal{M}_{S, \bar{x}^{\prime}}$, we see that there exists a unit $u$ such that $m_{1}=\lambda(u)+m_{2}$. Thus the images of $p_{1}$ and $p_{2}$ in $g^{*} \mathcal{M}_{i, \bar{x}^{\prime}}$ differ by a unit, and since the maps $P_{i} \rightarrow \overline{\mathcal{M}}_{\bar{x}^{\prime}}$ are bijective we must have $p_{1}=p_{2}$. Thus $m_{2}$ and $m_{2}+\lambda(u)$ have the same image in $g^{*} \mathcal{M}_{i, \bar{x}^{\prime}}$ and so $u=1$. From this it follows that $q_{1}=q_{2}$.

Put $Q=Q_{1}$. Since the maps $\bar{\beta}_{i}: Q_{i} \rightarrow \overline{\mathcal{M}}_{S, \bar{x}^{\prime}}$ are bijective, there exists a unique isomorphism $\varepsilon: Q \rightarrow Q_{2}$ such that $\bar{\beta}_{2} \circ \varepsilon=\bar{\beta}_{1}$. Hence for each $q \in Q$ there exists a unique unit $u_{q} \in \mathcal{O}_{V, \bar{x}^{\prime}}^{*}$ such that

$$
\beta_{1}(q)=\beta_{2}(\varepsilon(q))+\lambda\left(u_{q}\right),
$$

and the association $q \mapsto u_{q}$ defines a group homomorphism

$$
\rho: Q^{g p} \rightarrow \mathcal{O}_{V, \bar{x}^{\prime}}^{*}
$$


Let $G$ denote the image.

We then have a commutative diagram

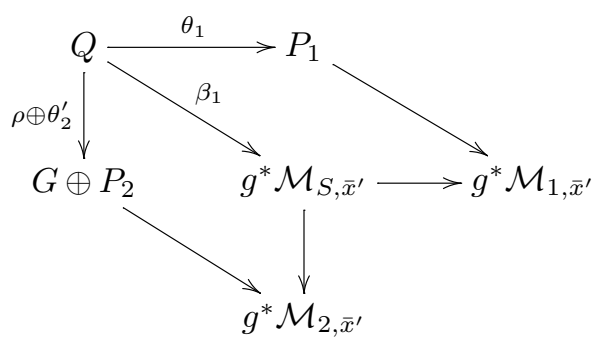

where $\theta_{2}^{\prime}:=\theta_{2} \circ \varepsilon$. Since our log structures are fine we can extend the data $\left(Q, P_{1}, \theta_{1}\right)$ and $\left(Q, G \oplus P_{2}, \rho \oplus \theta_{2}^{\prime}\right)$ to charts for the morphisms

$$
\left(V, g^{*} \mathcal{M}_{i}\right) \rightarrow\left(V, g^{*} \mathcal{M}_{S}\right)
$$

in some étale neighborhood of $\bar{x}^{\prime}$ using ([9], 2.10).

For each isomorphism $\sigma: P_{1} \rightarrow P_{2}$ for which $\theta_{2}^{\prime}=\sigma \circ \theta_{1}$, define a scheme

$$
C_{\sigma}:=\operatorname{Spec}_{V}\left(\mathcal{O}_{V}\left[P_{1}^{g p}\right] / J\right)
$$

where $J$ is the ideal sheaf generated by the equations

$$
\alpha_{2}(\sigma(p)) e(p)=\alpha_{1}(p), \quad p \in P_{1}
$$

and

$$
e\left(\theta_{1}(q)\right)=\rho(q), \quad q \in Q .
$$

Here $e(p)$ denotes the image of an element $p \in P_{1}$ in the group algebra $\mathcal{O}_{V}\left[P_{1}^{g p}\right]$, and we have abused notation and written $\alpha_{i}$ for the composite

$$
P_{i} \rightarrow g^{*} \mathcal{M}_{i} \rightarrow \mathcal{O}_{V}
$$

There is a tautological isomorphism $g^{*} \mathcal{M}_{1} \simeq g^{*} \mathcal{M}_{2}$ over $C_{\sigma}$ defined by the map

$$
p \mapsto \lambda(e(p))+\sigma(p), \quad p \in P_{1},
$$

which induces a morphism

$$
j:\left.C_{\sigma} \rightarrow I\right|_{V}
$$

We claim that $j$ makes $C_{\sigma}$ an open sub-functor of $\left.I\right|_{V}$.

To see this, observe that $C_{\sigma}$ represents the functor on $V$-schemes which to any $f: Z \rightarrow V$ associates the set of isomorphisms $\varepsilon:(g \circ f)^{*} \mathcal{M}_{1} \rightarrow(g \circ f)^{*} \mathcal{M}_{2}$ such that

$$
\varepsilon \circ(g \circ f)^{*}\left(s_{1}\right)=(g \circ f)^{*}\left(s_{2}\right),
$$


and for which the diagram

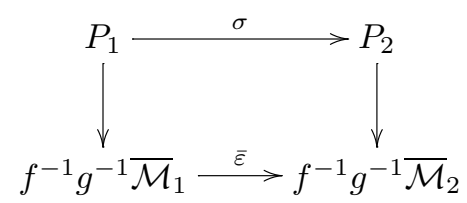

commutes. Indeed given such an $\varepsilon$, define a map to $\operatorname{Spec}\left(\mathcal{O}_{V}\left[P_{1}^{g p}\right]\right)$ by sending an element $e(p)$ $\left(p \in P_{1}\right.$ ) to the unique unit $u_{p} \in \mathcal{O}_{Z}^{*}$ for which

$$
\varepsilon(p)=\lambda\left(u_{p}\right)+\sigma(p) .
$$

This map to $\operatorname{Spec}\left(\mathcal{O}_{V}\left[P_{1}^{g p}\right]\right)$ factors through $C_{\sigma}$ : the equations in (3.8.2) are satisfied because $\alpha_{2} \circ \varepsilon=\alpha_{1}$, and the equations in (3.8.3) are satisfied because $\varepsilon \circ(g \circ f)^{*}\left(s_{1}\right)=(g \circ f)^{*}\left(s_{2}\right)$.

By (3.5), the condition that (3.8.4) commutes is an open condition, and hence $C_{\sigma}$ is an open sub-functor of $\left.I\right|_{V}$. In particular, the map $C_{\sigma} \rightarrow I$ is flat.

The union of all $C_{\sigma}$ constructed in the manner above cover $I$. Indeed given a map $\varepsilon: Z \rightarrow I$ and a point $z \in Z$ mapping to some $x \in X$, we can, by the argument given above, fppf locally find charts $\left(Q, P_{1}, \theta_{1}\right)$ and $\left(Q, G \oplus P_{2}, \rho \oplus \theta_{2}^{\prime}\right)$ for the morphisms

$$
\left(X, \mathcal{M}_{i}\right) \rightarrow\left(X, \mathcal{M}_{S}\right)
$$

as in (3.8.1), such that the maps

$$
P_{i} \rightarrow \overline{\mathcal{M}}_{i, \bar{x}}
$$

are bijective.

The isomorphism $\varepsilon$ hence induces an isomorphism $\sigma: P_{1} \simeq P_{2}$ such that $\theta_{2}^{\prime}=\sigma \circ \theta_{1}$ and such that diagram (3.8.4) over $Z$ commutes. Therefore by the functorial description of $C_{\sigma}$, the image of the map

$$
C_{\sigma} \times_{I} Z \rightarrow Z
$$

contains $z$.

Finally to see that $I \rightarrow X$ is quasi-compact, we may assume that $X$ is affine. In addition, we may replace $X$ by an étale cover so we may assume that all log structures involved admit global charts. In this case, there exists by the proof of (3.5) a finite stratification $\left\{X_{i}\right\}$ by locally closed affine subschemes of $X$ over which the sheaves $\overline{\mathcal{M}}_{S}, \overline{\mathcal{M}}_{1}$, and $\overline{\mathcal{M}}_{2}$ are all constant. By base changing to the $X_{i}$ we can therefore assume that these sheaves are constant over $X$. Replacing $X$ by another cover we can also assume that we have a diagram of charts as in (3.8.1) such that the maps $P_{i} \rightarrow \overline{\mathcal{M}}_{i}$ are isomorphisms. In this case $I$ is representable by the disjoint union over the set of isomorphisms $\sigma: P_{1} \simeq P_{2}$ satisfying $\theta_{2}^{\prime}=\sigma \circ \theta_{1}$ of the $C_{\sigma}$. As shown in the following lemma (3.9), the set of such isomorphisms is finite, and hence $I$ is quasi-compact.

LEMma 3.9. - Let $P$ be a fine monoid with $P^{*}=\{0\}$.

(i) The set $\operatorname{Irr}(P)$ of irreducible elements in $P$ is finite and generates $P$.

(ii) The automorphism group $\operatorname{Aut}(P)$ of $P$ is finite.

Proof. - To see (i), let $\left\{p_{1}, \ldots, p_{n}\right\}$ be a set of generators for $P$ with $n$ minimal. If $p \in \operatorname{Irr}(P)$, then $p$ must be one of the $p_{i}$, for if we write $p=\sum_{i} a_{i} p_{i}$ then the irreducibility of $p$ implies that $\sum_{i} a_{i}=1$. Hence $\operatorname{Irr}(P)$ is finite and there is an inclusion $\operatorname{Irr}(P) \subset\left\{p_{1}, \ldots, p_{n}\right\}$ which we 
claim is a bijection. Indeed, suppose one of the $p_{i}$, say $p_{n}$, is not irreducible. Then $p_{n}=p+q$ for some non-zero $p, q \in P$. The elements $p$ and $q$ must be in the submonoid of $P$ generated by $\left\{p_{1}, \ldots, p_{n-1}\right\}$, for otherwise we can write $p=p^{\prime}+p_{n}$ for some $p^{\prime} \in P$ (after possibly interchanging $p$ and $q$ ) which implies that $q$ is a unit; a contradiction. But if $p$ and $q$ are in the submonoid generated by $\left\{p_{1}, \ldots, p_{n-1}\right\}$, then $P$ is also generated by this set contradicting the minimality of our set of generators. Hence $P$ is generated by $\operatorname{Irr}(P)$.

Statement (ii) follows from (i), because any automorphism of $P$ must map $\operatorname{Irr}(P)$ to itself, and since this finite set generates $P$ we obtain an inclusion $\operatorname{Aut}(P) \subset \operatorname{Aut}(\operatorname{Irr}(P))$.

This concludes the proof of (3.2).

Example 3.10. - Let $X=\mathbb{A}^{1}=\operatorname{Spec}(\mathbb{Z}[T])$ be the affine line with log structure $\mathcal{M}_{X}$ associated to the map $\mathbb{N} \rightarrow \mathbb{Z}[T], 1 \mapsto T$. Then the algebraic space $\underline{\operatorname{Aut}}\left(\mathcal{M}_{X}\right)$ can be described as follows. Since a free monoid of rank 0 or 1 has no automorphisms, any automorphism $\varepsilon: \mathcal{M}_{X} \rightarrow \mathcal{M}_{X}$ must induce the identity map on $\overline{\mathcal{M}}_{X}$. Thus if $\varepsilon \in \underline{\operatorname{Aut}}\left(\mathcal{M}_{X}\right)(Y)$ for some $f: Y \rightarrow X, \varepsilon$ is induced by a map

$$
\mathbb{N} \rightarrow \mathcal{O}_{Y}^{*} \oplus \mathbb{N}, \quad 1 \mapsto(u, 1),
$$

where $u$ is a unit satisfying $u T=T$. Conversely, any such unit $u$ gives rise to an automorphism of $f^{*} \mathcal{M}_{X}$ by the same formula (3.10.1), and so there is an isomorphism

$$
\underline{\operatorname{Aut}}\left(\mathcal{M}_{X}\right) \simeq \operatorname{Spec}\left(\mathcal{O}_{X}\left[U^{ \pm}\right] / T(U-1)\right)
$$

Example 3.11. - More generally, let us construct directly the fiber product $I$ of the diagram

$$
\begin{aligned}
X & =\operatorname{Spec}\left(\mathbb{Z}\left[X_{1}, \ldots, X_{r}\right]\right) \\
Y=\operatorname{Spec}\left(\mathbb{Z}\left[Y_{1}, \ldots, Y_{r}\right]\right) \stackrel{\mathcal{M}_{Y}}{\longrightarrow} & \mathcal{L}_{X} \operatorname{Mog}_{\left(\operatorname{Spec}(\mathbb{Z}), \mathcal{O}_{\text {Spec }(\mathbb{Z})}^{*}\right)},
\end{aligned}
$$

where $r \geqslant 1$ is an integer and $\mathcal{M}_{X}$ (resp. $\mathcal{M}_{Y}$ ) denotes the log structure associated to

$$
\mathbb{N}^{r} \rightarrow \mathcal{O}_{X}, \quad\left(a_{i}\right)_{i=1}^{r} \mapsto \prod_{i=1}^{r} X_{i}^{a_{i}} \quad\left(\operatorname{resp} . \mathbb{N}^{r} \rightarrow \mathcal{O}_{Y},\left(a_{i}\right)_{i=1}^{r} \mapsto \prod_{i=1}^{r} Y_{i}^{a_{i}}\right)
$$

Let $S_{r}$ denote the symmetric group on $r$ letters, and for every $\sigma \in S_{r}$ let $I_{\sigma}$ be the $X \times Y$-scheme whose underlying scheme is $X \times \mathbb{G}_{m}^{r}$ and whose structure morphism is induced by the projection to $X$ and the map $\rho_{\sigma}$ to $Y$ given by

$$
\rho_{\sigma}^{*}: \mathbb{Z}\left[Y_{i}\right]_{i=1}^{r} \rightarrow \mathbb{Z}\left[X_{i}, U_{i}^{ \pm}\right]_{i=1}^{r}, \quad Y_{\sigma(i)} \mapsto U_{i} X_{i}
$$

Let $\widetilde{I}:=\coprod_{\sigma \in S_{r}} I_{\sigma}, \operatorname{pr}: \widetilde{I} \rightarrow X$ the projection, and $\rho:=\coprod \rho_{\sigma}: \widetilde{I} \rightarrow Y$ the map obtained from the $\rho_{\sigma}$.

Over $I_{\sigma}$ there is a natural isomorphism $\iota_{\sigma}: \operatorname{pr}^{*} \mathcal{M}_{X} \rightarrow \rho_{\sigma}^{*} \mathcal{M}_{Y}$ induced by the map

$$
\mathbb{N}^{r} \rightarrow \mathcal{O}_{X \times \mathbb{G}_{m}^{r}}^{*} \oplus \mathbb{N}^{r}, \quad e_{i} \mapsto\left(U_{i}^{-1}, e_{\sigma(i)}\right)
$$


Thus there is a natural map $\pi: \widetilde{I} \rightarrow I$. Note also that over $I_{\sigma}$ there is a natural commutative diagram

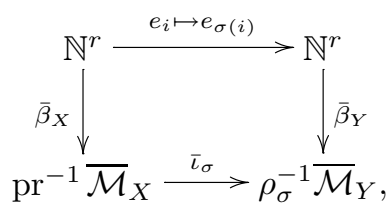

where $\beta_{X}$ (resp. $\beta_{Y}$ ) denotes the chart $\mathbb{N}^{r} \rightarrow \mathcal{M}_{X}$ (resp. $\mathbb{N}^{r} \rightarrow \mathcal{M}_{Y}$ ) used in the construction of $\mathcal{M}_{X}\left(\operatorname{resp} . \mathcal{M}_{Y}\right)$.

In fact, $\widetilde{I}$ represents the functor which to any $g_{1} \times g_{2}: Z \rightarrow X \times Y$ associates the set of pairs $(\iota, \sigma)$, where $\iota: g_{1}^{*} \mathcal{M}_{X} \rightarrow g_{2}^{*} \mathcal{M}_{Y}$ is an isomorphism and $\sigma \in S_{r}$ is a permutation such that the diagram

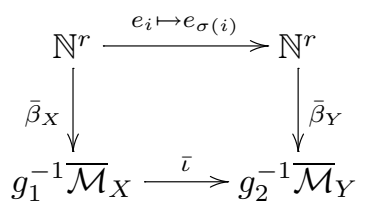

commutes. Indeed given such a pair $(\iota, \sigma)$ over some $X$-scheme $f: Z \rightarrow X$, define a map $h: Z \rightarrow I_{\sigma} \simeq X \times \mathbb{G}_{m}^{r}$ over $X$ by

$$
\mathcal{O}_{\mathbb{G}_{m}^{r}} \simeq \mathbb{Z}\left[U_{1}^{ \pm}, \ldots, U_{r}^{ \pm}\right] \rightarrow \Gamma\left(Z, \mathcal{O}_{Z}\right), \quad U_{i} \mapsto u_{i},
$$

where $u_{i} \in \Gamma\left(Z, \mathcal{O}_{Z}^{*}\right)$ denotes the unique unit such that $\iota\left(\beta_{X}\left(e_{i}\right)\right)=-\lambda\left(u_{i}\right)+\beta_{Y}\left(e_{\sigma(i)}\right)$ (such a unit exists by the commutativity of (3.11.3)). Then $\iota=h^{*}\left(\iota_{\sigma}\right)$ and $h$ is the unique map with this property.

Next observe that for any morphism

$$
g_{1} \times g_{2}: Z \rightarrow X \times Y
$$

and isomorphism

$$
\iota: g_{1}^{*} \mathcal{M}_{X} \simeq g_{2}^{*} \mathcal{M}_{Y}
$$

there exists étale locally on $Z$ an element $\sigma \in S_{r}$ such that diagram (3.11.3) commutes. To verify this we may assume that $Z$ is the spectrum of a strictly henselian local ring. Let $z \in Z$ be the closed point. Then the maps

$$
\bar{\beta}_{X}: \mathbb{N}^{r} \rightarrow\left(g_{1}^{-1} \overline{\mathcal{M}}_{X}\right)_{z}, \quad \bar{\beta}_{Y}: \mathbb{N}^{r} \rightarrow\left(g_{2}^{-1} \overline{\mathcal{M}}_{Y}\right)_{z}
$$

admit sections by (3.5(i)) giving isomorphisms

$$
\mathbb{N}^{r} \simeq \mathbb{N}^{r^{\prime}} \oplus\left(g_{1}^{-1} \overline{\mathcal{M}}_{X}\right)_{z} \quad \text { and } \quad \mathbb{N}^{r} \simeq \mathbb{N}^{r^{\prime}} \oplus\left(g_{2}^{-1} \overline{\mathcal{M}}_{Y}\right)_{z}
$$

for some $r^{\prime} \leqslant r$. Extending the isomorphism $\bar{\iota}_{z}$ in some way to all of $\mathbb{N}^{r}$ we obtain a permutation $\sigma \in S_{r}$ such that diagram (3.11.3) commutes at $z \in Z$. But then by (3.5(iii)) the diagram (3.11.3) commutes everywhere since $Z$ is the spectrum of a strictly henselian local ring, and $\sigma$ is the desired permutation.

It follows that $I$ is the quotient of $\widetilde{I}$ by the equivalence relation defined by the subfunctor $\Gamma \subset \widetilde{I} \times_{X \times Y} \widetilde{I}$ consisting of pairs $\left\{(\iota, \sigma),\left(\iota, \sigma^{\prime}\right)\right\}$. To show that $I$ is representable by an 
algebraic space it suffices to show that $\Gamma$ is representable and that the two projections $\Gamma \rightrightarrows \widetilde{I}$ are étale. Let $\Gamma_{\sigma, \sigma^{\prime}} \subset \Gamma$ be the fiber product of functors $\left.\Gamma \times_{(\widetilde{I} \times X \times Y} \widetilde{I}\right)\left(I_{\sigma} \times_{X \times Y} I_{\sigma^{\prime}}\right)$ so that $\Gamma=\coprod_{\sigma, \sigma^{\prime}} \Gamma_{\sigma, \sigma^{\prime}}$. The functor $\Gamma_{\sigma, \sigma^{\prime}}$ associates to any $g_{1} \times g_{2}: Z \rightarrow X \times Y$ the set of isomorphisms $\iota: g_{1}^{*} \mathcal{M}_{X} \simeq g_{2}^{*} \mathcal{M}_{Y}$ for which the diagram (3.11.3) commutes for both $\sigma$ and $\sigma^{\prime}$. Thus the first (resp. second) projection identifies $\Gamma_{\sigma, \sigma^{\prime}}$ with a subfunctor of $I_{\sigma}$ (resp. $I_{\sigma^{\prime}}$ ). Moreover, the condition that diagram (3.11.3) commutes with both $\sigma$ and $\sigma^{\prime}$ is an open condition by lemma (3.5(ii)) and (3.5(iii)). Therefore, $\Gamma_{\sigma, \sigma^{\prime}}$ maps isomorphically onto open subschemes of $I_{\sigma}$ and $I_{\sigma^{\prime}}$. In particular, $\Gamma$ is representable and the two projections to $\widetilde{I}$ are étale.

Remark 3.12. - The functor $I$ of (3.4(i)) is not separated in general. For an explicit example where the valuative criterion for separation fails, let $A$ be a discrete valuation ring with uniformizer $\pi$, and let $\mathcal{M}$ be the $\log$ structure associated to the chart

$$
\mathbb{N} \oplus \mathbb{N} \rightarrow A, \quad(i, j) \mapsto \pi^{i+j} .
$$

Then the identity map and the map induced by

$$
(i, j) \mapsto(j, i)
$$

are two automorphisms of $\mathcal{M}$ both of which induce the identity map on the generic fiber.

THEOREM 3.13. - Let $Q$ be a fine monoid and let

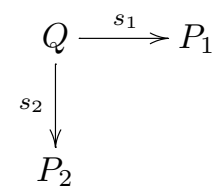

be a diagram of fine monoids with $s_{1}$ and $s_{2}$ injective. Define

$$
S=\operatorname{Spec}(Q \rightarrow \mathbb{Z}[Q]), \quad X=\operatorname{Spec}\left(P_{1} \rightarrow \mathbb{Z}\left[P_{1}\right]\right), \quad Y=\operatorname{Spec}\left(P_{2} \rightarrow \mathbb{Z}\left[P_{2}\right]\right),
$$

and let $h: \stackrel{\circ}{X} \times{ }_{S} \stackrel{\circ}{Y} \rightarrow \stackrel{\circ}{S}$ be the natural map. Denote by $I$ the algebraic space over $\stackrel{\circ}{X} \times_{S} \stackrel{\circ}{Y}$ obtained from (3.4(i)) applied to the diagram

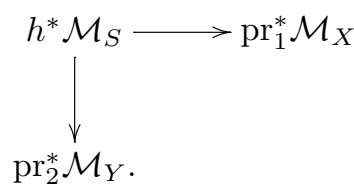

Then the two projections

$$
I \rightarrow \stackrel{\circ}{X}, \quad I \rightarrow \stackrel{\circ}{Y}
$$

are flat.

Proof. - For any fine monoid $P$ let $P^{*} \subset P$ be the set of invertible elements and let $\bar{P}=P / P^{*}$. Denote by $r(P)$ the minimal number of generators of $P$. If $P^{*}=\{0\}$, then by (3.9(i)) the number $r(P)$ is equal to the number of irreducible elements in $P$. 
LEMMA 3.14. - Let $X$ be a scheme, and let $P \rightarrow \mathcal{M}$ be a chart for a fine log structure $\mathcal{M}$ on $X$.

(i) Let $\bar{x} \rightarrow X$ be a geometric point and let $F_{\bar{x}} \subset P^{g p}$ be the submonoid defined in (3.5(i)). Then $F_{\bar{x}}$ is finitely generated and if $F_{\bar{x}} \neq P^{*}$ then $r\left(P_{F_{\bar{x}}} / P_{F_{\bar{x}}}^{*}\right)<r(\bar{P})$.

(ii) If $\bar{x} \rightarrow X$ is a geometric point with $r\left(\overline{\mathcal{M}}_{\bar{x}}\right)=r(\bar{P})$, then the map $\bar{P} \rightarrow \overline{\mathcal{M}}_{\bar{x}}$ is an isomorphism.

Proof. - To prove (i), let $p_{1}, \ldots, p_{r(\bar{P})} \in P$ be a set of elements whose images generate $\bar{P}$. Then $F_{\bar{x}}$ is the submonoid generated by $P^{*}$ and those $p_{i}$ for which $\alpha\left(p_{i}\right) \in \mathcal{O}_{X, \bar{x}}^{*}$. Indeed, any $p \in F_{\bar{x}}$ can be written as

$$
p=u+\sum n_{i} p_{i}, \quad u \in P^{*}, n_{i} \in \mathbb{N},
$$

and if $\alpha(p)=\alpha(u) \prod_{i} \alpha\left(p_{i}\right)^{n_{i}}$ is a unit in $\mathcal{O}_{X, \bar{x}}$, then for all $i$ with $n_{i}>0$ we must have $\alpha\left(p_{i}\right) \in \mathcal{O}_{X, \bar{x}}^{*}$. Hence $F_{\bar{x}}$ is finitely generated. Moreover, if $F_{\bar{x}} \neq P^{*}$ then at least one $p_{i}$ maps to a unit in $\mathcal{O}_{X, \bar{x}}$ which implies that $r\left(P_{F_{\bar{x}}} / P_{F_{\bar{x}}}^{*}\right)<r(\bar{P})$.

Statement (ii) follows from (3.5(i)) and (i).

To prove Theorem (3.13), it suffices to prove that the map $I \rightarrow \stackrel{\circ}{X}$ is flat. We proceed by induction on $n:=r\left(\bar{P}_{1}\right)$.

If $n=0$, then $P_{1}=P_{1}^{*}$ and so $\mathcal{M}_{X}$ is trivial. Hence if $g_{1} \times g_{2}: Z \rightarrow \stackrel{\circ}{X} \times \stackrel{\circ}{Y}$ is a morphism, there exists an isomorphism $g_{1}^{*} \operatorname{pr}_{1}^{*} \mathcal{M}_{X} \simeq g_{2}^{*} \operatorname{pr}_{2}^{*} \mathcal{M}_{Y}$ if and only if $g_{2}^{*} \operatorname{pr}_{2}^{*} \mathcal{M}_{Y}$ is trivial. Therefore,

$$
I \simeq \operatorname{Spec}\left(\mathbb{Z}\left[P_{1}^{*}\right]\right) \times_{\operatorname{Spec}\left(\mathbb{Z}\left[Q^{g p}\right]\right)} \operatorname{Spec}\left(\mathbb{Z}\left[P_{2}^{g p}\right]\right) .
$$

Now $\mathbb{Z}\left[P_{2}^{g p}\right] / \mathbb{Z}\left[Q^{g p}\right]$ is flat since $Q^{g p} \rightarrow P_{2}^{g p}$ is injective, and hence $I / \mathbb{Z}\left[P_{1}^{*}\right]$ is also flat.

Next we prove the theorem for $n$ assuming the result for $n-1$. If $x \in X$ is a point with $r\left(\overline{\mathcal{M}}_{X, \bar{x}}\right)<n$, then $x$ lies in the open set

$$
\operatorname{Spec}\left(\mathbb{Z}\left[P_{1, F_{\bar{x}}}\right]\right) \hookrightarrow \operatorname{Spec}\left(\mathbb{Z}\left[P_{1}\right]\right),
$$

and $r\left(\overline{\mathcal{M}}_{X, \bar{x}^{\prime}}\right)<n$ for any other point $x^{\prime} \in \operatorname{Spec}\left(\mathbb{Z}\left[P_{1, F_{\bar{x}}}\right]\right)$ by (3.5(i)) and (3.14(i)). Moreover, by induction the theorem holds over $\operatorname{Spec}\left(\mathbb{Z}\left[P_{1, F_{\bar{x}}}\right]\right)$. It follows that the set of points $x \in \stackrel{\circ}{X}$ with $r\left(\overline{\mathcal{M}}_{X, \bar{x}}\right)<n$ is an open set $U^{<n}$, and that the theorem holds over $U^{<n}$.

Combining this with Lemma (3.14(ii)), we see that it suffices to show that $I \rightarrow X$ is flat over points $x \in \stackrel{\circ}{X}$ where the map $\bar{P}_{1} \rightarrow \overline{\mathcal{M}}_{X, \bar{x}}$ is bijective.

We can assume that $P_{1} \simeq P_{1}^{*} \oplus \bar{P}_{1}$ by replacing $P_{1}$ by $G \oplus_{P_{1}^{*}} P_{1}$ for a suitable finitely generated abelian group $G$. Indeed we can choose an inclusion $P_{1}^{*} \hookrightarrow G$ such that the pushout of the sequence

$$
0 \rightarrow P_{1}^{*} \rightarrow P_{1}^{g p} \rightarrow \bar{P}_{1}^{g p} \rightarrow 0
$$

splits. Choose one such isomorphism $G \oplus_{P_{1}^{*}} P_{1}^{g p} \simeq G \oplus \bar{P}_{1}^{g p}$. By the universal property of pushout and the group associated to a monoid, the natural map

$$
\left(G \oplus_{P_{1}^{*}} P_{1}\right)^{g p} \rightarrow G \oplus_{P_{1}^{*}} P_{1}^{g p}
$$

is an isomorphism, and so the composite

$$
G \oplus_{P_{1}^{*}} P_{1} \rightarrow G \oplus_{P_{1}^{*}} P_{1}^{g p} \simeq G \oplus \bar{P}_{1}^{g p}
$$


is injective. Moreover, the image of (3.14.1) is equal to $G \oplus \bar{P}_{1}$, and so there exists a splitting $G \oplus_{P_{1}^{*}} P_{1} \simeq G \oplus \bar{P}_{1}$.

The resulting map

$$
\mathbb{Z}\left[P_{1}\right] \rightarrow \mathbb{Z}\left[G \oplus_{P_{1}^{*}} P_{1}\right] \simeq \mathbb{Z}[G] \otimes_{\mathbb{Z}\left[P_{1}^{*}\right]} \mathbb{Z}\left[P_{1}\right]
$$

is faithfully flat, and since verification of the flatness of $I \rightarrow \AA^{\circ}$ can be done after replacing $\AA^{\circ}$ by a flat cover, we may replace $P_{1}$ by $G \oplus P_{1}^{*} P_{1}$.

We can also assume that $\bar{P}_{2}$ is generated by $n$ elements and that we have a splitting $P_{2} \simeq P_{2}^{*} \oplus \overline{P_{2}}$. To see this, observe that the image of $I$ is contained in the union of the open subsets

$$
\stackrel{\circ}{X} \times_{S} \operatorname{Spec}\left(\mathbb{Z}\left[P_{2, F_{\bar{y}}}\right]\right) \subset \stackrel{\circ}{X} \times_{\dot{S}} \stackrel{\circ}{Y}
$$

where $\bar{y} \rightarrow \stackrel{\circ}{Y}$ is a geometric point with $r\left(\overline{\mathcal{M}}_{2, \bar{y}}\right) \leqslant n$. Hence we may replace $P_{2}$ by $P_{2, F_{\bar{y}}}$. In addition, we may assume that $P_{2} \simeq P_{2}^{*} \oplus \bar{P}_{2}$ by replacing $Y$ by

$$
Y^{\prime}=\operatorname{Spec}\left(G \oplus_{P_{2}^{*}} P_{2} \rightarrow \mathbb{Z}\left[G \oplus_{P_{2}^{*}} P_{2}\right]\right)
$$

for a suitable group $G$ as discussed above for $P_{1}$. Then, if $I^{\prime}$ denotes the algebraic space obtained from $X$ and $Y^{\prime}$, we have a flat surjection

$$
I^{\prime} \rightarrow I
$$

and since the property of being flat is fppf-local on domain it suffices to consider $I^{\prime}$.

Fix splittings $P_{1} \simeq P_{1}^{*} \oplus \bar{P}_{1}$ and $P_{2} \simeq P_{2}^{*} \oplus \bar{P}_{2}$, and let

$$
\rho_{i}: Q^{g p} \rightarrow P_{i}^{*}, \quad \theta_{i}: Q \rightarrow \bar{P}_{i}, \quad i=1,2
$$

be the maps induced by the composites

$$
Q \rightarrow P_{i} \simeq P_{i}^{*} \oplus \bar{P}_{i} \overbrace{\bar{P}_{i} .}^{P_{i}^{*}}
$$

If $\beta_{Q}: Q \rightarrow \mathcal{M}_{S}$ (resp. $\beta_{1}: P_{1} \rightarrow \mathcal{M}_{X}, \beta_{2}: P_{2} \rightarrow \mathcal{M}_{Y}$ ) denotes the natural chart, then viewing $\beta_{i}$ as a map from $P_{i}^{*} \oplus \bar{P}_{i}$ using the isomorphisms $P_{i} \simeq P_{i}^{*} \oplus \bar{P}_{i}$ we obtain charts $\left(Q, P_{i}^{*} \oplus \bar{P}_{i}, \beta_{Q}, \beta_{i}, \rho_{i} \oplus \theta_{i}\right)$ for the morphisms $X \rightarrow S$ and $Y \rightarrow S$.

For each isomorphism $\sigma: \bar{P}_{1} \rightarrow \bar{P}_{2}$ for which $\sigma \circ \theta_{1}=\theta_{2}$, define a scheme

$$
C_{\sigma}:=\operatorname{Spec}_{\dot{X} \times \times_{\dot{S}} \dot{Y}}\left(\mathcal{O}_{\dot{X} \times{ }_{S} \dot{Y}}\left[\bar{P}_{1}^{g p}\right] / J\right),
$$

where $J$ is the ideal sheaf generated by the equations

$$
\alpha_{2}(\sigma(p)) e(p)=\alpha_{1}(p), \quad p \in \bar{P}_{1}
$$

and

$$
e\left(\theta_{1}(q)\right)=\alpha_{2}\left(\rho_{2}(q)\right) \alpha_{1}\left(\rho_{1}(q)\right)^{-1}, \quad q \in Q .
$$


Then just as in the proof of (3.2), there is a natural open immersion $C_{\sigma} \rightarrow I$, and the union of the $C_{\sigma}$ cover the set of points of $I$ lying over points $x \in \stackrel{\circ}{X}$ for which $\bar{P}_{1} \rightarrow \overline{\mathcal{M}}_{X, \bar{x}}$ is bijective. Therefore it suffices to show that $\mathcal{O}_{C_{\sigma}}$ is flat over $\mathbb{Z}\left[P_{1}\right]$.

This follows from the definitions. Writing out the definition of $C_{\sigma}$ one finds that

$$
\mathcal{O}_{C_{\sigma}} \simeq \mathbb{Z}\left[\bar{P}_{1}\right]\left[P_{1}^{*} \oplus_{Q^{g p}} P_{2}^{g p}\right]
$$

and hence it suffices to show that

$$
\mathbb{Z}\left[P_{1}^{*}\right] \rightarrow \mathbb{Z}\left[P_{1}^{*} \oplus_{Q^{g p}} P_{2}^{g p}\right]
$$

is flat. This follows from the fact that $Q^{g p} \rightarrow P_{2}^{g p}$ is injective.

Corollary 3.15. - Let $S$ be a fine log scheme and suppose given a chart $Q \rightarrow \mathcal{M}_{S}$. Let $U=\coprod_{(\theta, P)} \stackrel{\circ}{Q}_{Q}[P]$, where the disjoint union is taken over injective maps of fine monoids $\theta: Q \rightarrow P$. Then the natural map

$$
U \rightarrow \mathcal{L}_{S}
$$

is flat and surjective.

Proof. - The map (3.15.1) is surjective by (2.2) which shows that any 1-morphism $T \rightarrow \mathcal{L} \operatorname{og}_{S}$ factors fppf-locally on $T$ through one of the $\dot{S}_{Q}[P]$ appearing in the definition of $U$. This local factorization also implies that to verify the flatness of (3.15.1) it suffices to show that if $\theta: Q \rightarrow P$ and $\theta^{\prime}: Q \rightarrow P^{\prime}$ are two injective maps of fine monoids, then the fiber product

$$
\stackrel{\circ}{S}_{Q}[P] \times_{\mathcal{L o g}_{S}} \stackrel{\circ}{S}_{Q}\left[P^{\prime}\right] \simeq \stackrel{\circ}{S} \times_{\operatorname{Spec}(\mathbb{Z}[Q])}\left(\operatorname{Spec}(\mathbb{Z}[P]) \times \log _{\operatorname{Spec}(Q \rightarrow \mathbb{Z}[Q])} \operatorname{Spec}\left(\mathbb{Z}\left[P^{\prime}\right]\right)\right)
$$

is flat over both $\stackrel{\circ}{S}_{Q}[P]$ and $\stackrel{\circ}{S}_{Q}\left[P^{\prime}\right]$. This follows from (3.13).

\subsection{Completion of proof of (1.1)}

By (3.2) the diagonal

$$
\Delta: \mathcal{L}_{o_{S}} \rightarrow \mathcal{L}_{o}{ }_{S}{ }_{S} \mathcal{L} o_{S}
$$

is representable and of finite presentation. Thus by ([14], 10.1) it suffices to exhibit a flat cover, locally of finite presentation over $\stackrel{\circ}{S}$, of $\mathcal{L} o g_{S}$, since $\mathcal{L}_{o g_{S}}$ is a stack with respect to the fppf topology by (A.2). To find such a cover, we may replace $S$ by an étale cover and hence can assume that we have a chart $Q \rightarrow \mathcal{M}_{S}$. In this case, a cover of $\mathcal{L} o g_{S}$ with the desired properties is provided by (3.15).

Remark 3.17. - The stacks $\mathcal{L}_{\log }$ are not quasi-separated. Indeed this is equivalent to saying that the functors $I$ of (3.4(i)) are separated over $X$ which they are not (see (3.12)).

Example 3.18. - We continue with the example discussed in (3.11). Fix an integer $r \geqslant 1$, and let $\mathcal{S}$ be the fibered category over the category of schemes whose fiber over some scheme $T$ is the groupoid of fine $\log$ structure $\mathcal{M}$ on $T$ such that for every geometric point $\bar{t} \rightarrow T$ the stalk $\overline{\mathcal{M}}_{\bar{t}}$ is isomorphic to $\mathbb{N}^{r^{\prime}}$ for some $r^{\prime} \leqslant r$. If $\mathcal{M}$ is any fine $\log$ structure on $T$, then the set of points $t \in T$ for which $\overline{\mathcal{M}}_{\bar{t}}$ is isomorphic to $\mathbb{N}^{r^{\prime}}$ for some $r^{\prime} \leqslant r$ is an open set by (3.5(ii)) and (3.5(iii)). Hence $\mathcal{S}$ is an open substack of $\mathcal{L}_{\left(\operatorname{Spec}(\mathbb{Z}), \mathcal{O}_{\text {Spec }(\mathbb{Z})}^{*}\right)}$. However, we can see directly that $\mathcal{S}$ is an algebraic stack. 
If $\mathcal{M}$ is a fine $\log$ structure on a scheme $T$, then $\mathcal{M}$ defines an object of $\mathcal{S}(T)$ if and only if étale locally on $T$ there exists a chart $\mathbb{N}^{r} \rightarrow \mathcal{M}$. The "if" direction follows from (3.5(i)). As for the "only if" direction, note that if $\mathcal{M} \in \mathcal{S}(T)$, then we can by (2.1) étale locally find a chart $\beta: \mathbb{N}^{r^{\prime}} \rightarrow \mathcal{M}$ for some $r^{\prime} \leqslant r$. Defining $\beta_{2}: \mathbb{N}^{r} \simeq \mathbb{N}^{r^{\prime}} \oplus \mathbb{N}^{r-r^{\prime}} \rightarrow \mathcal{M}$ to be the map whose restriction to $\mathbb{N}^{r^{\prime}}$ is $\beta$ and whose restriction to $\mathbb{N}^{r-r^{\prime}}$ sends all elements to $\lambda(1)$, we obtain a chart as desired for $\mathcal{M}$.

Let $X=\operatorname{Spec}\left(\mathbb{Z}\left[X_{1}, \ldots, X_{r}\right]\right)$, and let $\mathcal{M}_{X}$ be the log structure on $X$ defined in (3.11.1). By the preceding paragraph, any 1-morphism $t: T \rightarrow \mathcal{S}$ factors étale locally on $T$ through $X$. Therefore, given two 1-morphisms $t: T \rightarrow \mathcal{S}$ and $t^{\prime}: T^{\prime} \rightarrow \mathcal{S}$ the fiber product $T \times{ }_{\mathcal{S}} T^{\prime}$ is representable by an algebraic space. Indeed this can be verified étale locally on $T$ and $T^{\prime}$, and so we may assume that $t$ and $t^{\prime}$ factor through maps $\tilde{t}: T \rightarrow X$ and $\tilde{t}^{\prime}: T^{\prime} \rightarrow X$. In this case,

$$
T \times_{X}\left(X \times_{\mathcal{S}} X\right)_{X} T^{\prime}
$$

is representable since $X \times_{\mathcal{S}} X$ is representable by (3.11).

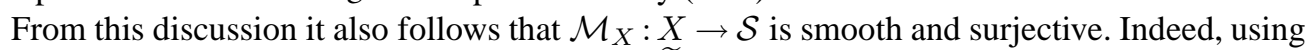
the notation of (3.11), the two projections from $\widetilde{I}$ to $X$ are smooth and surjective by (3.11.2), and since $\widetilde{I} \rightarrow I$ is étale, the two projections from $I$ to $X$ are also smooth and surjective. Now if $t: T \rightarrow \mathcal{S}$ is any 1-morphism, to verify that $T \times_{\mathcal{S}} X \rightarrow T$ is smooth and surjective, we may replace $T$ by an étale cover and hence can assume that $t$ factors through a map $\tilde{t}: T \rightarrow X$. In this case, $T \times_{\mathcal{S}} X$ is isomorphic to $T \times_{X} I$ and the result follows. This proves that $\mathcal{S}$ is an algebraic stack.

We conclude this section by noting two basic properties of the stacks $\log _{S}$.

PROPOSITION 3.19. - Let $s: \stackrel{\circ}{S} \rightarrow \mathcal{L}_{\text {og }}$ be the map induced by the morphism of log schemes id $: S \rightarrow S$.

(i) For any morphism of log schemes $f: T \rightarrow S$, the fiber product $\stackrel{\circ}{T} \times \mathcal{L}_{\text {og }} S \stackrel{\circ}{S}$ represents the functor on $\stackrel{\circ}{T}$-schemes which to any $g: Z \rightarrow \stackrel{\circ}{T}$ associates the unital set if

$$
g^{*}\left(f^{b}\right): g^{*} f^{*} \mathcal{M}_{S} \rightarrow g^{*} \mathcal{M}_{T}
$$

is an isomorphism and the empty set otherwise.

(ii) The map $s$ is an open immersion.

Proof. - Statement (i) follows from the definition of $\stackrel{\circ}{T} \times \log _{S} \stackrel{\circ}{S}$.

To see (ii), note that a morphism of fine $\log$ structures $\mathcal{M}_{1} \rightarrow \mathcal{M}_{2}$ on a scheme $X$ is an isomorphism if and only if the induced map $\overline{\mathcal{M}}_{1} \rightarrow \overline{\mathcal{M}}_{2}$ is an isomorphism ([8], 3.2), so we can interpret the functor $\stackrel{\circ}{T} \times{ }_{\mathcal{L} o g_{S}} \stackrel{\circ}{S}$ as the functor on $\stackrel{\circ}{T}$-schemes which to any $g: Z \rightarrow \stackrel{\circ}{T}$ associates the unital set if the map $g^{-1} f^{-1} \overline{\mathcal{M}}_{S} \rightarrow g^{-1} \overline{\mathcal{M}}_{T}$ is an isomorphism and the empty set otherwise. Now the set of points $t \in \stackrel{\circ}{\mathrm{o}}$ for which the map $\left(f^{-1} \overline{\mathcal{M}}_{S}\right)_{\bar{t}} \rightarrow \overline{\mathcal{M}}_{T, \bar{t}}$ is an isomorphism is a constructible set by (3.5(ii)) and is stable under generization by (3.5(iii)); hence is an open set. From this (ii) follows.

Proposition 3.20. - Suppose

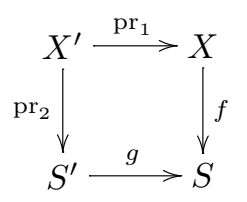


is a cartesian diagram in the category of fine log schemes ([9], 2.6). Then the induced diagram of algebraic stacks

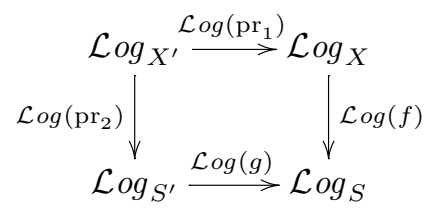

is also cartesian ([14], 2.2.2).

Proof. - By the definition of the fiber product of stacks ([14], 2.2.2), there is a natural functor

$$
\mathcal{L} \operatorname{og}_{X^{\prime}} \rightarrow \mathcal{L} \operatorname{og}_{S^{\prime}} \times \log _{S} \mathcal{L} \operatorname{og}_{X}
$$

and it follows from the definitions that this functor is fully faithful. To see that it is essentially surjective, suppose $T$ is a scheme and that we are given an object $O \in\left(\mathcal{L}_{\operatorname{og}_{S^{\prime}}} \times_{\mathcal{L}_{o g}} \mathcal{L}_{\log _{X}}\right)(T)$. By definition, $O$ is a collection of data consisting of morphisms $g_{1}: T \rightarrow \stackrel{S}{S}^{\prime}$ and $g_{2}: T \rightarrow X$ such that $\stackrel{\circ}{g} \circ g_{1}=\stackrel{\circ}{f} \circ g_{2}$ (call this morphism $h$ ), together with fine log structures $\mathcal{M}_{1}$ and $\mathcal{M}_{2}$ on $T$ and a commutative diagram

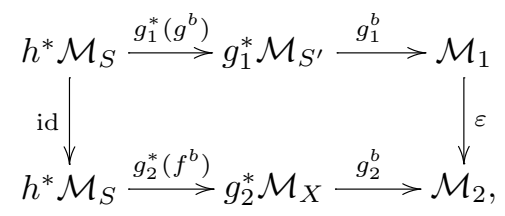

where $\varepsilon$ is an isomorphism. Let $O^{\prime} \in \mathcal{L} o g_{X^{\prime}}$ be the morphism $\left(T, \mathcal{M}_{1}\right) \rightarrow X^{\prime}$ obtained from the morphisms

$$
\left(g_{2}, \varepsilon^{-1} \circ g_{2}^{b}\right):\left(T, \mathcal{M}_{1}\right) \rightarrow X, \quad\left(g_{1}, g_{1}^{b}\right):\left(T, \mathcal{M}_{1}\right) \rightarrow S^{\prime} .
$$

Then the image of $O^{\prime}$ under (3.20.2) is isomorphic to $O$ and so (3.20.2) is essentially surjective.

\section{Properties of morphisms of fine log schemes}

Let $f: X \rightarrow S$ be a morphism of fine $\log$ schemes. If $g: Z \rightarrow X$ is an object of $\log _{X}$, then $f \circ g: Z \rightarrow S$ is an object of $\mathcal{L}_{o g}$, and hence there is a natural functor

$$
\mathcal{L} o g(f): \mathcal{L}_{\operatorname{og}} \rightarrow \mathcal{L} \operatorname{og}_{S}
$$

This functor is faithful, and hence by ([14], 8.1.2) the morphism of stacks $\mathcal{L} o g(f)$ is a representable.

DEFINITION 4.1. - Let $\mathcal{P}$ be a property of representable morphisms of algebraic stacks. We say that $f: X \rightarrow S$ has property $\mathcal{L} o g(\mathcal{P})$ if the morphism of stacks $\mathcal{L} o g(f)$ has property $\mathcal{P}$. We say that $f$ has property weak $\log (\mathcal{P})$ if the map $X \rightarrow \mathcal{L}^{\circ} g_{S}$ has property $\mathcal{P}$.

For example, the property $\mathcal{P}$ could be the property of being étale, smooth, flat, or locally of finite presentation ([14], 3.10). We will often refer to $f$ as being (weakly) $\mathcal{L}$ og étale, (weakly) $\mathcal{L}$ og smooth, (weakly) $\mathcal{L}$ og flat, or (weakly) $\mathcal{L}$ og locally of finite presentation. In this section we 
study the relationship between these notions and K. Kato's notions of log étale, log smooth, and $\log$ flat morphisms of log schemes (([9], 3.3) and ([10], 1.10)).

Remark 4.2. - If the property $\mathcal{P}$ is preserved under restriction to open substacks, then (3.19(ii)) implies that $f$ has property weak $\mathcal{L} \operatorname{og}(\mathcal{P})$ if $f$ has property $\log (\mathcal{P})$. As the following example shows, however, there exist properties $\mathcal{P}$ for which the condition weak $\log (\mathcal{P})$ does not imply $\operatorname{L} o g(\mathcal{P})$.

Example 4.3. - Let $R$ be a ring and let $(\mathcal{M}, \alpha)$ (or just $\mathcal{M}$ ) be the log structure associated to the pre-log structure $\mathbb{N} \rightarrow R, 1 \mapsto 0$. Then the morphism

$$
\mathcal{M}: \operatorname{Spec}(R) \rightarrow \mathcal{L}_{o g}\left(\operatorname{Spec}(R), \mathcal{O}_{R}^{*}\right)
$$

induced by $\mathcal{M}$ has geometrically connected fibers. Indeed if $T=\operatorname{Spec}(k)$ is the spectrum of a separably closed field over $R$ and $\mathcal{M}_{k}$ a fine log structure on $T$ defining a map

$$
T \rightarrow \mathcal{L}_{o g}\left(\operatorname{Spec}(R), \mathcal{O}_{R}^{*}\right),
$$

then the product $\operatorname{Spec}(R) \times \log _{\left(\operatorname{Spec}(R), \mathcal{O}_{R}^{*}\right)} T$ is the empty scheme unless $\overline{\mathcal{M}}_{k}$ is isomorphic to $\mathbb{N}$ in which case $\mathcal{M}_{k}$ and $\left.\mathcal{M}\right|_{\text {Spec }(k)}$ are isomorphic ([9], 2.5.2). If $\mathcal{M}_{k}$ and $\left.\mathcal{M}\right|_{\operatorname{Spec}(k)}$ are isomorphic, then (3.10) shows that the set of isomorphism between them is a (trivial) $\mathbb{G}_{m}$-torsor (and in particular is connected).

On the other hand, the morphism

$$
\mathcal{L}_{o g_{(\operatorname{Spec}(R), \mathcal{M})}} \rightarrow \mathcal{L}_{\left.o g_{(\operatorname{Spec}}(R), \mathcal{O}_{R}^{*}\right)}
$$

does not have geometrically connected fibers. For example, if $f: T \rightarrow \operatorname{Spec}(R)$ is an $R$-scheme, then lifting $f$ to a $T$-valued point of the product

$$
\operatorname{Spec}(R) \times_{\mathcal{M}, \mathcal{L}_{\left(\operatorname{Spec}(R), \mathcal{O}_{R}^{*}\right)}} \mathcal{L}_{\operatorname{og}_{(\operatorname{Spec}(R), \mathcal{M})}}
$$

is equivalent to extending $f$ to a morphism of log schemes

$$
\left(f, f^{b}\right):\left(T, f^{*} \mathcal{M}\right) \rightarrow(\operatorname{Spec}(R), \mathcal{M}) .
$$

Now giving the map $f^{b}$ is equivalent to giving a map $\mathbb{N} \rightarrow \alpha^{-1}(0) \subset f^{*} \mathcal{M}$ and hence (4.3.1) is isomorphic to $\mathbb{G}_{m} \times\{\mathbb{N}-\{0\}\}$. In particular (4.3.1) is not geometrically connected over $R$.

Recall the following definitions, due to K. Kato, of log smooth and log étale morphisms ([9], 3.3):

Definition 4.4. - A morphism of fine log schemes $f: X \rightarrow S$ is formally log smooth (resp. formally log étale) if for every commutative diagram of solid arrows in the category of fine log schemes

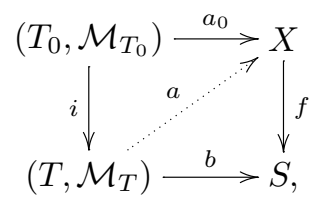


where $i$ is a strict closed immersion defined by a square zero ideal $I$, there exists étale locally on $T_{0}$ a dotted arrow $a$ (resp. a unique dotted arrow $a$ ) filling in the diagram. The morphism $f$ is $\log$ smooth (resp. log étale) if it is formally log smooth (resp. formally log étale) and $f$ is locally of finite presentation.

In order to relate the notion of a log smooth (resp. log étale) morphism to the notion of a $\mathcal{L} o g$ smooth (resp. $\mathcal{L}$ og étale) morphism we need the notion of a formally smooth (resp. formally étale) morphism of algebraic stacks:

DEFINITION 4.5. - Let $F: \mathfrak{X} \rightarrow \mathfrak{Y}$ be a representable morphism of algebraic stacks. Then $F$ is formally smooth (resp. formally étale) if for every 2-commutative diagram of solid arrows

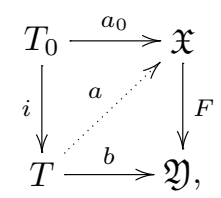

where $i$ is a closed immersion defined by a square zero ideal $I$, there exists étale locally on $T_{0}$ a dotted arrow $a$ (resp. a unique dotted arrow $a$ ) filling in the diagram.

The following is our main result about (weakly) $\mathcal{L} o g$ étale, (weakly) $\mathcal{L} o g$ smooth, and (weakly) $\mathcal{L}$ og flat morphisms:

THEOREM 4.6. - Let $f: X \rightarrow S$ be a morphism of fine log schemes.

(i) $f$ is formally log smooth (resp. formally log étale) if and only if for every commutative diagram as in (4.4.1) with $a_{0}$ strict there exists étale locally on $T_{0}$ a map a (resp. a unique map a) filling in the diagram.

(ii) $f$ is $\mathcal{L}$ og smooth (resp. $\mathcal{L}$ og étale) if and only if $f$ is locally of finite presentation and $\mathcal{L} o g(f)$ is formally smooth (resp. formally étale), and this is also equivalent to $f$ being log smooth (resp. log étale).

(iii) $f$ is $\mathcal{L}$ og smooth (resp. $\mathcal{L}$ og étale) if and only if $f$ is weakly $\mathcal{L}$ og smooth (resp. weakly $\mathcal{L}$ og étale).

(iv) $f$ is $\mathcal{L}$ og flat if and only if fppf locally there exists a chart $\left(Q, P, \beta_{Q}, \beta_{P}, \theta\right)$ for $f$ such that $\theta^{g p}: Q^{g p} \rightarrow P^{g p}$ is injective, and the map

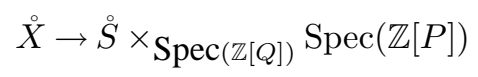

is flat in the usual sense.

(v) $f$ is $\mathcal{L}$ og flat if and only if $f$ is weakly $\mathcal{L}$ og flat.

Remark 4.7. - Statements (4.6(iv)) and (4.6(v)) imply that the notion of (weakly) $\mathcal{L}$ og flat morphism is equivalent to the notion of $\log$ flat morphism in the sense of K. Kato ([10], 1.10).

Proof of (4.6). - The "only if" direction of (i) is clear. To prove the "if" direction, suppose that for every diagram as in (4.4.1) with $a_{0}$ strict, there exists étale locally on $T_{0}$ a morphism $a$ (resp. a unique morphism $a$ ) filling in the diagram. We have to show that given any diagram as in (4.4.1) there exists étale locally on $T_{0}$ a morphism (resp. a unique morphism) $a$ filling in the diagram. To see this, let

$$
\mathcal{N}:=\mathcal{M}_{T} \times \mathcal{M}_{T_{0}} a_{0}^{*} \mathcal{M}_{X}
$$

Then $\mathcal{N}$ with its natural map to $\mathcal{O}_{T}$ is a log structure on $T$ lifting $a_{0}^{*} \mathcal{M}_{X}$ to $T$, and $\mathcal{N}$ is easily seen to be fine. In fact the fine log scheme $(T, \mathcal{N})$ is the co-product in the category of log schemes 
of the diagram

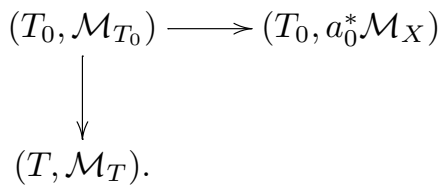

From this it follows that the existence (resp. existence and uniqueness) of the arrow $a$ is equivalent to the existence of an arrow $a^{\prime}$ (resp. existence of a unique arrow $a^{\prime}$ ) filling in the diagram

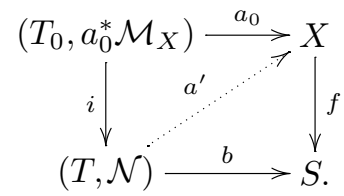

By assumption there exists étale locally on $T_{0}$ such an arrow (resp. a unique such arrow), and hence (i) follows.

To see (ii) and (iii), note that by the definition of $\mathcal{L}_{\operatorname{og}}$ and $\mathcal{L}_{\log }$, to give a diagram as in (4.4.1) is equivalent to giving a diagram

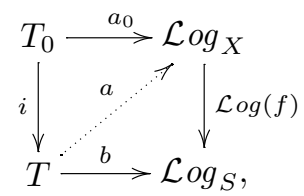

and finding a morphism $a$ filling in diagram (4.4.1) is equivalent to finding a morphism $a$ filling in (4.7.1). Moreover, to give a diagram as in (4.4.1) with $a_{0}$ strict is equivalent, by (3.19(i)), to giving a diagram as in (4.7.1) for which the morphism $a_{0}$ factors through the canonical open immersion $X \hookrightarrow \mathcal{L} g_{X}$ discussed in (3.19). Thus $f$ is formally log smooth (resp. formally log étale) if and only if $\mathcal{L} o g(f)$ is formally smooth (resp. formally étale), and this in turn is by (4.6(i)) equivalent to the morphism $X \rightarrow \mathcal{L}_{o g}$ being formally smooth (resp. formally étale). Statements (4.6(ii)) and (4.6(iii)) therefore follow from the following two lemmas.

LEMMA 4.8. - The following are equivalent.

(i) $f$ is $\mathcal{L}$ og locally of finite presentation.

(ii) $f$ is weakly $\mathcal{L}$ og locally of finite presentation.

(iii) $\stackrel{\circ}{f}: \stackrel{\circ}{X} \rightarrow \stackrel{\circ}{S}$ is locally of finite presentation.

Proof. - Let $T \rightarrow \mathcal{L}_{o g}$ (resp. $\widetilde{S} \rightarrow \mathcal{L} o g_{S}$ ) be a smooth cover locally of finite presentation

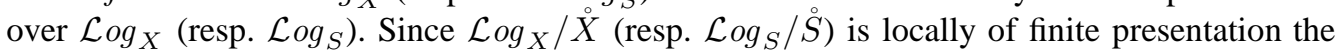
map $T \rightarrow X$ (resp. $\widetilde{S} \rightarrow \stackrel{\circ}{S}$ ) is also locally of finite presentation.

(i) implies (ii). If $\mathcal{L} \operatorname{og}(f): \mathcal{L}_{o g} \rightarrow \mathcal{L} \operatorname{og}_{S}$ is locally of finite presentation, then since the canonical section $s: X \rightarrow \mathcal{L}^{\circ} g_{X}$ is an open immersion by (3.19(ii)), the map $X \rightarrow \mathcal{L}^{\circ} g_{S}$ is also locally of finite presentation.

(ii) implies (iii). If $\stackrel{\circ}{X} \rightarrow \mathcal{L}_{o} g_{S}$ is locally of finite presentation, then the composite

$$
\stackrel{\circ}{X} \rightarrow \mathcal{L}^{\circ} g_{S} \rightarrow \stackrel{\circ}{S}
$$

is also locally of finite presentation, since $\mathcal{L} \operatorname{og}_{S} \rightarrow \stackrel{\circ}{S}$ is locally of finite presentation by (1.1). 


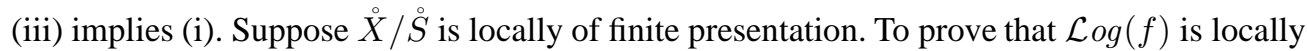
of finite presentation it suffices by ([14], 4.14) to show that the map $T \times \log _{S} \widetilde{S} \rightarrow \widetilde{S}$ is locally of finite presentation. The product $T \times \log _{S} \widetilde{S}$ is isomorphic to the algebraic space $I$ over $T \times{ }_{S} \widetilde{S}$ obtained from (3.4(i)) applied to the diagram

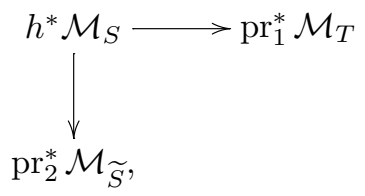

where $h: T \times{ }_{S} \widetilde{S} \rightarrow \stackrel{S}{ }$ denotes the structure morphism. By (3.4(i)), $I$ is of finite presentation

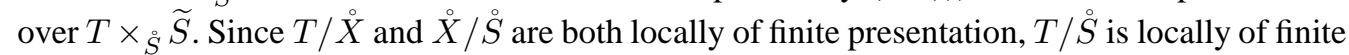
presentation and so the composite

$$
I \rightarrow T \times_{S} \widetilde{S} \rightarrow \widetilde{S}
$$

is also locally of finite presentation.

LEMMA 4.9. - Let $\mathfrak{X}$ and $\mathfrak{Y}$ be algebraic stacks over $\stackrel{\circ}{S}$, and let $F: \mathfrak{X} \rightarrow \mathfrak{Y}$ be a representable morphism locally of finite presentation.

(i) If $Y \rightarrow \mathfrak{Y}$ is a smooth cover and $F_{Y}: Y_{\mathfrak{X}} \rightarrow Y$ the base change of $F$, then $F$ is formally étale if and only if $F_{Y}$ is formally étale.

(ii) $F$ is smooth (resp. étale) if and only if $F$ is formally smooth (resp. formally étale).

Proof. - For (i), the "only if" direction is immediate so let us verify the "if" direction. Suppose given a commutative diagram as in (4.5.1). Then to show that there exists a unique arrow $a$ filling in the diagram, we may replace $T_{0}$ by an étale cover since $\mathfrak{X}$ and $\mathfrak{Y}$ are stacks with respect to the étale topology. Now since $Y / \mathfrak{Y}$ is smooth, there exists étale locally on $T_{0}$ a factorization of $b$ through $Y$ ([4], IV.17.16.3), and hence it suffices to verify the infinitesimal lifting property for diagrams (4.5.1) where $b$ factors through a map $\tilde{b}: T \rightarrow Y$. But in this case finding a dotted arrow $a$ filling in diagram (4.5.1) is equivalent to finding a dotted arrow filling in the following diagram:

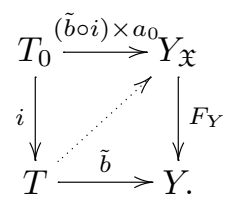

Thus if $F_{Y}$ is formally étale there exists étale locally on $T_{0}$ a unique arrow as desired.

As for (ii), the statement that $F$ is smooth if and only if $F$ is formally smooth follows from ([14], 4.15).

Now it follows from ([4], IV.17.3.1) and the assumption that $F$ is locally of finite presentation, that for any smooth cover $Y / \mathfrak{Y}$, the morphism $F_{Y}: Y_{\mathfrak{X}} \rightarrow Y$ is étale if and only if $F_{Y}$ is formally étale. Therefore, by definition of an étale morphism of stacks ([14], 3.10.1), $F$ is étale if and only if $F_{Y}$ is formally étale, and hence (i) implies that $F$ is étale if and only if $F$ is formally étale.

Since $\mathcal{L}$ og flat implies weakly $\mathcal{L} o g$ flat by (4.2), statements (4.6(iv)) and (4.6(v)) follow from the following two lemmas.

LEMma 4.10. - If $f$ is weakly $\mathcal{L}$ og flat, then locally in the fppf topology there exists a chart as in (4.6(iv)). 
Proof. - We can replace $\stackrel{\circ}{S}$ by an étale cover, and so may assume that we have a chart $Q \rightarrow \mathcal{M}_{S}$. Let

$$
U=\coprod_{(P, \theta)} \stackrel{\circ}{S}_{Q}[P] \rightarrow \mathcal{L} g_{S}
$$

be the flat cover constructed in (3.15). We then obtain a diagram

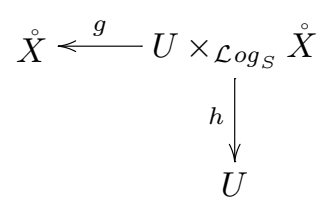

where $g$ is an fppf cover and $h$ is flat. By definition of $U$ this means that locally in the fppftopology on $X_{X}$ we have a chart as in (4.6(iv)).

LEMMA 4.11. - If fppf locally there exists a chart as in (4.6(iv)), then $f$ is $\mathcal{L}$ og flat.

Proof. - To verify that $\mathcal{L}_{o g} \rightarrow \mathcal{L} \log _{S}$ is flat we may replace $\stackrel{\circ}{S}$ and $\stackrel{\circ}{X}$ by fppf covers, and hence we can assume that we have a global chart as in (4.6(iv)). Let

$$
U_{X}=\coprod_{\left(P^{\prime}, \theta\right)} \stackrel{\circ}{X}_{P}\left[P^{\prime}\right] \rightarrow \mathcal{L} \log _{X}
$$

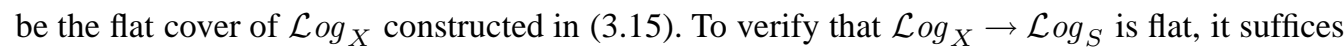
to show that an fppf cover of $\mathcal{L}_{o g}$ is flat over $\mathcal{L} o g_{S}$, and hence it is enough to show that each of the $\dot{X}_{P}\left[P^{\prime}\right]$ are flat over $\mathcal{L}_{\log }$. To see this, consider the commutative diagram

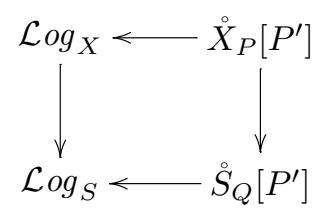

where $\stackrel{\circ}{S}_{Q}\left[P^{\prime}\right]$ is the scheme obtained from the composite $Q \rightarrow P \rightarrow P^{\prime}$. The map

$$
\stackrel{\circ}{S}_{Q}\left[P^{\prime}\right] \rightarrow \mathcal{L}^{\circ} g_{S}
$$

is flat by Theorem (3.13), and so is the map

$$
\stackrel{\circ}{X}_{P}\left[P^{\prime}\right] \rightarrow \stackrel{\circ}{S}_{Q}\left[P^{\prime}\right]
$$

since there is a natural cartesian diagram

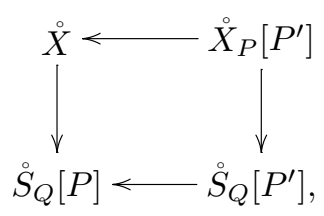


and the map $\stackrel{\circ}{X} \rightarrow \stackrel{\circ}{S}_{Q}[P]$ is flat by assumption. Consequently the composite $X_{P}\left[P^{\prime}\right] \rightarrow \mathcal{L}_{o g}$ is also flat, and hence $\mathcal{L} \operatorname{og}_{X} \rightarrow \mathcal{L}_{o g}$ is flat.

This concludes the proof of Theorem (4.6).

From (4.6) we obtain some basic facts about log flat morphisms in the sense of K. Kato ([10], 1.10 ). Note that by (4.7) a morphism of fine $\log$ schemes $f: X \rightarrow S$ is $\log$ flat if and only if it is $\mathcal{L}$ og flat.

COROLlaRY 4.12.- (i) If

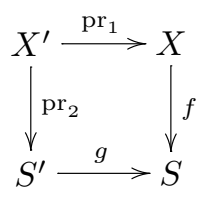

is a cartesian diagram in the category of fine log schemes with $f$ log flat, then $\mathrm{pr}_{2}$ is log flat.

(ii) Suppose given morphisms of fine log schemes

$$
X \stackrel{f}{\rightarrow} Y \stackrel{g}{\rightarrow} Z
$$

and suppose that $f$ and $g$ are both log flat. Then $g \circ f$ is also log flat.

Proof. - To say that the map $\mathrm{pr}_{2}$ in (i) is $\log$ flat is equivalent to saying that

$$
\mathcal{L} o g\left(\mathrm{pr}_{2}\right): \mathcal{L} \operatorname{og}_{X^{\prime}} \rightarrow \mathcal{L}_{\log _{S^{\prime}}}
$$

is flat which follows from (3.20).

Since $g \circ f$ is $\log$ flat if and only if the composite

$$
\mathcal{L} \circ g(g \circ f)=\mathcal{L} \circ g(g) \circ \mathcal{L} \circ g(f): \mathcal{L}_{o g} \rightarrow \mathcal{L}^{\circ} g_{Z}
$$

is flat, statement (ii) holds since $\mathcal{L} o g(f)$ and $\mathcal{L} o g(g)$ are flat.

Finally we mention a definition and two theorems of K. Kato which give a stronger result than (4.6(iv)) about the relationship between log flatness and charts. The proofs of these results are outside the scope of this paper.

Definition 4.13 ([11], Definition 1). - Let $f: X \rightarrow S$ be a morphism of fine log schemes, and suppose given a chart $\left(Q, P, \beta_{Q}, \beta_{P}, \theta\right)$ for $f$. Then this chart is called neat at a geometric point $\bar{x} \rightarrow X$ if the following two conditions hold:

(i) The map $\theta^{g p}: Q^{g p} \rightarrow P^{g p}$ is injective.

(ii) The natural map

$$
\operatorname{Coker}\left(Q^{g p} \rightarrow P^{g p}\right) \rightarrow \operatorname{Coker}\left(\overline{\mathcal{M}}_{S, f(\bar{x})}^{g p} \rightarrow \overline{\mathcal{M}}_{X, \bar{x}}^{g p}\right)
$$

is an isomorphism.

The basic existence result about neat charts is the following theorem (4.14), and the relationship between neat charts and log flatness is given in (4.15).

THEOREM 4.14 ([11], Proposition 1). - Let $f: X \rightarrow$ S be a morphism of fine log schemes, and suppose $\beta_{Q}: Q \rightarrow \mathcal{M}_{S}$ is a chart. Then in a fppf neighborhood of any point $x \in X$, there exists a chart $\left(Q, P, \beta_{Q}, \beta_{P}, \theta\right)$ (with the same $\left(Q, \beta_{Q}\right)$ ) which is neat at a geometric point over $x$. 
THEOREM 4.15 ([11], Proposition 2). - Let $f: X \rightarrow S$ be a morphism of fine log schemes with $f$ locally of finite presentation and let $x \in X^{\circ}$ be a point. Suppose given a chart $\left(Q, P, \beta_{Q}, \beta_{P}, \theta\right)$ for $f$ which is neat at a geometric point over $x$. Then $f$ is log flat at $x$ if and only if the map of schemes

$$
\stackrel{\circ}{X} \rightarrow \stackrel{\circ}{S}_{Q}[P]
$$

induced by the chart is flat at $x$.

\section{The local structure of $\log _{S}$}

In this section we explain how work of L. Illusie on "toric stacks" can be used to construct an étale cover of the stack $\log _{S}$ (where $S$ is a fine log scheme). The main results of this Section were discovered by L. Illusie in somewhat different language, and we are grateful to him for allowing us to include them here.

Before discussing toric stacks, however, we need some foundational definitions and results about log structures on algebraic stacks.

If $\mathcal{S}$ is an algebraic stack, we denote by $\operatorname{Lis}-\operatorname{Et}(\mathcal{S})$ the lisse-étale site of $\mathcal{S}$ ([14], 12.1(i)). Recall that the objects of $\operatorname{Lis}-\operatorname{Et}(\mathcal{S})$ are pairs $(U, u)$, where $U$ is an algebraic space and $u: U \rightarrow \mathcal{S}$

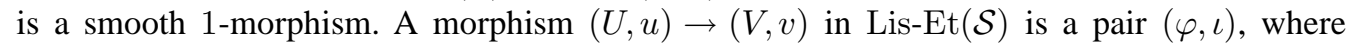
$\varphi: U \rightarrow V$ is a morphism of algebraic spaces and $\iota: u \rightarrow v \circ \varphi$ is a 2 -isomorphism. A collection of maps $\left\{\left(\varphi_{i}, \iota_{i}\right):\left(U_{i}, u_{i}\right) \rightarrow(U, u)\right\}$ is a covering if the map

$$
\coprod_{i} \varphi_{i}: \coprod_{i} U_{i} \rightarrow U
$$

is étale and surjective. There is a natural sheaf of $\operatorname{rings} \mathcal{O}_{\mathcal{S}_{\text {lis-et }}}$ on $\operatorname{Lis-\operatorname {Et}}(\mathcal{S})$ defined by

$$
\mathcal{O}_{\mathcal{S}_{\text {lis-et }}}((U, u))=\Gamma\left(U, \mathcal{O}_{U}\right)
$$

We denote by $\left(\mathcal{S}_{\text {lis-et }}, \mathcal{O}_{\mathcal{S}_{\text {lis-et }}}\right)$ the associated ringed topos.

Definition 5.1. - A pre-log structure on an algebraic stack $\mathcal{S}$ is a pair $(\mathcal{M}, \alpha)$ (often denoted just $\mathcal{M}$ ), where $\mathcal{M}$ is a sheaf of monoids on $\operatorname{Lis-\operatorname {Et}}(\mathcal{S})$ and $\alpha: \mathcal{M} \rightarrow \mathcal{O}_{\mathcal{S}_{\text {lis-et }}}$ is a morphism of sheaves of monoids. A pre-log structure $(\mathcal{M}, \alpha)$ is a log structure if the map $\alpha^{-1}\left(\mathcal{O}_{\mathcal{S}_{\text {lis-et }}^{*}}\right) \rightarrow \mathcal{O}_{\mathcal{S}_{\text {lis-et }}}^{*}$ is bijective. A $\log$ structure $\mathcal{M}$ on $\mathcal{S}$ is fine if for every object $(U, u) \in \operatorname{Lis}-e t(\mathcal{S})$, the restriction $\left.\mathcal{M}\right|_{U_{\text {et }}}$ is a fine log structure on $U$ and for every morphism $f:(U, u) \rightarrow\left(U^{\prime}, u^{\prime}\right)$ in Lis-Et $(\mathcal{S})$ the natural map $\left.f^{*}\left(\left.\mathcal{M}\right|_{U_{\text {et }}^{\prime}}\right) \rightarrow \mathcal{M}\right|_{U_{\text {et }}}$ is an isomorphism. A log algebraic stack is a pair $\left(\mathcal{S}, \mathcal{M}_{\mathcal{S}}\right)$, where $\mathcal{S}$ is an algebraic stack and $\mathcal{M}_{\mathcal{S}}$ is a log structure on $\mathcal{S}$.

Remark 5.2. - Just as in ([9], 1.3), if $\mathcal{M}$ is a pre-log structure on an algebraic stack $\mathcal{S}$, then there is a universal map $\mathcal{M} \rightarrow \mathcal{M}^{a}$ from $\mathcal{M}$ to a log structure.

By the same argument as in ([14], 12.2.1), the category of fine log structures on an algebraic stack $\mathcal{S}$ is equivalent to the category of systems $\left(\mathcal{M}_{(U, u)}, \psi_{(\varphi, \iota)}\right)$ consisting of a fine log structure $\mathcal{M}_{(U, u)}$ on $U$ for each $(U, u) \in \operatorname{Lis-\operatorname {Et}}(\mathcal{S})$ and an isomorphism $\psi_{(\varphi, \iota)}: \varphi^{*} \mathcal{M}_{(V, v)} \rightarrow \mathcal{M}_{(U, u)}$ for each morphism $(\varphi, \iota):(U, u) \rightarrow(V, v)$ in $\operatorname{Lis-\operatorname {Et}}(\mathcal{S})$ such that for a composite

$$
(U, u) \stackrel{(\varphi, \iota)}{\longrightarrow}(V, v) \stackrel{\left(\varphi^{\prime}, \iota^{\prime}\right)}{\longrightarrow}(W, w)
$$


the two isomorphisms

$$
\psi_{\left(\varphi^{\prime} \circ \varphi, \varphi^{*}\left(\iota^{\prime}\right) \circ \iota\right)}, \psi_{(\varphi, \iota)} \circ \varphi^{*} \psi_{\left(\varphi^{\prime}, \iota^{\prime}\right)}: \varphi^{*} \varphi^{\prime *} \mathcal{M}_{(W, w)} \rightarrow \mathcal{M}_{(U, u)}
$$

are equal.

In particular, if $\mathcal{S}$ is an algebraic space and $\mathcal{M}$ is a fine log structure on $\mathcal{S}_{\text {et }}$, then we obtain a fine $\log$ structure $\mathcal{M}^{\text {lis-et }}$ on $\operatorname{Lis-\operatorname {Et}}(\mathcal{S})$, by defining $\mathcal{M}_{(U, u)}:=u^{*} \mathcal{M}$ for each $(U, u) \in$ Lis-Et $(\mathcal{S})$ (together with the natural transition maps $\psi_{(\varphi, \iota)}$ ). The following proposition follows from the same reasoning used in the proof of ([14], 12.3.3):

Proposition 5.3. - If $\mathcal{S}$ is an algebraic space, the functor $\mathcal{M} \mapsto \mathcal{M}^{\text {lis-et }}$ induces an equivalence of categories between the category of fine log structures on $\mathcal{S}_{\text {et }}$ and the category of fine log structures on $\mathcal{S}_{\text {lis-et. }}$ A quasi-inverse is given by the functor which restricts a fine log structure on $\mathcal{S}_{\text {lis-et }}$ to $\mathcal{S}_{\text {et }}$.

If $f: \mathcal{S}_{1} \rightarrow \mathcal{S}_{2}$ is a 1-morphism between two algebraic stacks, then $f$ extends naturally to a morphism of ringed topoi $\left(f, \theta_{f}\right):\left(\mathcal{S}_{1, \text { lis-et }}, \mathcal{O}_{\mathcal{S}_{1, \text { lis-et }}}\right) \rightarrow\left(\mathcal{S}_{2, \text { lis-et }}, \mathcal{O}_{\mathcal{S}_{2, \text { lis-et }}}\right)$ ([14], 12.9.3), where $\theta_{f}: f^{-1} \mathcal{O}_{\mathcal{S}_{2, \text { lis-et }}} \rightarrow \mathcal{O}_{\mathcal{S}_{1, \text { lis}}-\mathrm{et}}$ is a morphism of sheaves of rings. Hence if $\mathcal{M}$ is a pre-log structure on $\mathcal{S}_{2}$, we can define the pullback pre-log structure $f^{-1} \mathcal{M}$ to be

$$
f^{-1} \mathcal{M} \stackrel{f^{-1}(\alpha)}{\longrightarrow} f^{-1} \mathcal{O}_{\mathcal{S}_{2, \text { lis-et }}} \stackrel{\theta_{f}}{\longrightarrow} \mathcal{O}_{\mathcal{S}_{1, \text { lis-et }}} .
$$

If $\mathcal{M}$ is a $\log$ structure on $\mathcal{S}_{2}$, then we define its pullback $f^{*} \mathcal{M}$ to be the log structure associated to the pre-log structure $f^{-1}(\mathcal{M})$.

If $\mathcal{M}$ is a fine $\log$ structure on $\mathcal{S}_{2}$, then the pullback $f^{*} \mathcal{M}$ has a more concrete description. If $\left(U_{1}, u_{1}\right) \in \operatorname{Lis}-\operatorname{Et}\left(\mathcal{S}_{1}\right)$, then we can, after replacing $U_{1}$ by an étale cover find a 2 -commutative diagram

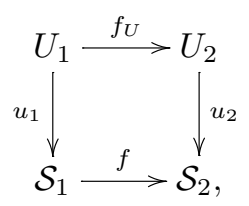

where $\left(U_{2}, u_{2}\right)$ is an object of $\operatorname{Lis}-\operatorname{Et}\left(\mathcal{S}_{2}\right)$. In this case, it follows from the construction of the functor $f^{-1}([14], 12.5)$ that the restriction $\left(f^{*} \mathcal{M}\right)_{\left(U_{1}, u_{1}\right)}$ of $f^{*} \mathcal{M}$ to $U_{1}$ is simply $f_{U}^{*}\left(\mathcal{M}_{\left(U_{2}, u_{2}\right)}\right)$. In particular, $f^{*} \mathcal{M}$ is again fine.

Remark 5.4. - If $f: T \rightarrow \mathcal{S}$ is a 1-morphism from a scheme $T$ to an algebraic stack $\mathcal{S}$, then if $\mathcal{M}$ is a fine $\log$ structure on $\mathcal{S}$ the pullback $f^{*} \mathcal{M}$ is a fine $\log$ structure on $T_{\text {lis-et. }}$. By (5.3), $f^{*} \mathcal{M}$ is isomorphic to $\mathcal{N}^{\text {lis-et }}$ for a unique fine $\log$ structure $\mathcal{N}$ on $T_{\text {et }}$. We often abuse notation and write $f^{*} \mathcal{M}$ for the fine $\log$ structure $\mathcal{N}$ and refer to $\mathcal{N}$ as the pullback of $\mathcal{M}$.

If $f^{\prime}: \mathcal{S}_{1} \rightarrow \mathcal{S}_{2}$ is a second 1-morphism and $\iota: f \rightarrow f^{\prime}$ is a 2-isomorphism, then $\iota$ induces an isomorphism $\sigma: f^{-1} \mathcal{M} \rightarrow f^{\prime-1} \mathcal{M}$ such that

$$
\left(\theta_{f^{\prime}} \circ f^{\prime-1}(\alpha)\right) \circ \sigma=\theta_{f} \circ f^{-1}(\alpha)
$$

and hence an isomorphism of $\log$ structures $\iota(\mathcal{M}): f^{*} \mathcal{M} \rightarrow f^{\prime *} \mathcal{M}$.

DEFINITION 5.5. - A 1-morphism $\left(\mathcal{S}_{1}, \mathcal{M}_{\mathcal{S}_{1}}\right) \rightarrow\left(\mathcal{S}_{2}, \mathcal{M}_{\mathcal{S}_{2}}\right)$ between log algebraic stacks is a pair $\left(f, f^{b}\right)$, where $f: \mathcal{S}_{1} \rightarrow \mathcal{S}_{2}$ is a 1-morphism of stacks and $f^{b}: f^{*} \mathcal{M}_{\mathcal{S}_{2}} \rightarrow \mathcal{M}_{\mathcal{S}_{1}}$ is 
a morphism of $\log$ structures on $\mathcal{S}_{1}$. A 2-isomorphism $\left(f, f^{b}\right) \rightarrow\left(f^{\prime}, f^{\prime b}\right)$ is a 2-isomorphism $\iota: f \rightarrow f^{\prime}$ such that $f^{\prime b} \circ \iota\left(\mathcal{M}_{\mathcal{S}_{1}}\right)=f^{b}$.

The following proposition gives a useful description of fine log structures in terms of a covering:

Proposition 5.6. - Let $\mathcal{S}$ be an algebraic stack, and let $z: Z \rightarrow \mathcal{S}$ be a morphism from a scheme which is flat, surjective, and locally of finite presentation. Then there is a natural equivalence of categories between the category of fine log structures on $\mathcal{S}_{\text {lis-et }}$ and the category of pairs $(\mathcal{M}, \sigma)$, where $\mathcal{M}$ is a fine log structure on $Z$ and $\sigma: \operatorname{pr}_{1}^{*} \mathcal{M} \rightarrow \operatorname{pr}_{2}^{*} \mathcal{M}$ is an isomorphism of $\log$ structures on $Z \times{ }_{\mathcal{S}} Z$ such that

$$
\operatorname{pr}_{13}^{*}(\sigma)=\operatorname{pr}_{23}^{*}(\sigma) \circ \operatorname{pr}_{12}^{*}(\sigma)
$$

on $Z \times \times_{\mathcal{S}} Z \times_{\mathcal{S}} Z$.

Proof. - Given a fine $\log$ structure $\mathcal{N}$ on $\mathcal{S}$, we obtain a pair $(\mathcal{M}, \sigma)$ as in the proposition by setting $\mathcal{M}$ equal to $z^{*} \mathcal{N}$ and letting $\sigma$ be the isomorphism induced by the tautological isomorphism of functors $z \circ \mathrm{pr}_{1} \simeq z \circ \mathrm{pr}_{2}$ on $Z \times_{\mathcal{S}} Z$. In this way we obtain a functor

$$
F:(\text { fine } \log \text { structures on } \mathcal{S}) \rightarrow(\text { category of pairs }(\mathcal{M}, \sigma))
$$

which we claim is an equivalence.

To show that $F$ is fully faithful, let $\mathcal{N}$ and $\mathcal{N}^{\prime}$ be fine $\log$ structures on $\mathcal{S}$ and consider the map

$$
\operatorname{Hom}\left(\mathcal{N}, \mathcal{N}^{\prime}\right) \rightarrow \operatorname{Hom}\left((\mathcal{M}, \sigma),\left(\mathcal{M}^{\prime}, \sigma^{\prime}\right)\right)
$$

where $(\mathcal{M}, \sigma)$ and $\left(\mathcal{M}^{\prime}, \sigma^{\prime}\right)$ are the images of $\mathcal{N}$ and $\mathcal{N}^{\prime}$ under $F$.

To see that (5.6.2) is injective, suppose $\varphi, \psi: \mathcal{N} \rightarrow \mathcal{N}^{\prime}$ are two maps for which $F(\varphi)=F(\psi)$. If $(V, v)$ is an object of Lis-Et $(\mathcal{S})$, we can find a 2-commutative diagram

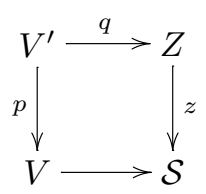

where $p: V^{\prime} \rightarrow V$ is an fppf cover, and by assumption $p^{*}\left(\varphi_{(V, v)}\right)=p^{*}\left(\psi_{(V, v)}\right)$, where $\varphi_{(V, v)}$ and $\psi_{(V, v)}$ denote the maps $\mathcal{N}_{(V, v)} \rightarrow \mathcal{N}_{(V, v)}^{\prime}$ induced by $\varphi$ and $\psi$. But then by (A.5), $\varphi_{(V, v)}=\psi_{(V, v)}$, and hence (5.6.2) is injective. Note that in the case when $(Z, z)$ is a smooth cover we can by ([4], IV.17.16.3) find a diagram (5.6.3) with $p: V^{\prime} \rightarrow V$ an étale cover and so (A.5) is not needed in this case.

Conversely, suppose given a morphism $\varepsilon:(\mathcal{M}, \sigma) \rightarrow\left(\mathcal{M}^{\prime}, \sigma^{\prime}\right)$. Then for every $(V, v) \in$ $\operatorname{Lis-\operatorname {Et}}(\mathcal{S})$ we can find a diagram as in (5.6.3) and $q^{*}(\varepsilon)$ defines a morphism

$$
\rho: p^{*} \mathcal{N}_{(V, v)} \rightarrow p^{*} \mathcal{N}_{(V, v)}^{\prime} .
$$

Moreover, the fact that $\varepsilon$ is compatible with $\sigma$ and $\sigma^{\prime}$ implies that if $h: V^{\prime} \times_{V} V^{\prime} \rightarrow V$ denotes the map $\operatorname{pr}_{1} \circ p=\operatorname{pr}_{2} \circ p$, then the two maps

$$
\operatorname{pr}_{1}^{*}(\rho), \operatorname{pr}_{2}^{*}(\rho): h^{*} \mathcal{N}_{(V, v)} \rightarrow h^{*} \mathcal{N}_{(V, v)}^{\prime}
$$


are equal. Then by (A.5) we obtain a morphism $\psi_{(V, v)}: \mathcal{N}_{(V, v)} \rightarrow \mathcal{N}_{(V, v)}^{\prime}$, and the collection of maps $\left\{\psi_{(V, v)}\right\}$ defines a map $\mathcal{N} \rightarrow \mathcal{N}^{\prime}$ inducing $\varepsilon$. As before, in the case when $(Z, z)$ is a smooth cover we can find a diagram as in (5.6.3) with $V^{\prime} / V$ an étale cover so (A.5) is not needed. Thus (5.6.2) is bijective.

To complete the proof of the proposition, it remains only to see that any pair $(\mathcal{M}, \sigma)$ is induced by a fine $\log$ structure $\mathcal{N}$ on $\mathcal{S}$. For this, let $(V, v) \in \operatorname{Lis-\operatorname {Et}}(\mathcal{S})$ and choose a diagram as in (5.6.3). The pair $(\mathcal{M}, \sigma)$ define a $\log$ structure $q^{*} \mathcal{M}$ on $V^{\prime}$ together with an isomorphism $\iota: \operatorname{pr}_{1}^{*} q^{*} \mathcal{M} \simeq \operatorname{pr}_{2}^{*} q^{*} \mathcal{M}$ on $V^{\prime} \times_{V} V^{\prime}$ which satisfies the cocycle condition on $V^{\prime} \times_{V} V^{\prime} \times_{V} V^{\prime}$ because $\sigma$ satisfies the cocycle condition. Hence by (A.5) we obtain a unique log structure $\mathcal{N}_{(V, v)}$ on $V$ inducing the pair $\left(q^{*} \mathcal{M}, \iota\right)$ on $V^{\prime}$ (as above when $Z / \mathcal{S}$ is smooth (A.5) is not needed since we can choose $V^{\prime} / V$ étale). The family $\left\{\mathcal{N}_{(V, v)}\right\}$ comes with natural transition maps $\psi_{(V, v)}$, and hence we obtain a fine $\log$ structure $\mathcal{N}$ on $\mathcal{S}$ which induces the pair $(\mathcal{M}, \sigma)$.

Remark 5.7. - As pointed out above, the proof of (5.6) does not require the use of result from the appendix in the case when $z: Z \rightarrow \mathcal{S}$ is smooth.

COROLlary 5.8. - Suppose $S$ is a fine log scheme, and let $\mathcal{S}$ be an algebraic stack. Then the groupoid of morphisms of stacks $\mathcal{S} \rightarrow \mathcal{L} \operatorname{og}_{S}([14], 2.2)$ is naturally equivalent to the groupoid of pairs $(\mathcal{M}, f)$, where $\mathcal{M}$ is a fine log structure on $\mathcal{S}$ and $f:(\mathcal{S}, \mathcal{M}) \rightarrow S$ is a morphism of log stacks.

Proof. - Let $U \rightarrow \mathcal{S}$ be a smooth cover. By ([14], 3.2), the category of morphisms $\mathcal{S} \rightarrow \mathcal{L} \operatorname{og}_{S}$ is equivalent to the category of triples $\left(\mathcal{M}_{U}, f_{U}, \sigma\right)$, where $\mathcal{M}_{U}$ is a fine log structure on $U, f_{U}:\left(U, \mathcal{M}_{U}\right) \rightarrow S$ is a morphism of fine log schemes, and $\sigma: \operatorname{pr}_{1}^{*} \mathcal{M}_{U} \rightarrow \operatorname{pr}_{2}^{*} \mathcal{M}_{U}$ is an isomorphism of log structures on $U \times{ }_{\mathcal{S}} U$ satisfying the cocycle condition on $U \times{ }_{\mathcal{S}} U \times{ }_{\mathcal{S}} U$ for which the two maps

$$
\left(h, \operatorname{pr}_{1}^{*}\left(f^{b}\right)\right),\left(h, \sigma^{-1} \circ \operatorname{pr}_{2}\left(f^{b}\right)\right):\left(U \times_{\mathcal{S}} U, \operatorname{pr}_{1}^{*} \mathcal{M}\right) \rightarrow S
$$

are equal. By (5.6), the category of such triples $\left(\mathcal{M}_{U}, f_{U}, \sigma\right)$ is equivalent to the category of pairs $(\mathcal{M}, f)$ as in the corollary.

If $(\mathcal{S}, \mathcal{M})$ is a fine log algebraic stack, we can define a fibered category $\log _{\left(\mathcal{S}, \mathcal{M}_{\mathcal{S}}\right)}$ just as we did in the case when $\mathcal{S}$ is a scheme. The objects of $\mathcal{L}_{\log }\left(\mathcal{S}, \mathcal{M}_{\mathcal{S}}\right)$ are pairs $(T, t)$, where $T$ is a fine $\log$ scheme and $t: T \rightarrow\left(\mathcal{S}, \mathcal{M}_{\mathcal{S}}\right)$ is a 1-morphism between log algebraic stacks. A morphism $\left(T^{\prime}, t^{\prime}\right) \rightarrow(T, t)$ is a pair $(\sigma, \iota)$, where $\sigma: T^{\prime} \rightarrow T$ is a strict morphism of log schemes, and $\iota: t^{\prime} \simeq t \circ \sigma$ is a 2 -isomorphism between morphism of log algebraic stacks. The natural functor $(T, t) \mapsto \stackrel{\circ}{T}$ makes $\mathcal{L}_{\log }\left(\mathcal{S}, \mathcal{M}_{\mathcal{S}}\right)$ a fibered category over the category of schemes. Note that there is a natural morphism $\mathcal{L} \operatorname{og}_{\left(\mathcal{S}, \mathcal{M}_{\mathcal{S}}\right)} \rightarrow \mathcal{S}$ obtained by sending an object $(T, t)$ to the 1-morphism $\stackrel{\circ}{T} \rightarrow \mathcal{S}$ underlying $t$.

PROPOSITION 5.9. - The fibered category $\log _{\left(\mathcal{S}, \mathcal{M}_{\mathcal{S}}\right)}$ is an algebraic stack locally of finite presentation over $\mathcal{S}$.

Proof. - It follows from the fact that $\mathcal{S}$ is a stack that $\log _{\left(\mathcal{S}, \mathcal{M}_{\mathcal{S}}\right)}$ is also a stack with respect to the étale topology.

From this and (1.1) follows the case when $\mathcal{S}$ is an algebraic space. Indeed, both (1.2.4) and (1.2.5) can be verified after replacing $\mathcal{S}$ by an étale cover. Moreover, the statement that $\mathcal{L}_{\left(\mathcal{S}, M_{\mathcal{S}}\right)} / \mathcal{S}$ is locally of finite presentation can also be verified after replacing $\mathcal{S}$ by an étale cover.

To prove the proposition in the general case, we first verify (1.2.4) and (1.2.5). 
To show (1.2.4), suppose given a scheme $X$, two objects $x_{1}, x_{2} \in \mathcal{S}$ over $X$, and two fine $\log$ structures $\mathcal{M}_{1}$ and $\mathcal{M}_{2}$ on $X$ together with maps $x_{i}^{b}: x_{i}^{*} \mathcal{M}_{\mathcal{S}} \rightarrow \mathcal{M}_{i}(i=1,2)$, and define $I$ to be the functor on the category of $X$ schemes whose value on $f: Z \rightarrow X$ is the set of pairs $(\iota, \sigma)$, where $\iota: f^{*}\left(x_{1}\right) \rightarrow f^{*}\left(x_{2}\right)$ is an isomorphism in $\mathcal{S}$ over $Z$ and $\sigma: f^{*} \mathcal{M}_{1} \rightarrow f^{*} \mathcal{M}_{2}$ is an isomorphism of log structures such that the diagram

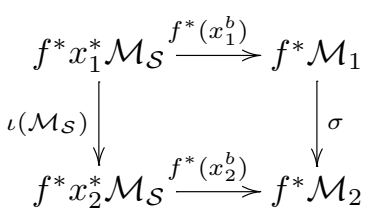

commutes. To show that the diagonal

$$
\Delta: \log _{\left(\mathcal{S}, \mathcal{M}_{\mathcal{S}}\right)} \rightarrow \mathcal{L}_{o_{\left(\mathcal{S}, \mathcal{M}_{\mathcal{S}}\right)}} \times \mathcal{L}_{\log _{\left(\mathcal{S}, \mathcal{M}_{\mathcal{S}}\right)}}
$$

is representable, it suffices to show that for any collection of data as above the functor $I$ is representable by an algebraic space of finite presentation over $X$. Let $\pi: X^{\prime} \rightarrow X$ be the fiber product $X \times{ }_{\left(x_{1}, x_{2}\right), \mathcal{S} \times \mathcal{S}, \Delta} \mathcal{S}$. Since $\mathcal{S}$ is algebraic, $X^{\prime}$ is representable by an algebraic space of finite presentation over $X$. It follows from the definition of $I$ that $I$ is naturally isomorphic to the fiber product of the diagram

$$
\log _{\left(X^{\prime}, \pi^{*} x_{1}^{*} \mathcal{M}_{\mathcal{S}}\right) \stackrel{\Delta}{\longrightarrow} \mathcal{L}_{\log _{\left(X^{\prime}, \pi^{*} x_{1}^{*} \mathcal{M}_{\mathcal{S}}\right)}} \times^{\downarrow_{X}}\left(\left(\mathrm{id}, \pi^{*}\left(x_{1}^{b}\right)\right),\left(\mathrm{id}, \pi^{*}\left(x_{2}^{b}\right) \circ \iota\right)\right)}^{X_{\left(X^{\prime}, \pi^{*} x_{1}^{*} \mathcal{M}_{\mathcal{S}}\right)}^{\prime},}
$$

where $\iota$ denotes the universal isomorphism $\iota: \pi^{*} x_{1}^{*} \mathcal{M}_{\mathcal{S}} \simeq \pi^{*} x_{2}^{*} \mathcal{M}_{\mathcal{S}}$ over $X^{\prime}$. Hence by the case when $\mathcal{S}$ is an algebraic space, the functor $I$ is representable by an algebraic space of finite presentation over $X^{\prime}$. Since $X^{\prime} / X$ is of finite presentation, $I / X$ is also of finite presentation.

To construct a smooth cover $U \rightarrow \mathcal{L}_{o} g_{\left(\mathcal{S}, \mathcal{M}_{\mathcal{S}}\right)}$ as in (1.2.5), we may replace $\mathcal{S}$ by a smooth cover, and hence the existence of such a $U$ follows from (1.1).

Finally, to prove that $\mathcal{L}_{\left(\mathcal{S}, \mathcal{M}_{\mathcal{S}}\right)}$ is locally of finite presentation over $\mathcal{S}$, we may again replace $\mathcal{S}$ by a smooth cover by ([14], 4.14), and hence this also follows from (1.1).

Note that as in the case of schemes (3.19), if $\left(\mathcal{S}, \mathcal{M}_{\mathcal{S}}\right)$ is a log algebraic stack, then there is a natural map

$$
s: \mathcal{S} \rightarrow \mathcal{L}_{\left(\mathcal{S}, \mathcal{M}_{\mathcal{S}}\right)}
$$

obtained from the functor

$$
(t: T \rightarrow \mathcal{S}) \mapsto\left((t, \mathrm{id}):\left(T, t^{*} \mathcal{M}_{\mathcal{S}}\right) \rightarrow\left(\mathcal{S}, \mathcal{M}_{\mathcal{S}}\right)\right)
$$

It follows from (3.19(ii)) that $s$ is an open immersion since this can be verified after replacing $\mathcal{S}$ by a smooth cover.

Observe also that if

$$
f:\left(\mathcal{S}_{1}, \mathcal{M}_{\mathcal{S}_{1}}\right) \rightarrow\left(\mathcal{S}_{2}, \mathcal{M}_{\mathcal{S}_{2}}\right)
$$

$4^{\mathrm{e}}$ SÉRIE - TOME $36-2003-\mathrm{N}^{\circ} 5$ 
is a 1-morphism between log algebraic stacks, then there is a morphism

$$
\mathcal{L} o g(f): \mathcal{L}_{\left(\mathcal{S}_{1}, \mathcal{M}_{\mathcal{S}_{1}}\right)} \rightarrow \mathcal{L}_{o g}\left(\mathcal{S}_{2}, \mathcal{M}_{\mathcal{S}_{2}}\right)
$$

obtained from

$$
\left(g: T \rightarrow\left(\mathcal{S}_{1}, \mathcal{M}_{\mathcal{S}_{1}}\right)\right) \mapsto\left(g \circ f: T \rightarrow\left(\mathcal{S}_{2}, \mathcal{M}_{\mathcal{S}_{2}}\right)\right) .
$$

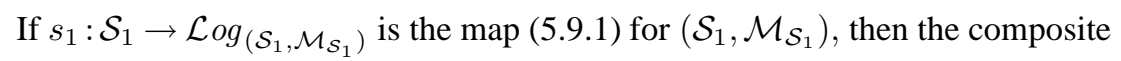

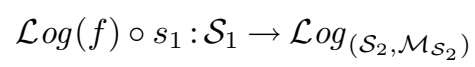

is the morphism induced by the composite

$$
\left(t: T \rightarrow \mathcal{S}_{1}\right) \mapsto\left(\left(t, t^{*}\left(f^{b}\right)\right):\left(T, t^{*} \mathcal{M}_{\mathcal{S}_{1}}\right) \rightarrow\left(\mathcal{S}_{2}, \mathcal{M}_{\mathcal{S}_{2}}\right)\right) .
$$

Though we will only use the notions of $\mathcal{L}$ og representable and $\mathcal{L}$ og étale morphisms in what follows, we include the following two general definitions ((5.10)-(5.11)).

DEFINITION 5.10. - Let $\mathcal{P}$ be a property of morphism between algebraic stacks. We say that a morphism $f$ as in (5.9.2) has property $\log (\mathcal{P})$ if the morphism of algebraic stacks (5.9.3) has property $\mathcal{P}$, and that $f$ has property weak $\mathcal{L} o g(P)$ if (5.9.4) has property $\mathcal{P}$.

For example, the property $\mathcal{P}$ could be the property of being representable, smooth, or flat, in which case we sometimes say that $f$ is (weakly) $\mathcal{L}$ og representable, (weakly) $\mathcal{L}$ og smooth, and (weakly) $\mathcal{L}$ og flat.

For $\mathcal{L}$ og representable morphisms, one can define more properties (note that since (5.9.1) is an open immersion, $\mathcal{L}$ og representable implies weakly $\mathcal{L}$ og representable):

DEFINITION 5.11. - Let $\mathcal{P}$ be a property of representable morphisms of algebraic stacks, and let $f$ be a morphism of log algebraic stacks as in (5.9.2) which is $\mathcal{L} o g$ representable. We say that $f$ has property $\log (\mathcal{P})$ if the induced map (5.9.3) has property $\mathcal{P}$, and that $f$ has property weak $\mathcal{L} \operatorname{og}(\mathcal{P})$ if the map (5.9.4) has property $\mathcal{P}$.

For example, $\mathcal{P}$ could be the property of being étale, unramified, or quasi-finite. Below we shall see interesting examples ((5.23)-(5.29)) of $\mathcal{L}$ og representable morphisms whose underlying morphism of algebraic stacks is not representable.

The above definitions and results is the basic foundational work on log structures on stacks that we need, and we now turn our attention to "toric stacks".

Let $S$ be a scheme, and recall that for any fine monoid $P$, we have a $S$-scheme $S[P]=$ $\operatorname{Spec}\left(\mathcal{O}_{S}[P]\right)$ (1.2.1). For any affine scheme $\operatorname{Spec}(R)$ over $S$, there is a natural bijection

$$
S[P](\operatorname{Spec}(R))=\operatorname{Hom}_{\mathrm{Mon}}(P, R)
$$

which gives $S[P]$ the structure of a monoid scheme over $S$. There is a natural inclusion of monoid schemes $S\left[P^{g p}\right] \hookrightarrow S[P]$, and so the group scheme $S\left[P^{g p}\right]$ acts on $S[P]$ by translation. We denote by $\mathcal{S}_{P}$ the stack theoretic quotient of $S[P]$ by the action of $S\left[P^{g p}\right]$ (denoted $\left[S[P] / S\left[P^{g p}\right]\right]$ in $\left.([14], 4.6 .1)\right)$.

Remark 5.12. - When $P$ is a saturated and torsion free monoid, $S[P]$ with the action of $S\left[P^{g p}\right]$ is a toric variety. In this case $\mathcal{S}_{P}$ is a toric stack in the sense of L. Lafforgue ([13], IV.1.a). 
Example 5.13. - In the case when $P=\mathbb{N}^{r}$ for some $r \in \mathbb{N}, \mathcal{S}_{P}=\left[\mathbb{A}^{r} / \mathbb{G}_{m}^{r}\right]$. To give a 1-morphism $T \rightarrow \mathcal{S}_{P}$ is equivalent to giving $r$-line bundles $\mathcal{L}_{i}$ on $T$, together with morphisms of line bundles $s_{i}: \mathcal{L}_{i} \rightarrow \mathcal{O}_{T}$. Indeed the stack classifying such collections of data $\left(\mathcal{L}_{i}, s_{i}\right)_{i=1}^{r}$ is naturally equivalent to the stack associated to the prestack whose objects over a scheme $T$ are $r$-tuples of elements $\left(x_{1}, \ldots, x_{r}\right)$ in $\Gamma\left(T, \mathcal{O}_{T}\right)$, and whose morphisms $\left(x_{1}, \ldots, x_{r}\right) \rightarrow\left(x_{1}^{\prime}, \ldots, x_{r}^{\prime}\right)$ are $r$-tuples of elements $u_{i} \in \Gamma\left(T, \mathcal{O}_{T}^{*}\right)$ such that $x_{i}^{\prime}=u_{i} x_{i}$. This prestack is in turn naturally equivalent to that defined by $\mathbb{A}^{r}$ with its $\mathbb{G}_{m}^{r}$-action. Now such a collection $\left(\mathcal{L}_{i}, s_{i}\right)$ is what $\mathrm{K}$. Kato calls a Deligne-Faltings log structure in ([9], complement 1). Thus by (loc. cit.), $\mathcal{S}_{\mathbb{N}^{r}}$ is the classifying stack of pairs $(\mathcal{M}, \gamma)$, where $\mathcal{M}$ is a log structure and $\gamma: \mathbb{N}^{r} \rightarrow \overline{\mathcal{M}}$ is a map which étale locally lifts to a chart. Propositions (5.14) and (5.20) below generalize this example.

The action of $S\left[P^{g p}\right]$ on $S[P]$ extends naturally to an action on the log structure on $S[P]$ associated to the map $P \rightarrow \mathcal{O}_{S}[P]$. This just means that for a ring $R$ and maps $a: P \rightarrow R$ and $b: P^{g p} \rightarrow R^{*}$, there is a natural isomorphism between the log structure associated to the map $a$ and that associated to $a \cdot b$. Such an isomorphism is provided by the map

$$
P \rightarrow R^{*} \oplus P, \quad p \mapsto\left(b(p)^{-1}, p\right) .
$$

It follows from this and (5.6) that the log structure on $S[P]$ descends to a log structure $\mathcal{M}_{\mathcal{S}_{P}}$ on $\mathcal{S}_{P}$. Note that in the case when $P^{g p}$ is torsion free, $S[P]$ is smooth over $\mathcal{S}_{P}$ so Remark (5.7) applies in this case. Note also that there is a natural map $\pi_{P}: P \rightarrow \overline{\mathcal{M}}_{\mathcal{S}_{P}}$.

If $t: T \rightarrow \mathcal{S}_{P}$ is any 1-morphism from a scheme, we obtain a pair $\left(t^{*} \mathcal{M}_{\mathcal{S}_{P}}, t^{*} \pi_{P}\right)$, where $t^{*} \mathcal{M}_{\mathcal{S}_{P}}$ is a fine $\log$ structure on $T$ and $t^{*} \pi_{P}: P \rightarrow \bar{t}^{*} \mathcal{M}_{\mathcal{S}_{P}}$ is a morphism of sheaves of monoids. Moreover, since $S[P] \rightarrow \mathcal{S}_{P}$ is flat and surjective, the map $t^{*} \pi_{P}$ lifts fppf locally to a chart for $t^{*} \mathcal{M}_{\mathcal{S}_{P}}$. In the case when $P$ is saturated, the map $S[P] \rightarrow \mathcal{S}_{P}$ is smooth, and then the existence of a quasi-section for smooth morphisms ([4], IV.17.16.3) implies that the map $t^{*} \pi_{P}$ lifts étale locally on $T$ to a chart for $t^{*} \mathcal{M}_{\mathcal{S}_{P}}$.

Proposition 5.14. - Let $P$ be a fine monoid. Then the pair $\left(\mathcal{M}_{\mathcal{S}_{P}}, \pi_{P}\right)$ on $\mathcal{S}_{P}$ induces an equivalence of stacks between $\mathcal{S}_{P}$ and the fibered category $\mathcal{S}_{P}^{\prime}$ over the category of $S$-schemes whose fiber over a scheme $T$ is the groupoid of pairs $(\mathcal{N}, \gamma)$, where $\mathcal{N}$ is a fine log structure on $T$ and $\gamma: P \rightarrow \overline{\mathcal{N}}$ is a morphism which locally in the fppf topology on T lifts to a chart for $\mathcal{N}$.

Remark 5.15. - When $P$ is fs, (2.4(ii)) shows that $\mathcal{S}_{P}^{\prime}$ is equivalent to the fibered category classifying pairs $(\mathcal{N}, \gamma)$, where $\mathcal{N}$ is a fs $\log$ structure and $\gamma: P \rightarrow \overline{\mathcal{N}}$ is a morphism which locally in the étale topology lifts to a chart.

Remark 5.16. - The proof of (5.14) given below uses the result, proven in the appendix (A.3), that $\mathcal{S}_{P}^{\prime}$ is a stack with respect to the fppf topology. However, as pointed out in the proof, when $P$ is fs and torsion free one needs only that $\mathcal{S}_{P}^{\prime}$ is a stack with respect to the étale topology, and (A.3) is not needed.

Proof of (5.14). - Let

$$
F: \mathcal{S}_{P} \rightarrow \mathcal{S}_{P}^{\prime}
$$

denote the morphism of fibered categories ([14], 2.2) defined by $\left(\mathcal{M}_{\mathcal{S}_{P}}, \pi_{P}\right)$. Note that $\mathcal{S}_{P}^{\prime}$ is a stack with respect to the étale topology since étale sheaves and morphisms between étale sheaves may be constructed étale locally, and in fact $\mathcal{S}_{P}^{\prime}$ is a stack with respect to the fppf topology by (A.3).

Since $\mathcal{S}_{P}$ is also a stack with the respect to the fppf topology ([14], 10.7(a)), it suffices to show that $F$ is fully faithful and that every object of $\mathcal{S}_{P}^{\prime}$ is fppf locally isomorphic to an object in the 
image of $F$. In the case when $P$ is fs, the proof given below shows that $F$ is fully faithful and that every object of $\mathcal{S}_{P}^{\prime}$ is étale locally in the image of $F$, so in this case one only needs to know that $\mathcal{S}_{P}^{\prime}$ is a stack with respect to the étale topology and so (A.3) is not needed.

To prove the full faithfulness, it suffices to show that for any affine $S$-scheme $\operatorname{Spec}(R)$, and points $a, b \in S[P](R)$ inducing objects $\left(\mathcal{N}_{a}, \gamma_{a}\right)$ and $\left(\mathcal{N}_{b}, \gamma_{b}\right)$ of $\mathcal{S}_{P}^{\prime}(R)$, any isomorphism $\sigma$ between $\left(\mathcal{N}_{b}, \gamma_{b}\right)$ and $\left(\mathcal{N}_{a}, \gamma_{a}\right)$ is induced by a unique point $u \in\left(S[P] \times_{S} S\left[P^{g p}\right]\right)(R)$ such that $\operatorname{pr}_{1}(u)=a$ and $\operatorname{pr}_{2}(u)=b$ (where $\operatorname{pr}_{1}$ is the projection and $\operatorname{pr}_{2}$ is given by the action of $S\left[P^{g p}\right]$ on $S[P]$ ). Now the isomorphism $\sigma$ is by definition an isomorphism $\sigma: \mathcal{N}_{b} \simeq \mathcal{N}_{a}$ such that $\bar{\sigma} \circ \gamma_{b}=\gamma_{a}$. For such an isomorphism, there exists for each $p \in P$, a unique unit $u_{p} \in R^{*}$ such that $\lambda\left(u_{p}\right)+p=\sigma(p)$ in $\mathcal{N}_{a}$. In other words, there exists a unique point $u \in S\left[P^{g p}\right](R)$ such that $\sigma$ is induced from the map

$$
P \rightarrow R^{*} \oplus P, \quad p \mapsto\left(u_{p}, p\right),
$$

and the fact that $\sigma$ is an isomorphism of $\log$ structures implies that $\operatorname{pr}_{1}(u)=a$ and $\operatorname{pr}_{2}(u)=b$. Thus $F$ is fully faithful.

Now by definition any object $(\mathcal{N}, \gamma)$ of $\mathcal{S}_{P}^{\prime}$ over some $S$-scheme $T$, is fppf locally (étale locally when $P$ is fs by (2.4(ii))) obtained from a map $P \rightarrow \Gamma\left(T, \mathcal{O}_{T}\right)$ since $\gamma$ is assumed to lift to a chart fppf locally. This implies that every object of $\mathcal{S}_{P}^{\prime}$ is fppf locally (étale locally when $P$ is fs) obtained from a point of $S[P]$ and hence is in the image of $F$.

It is also interesting to consider the fibered category $\mathcal{S}_{P}^{\log }$ over the category of fine log schemes defined by $\mathcal{S}_{P}$ with its $\log$ structure.

In general, if $\left(\mathcal{S}, \mathcal{M}_{\mathcal{S}}\right)$ is an algebraic stack with a fine log structure, we obtain a stack denoted $\left(\mathcal{S}, \mathcal{M}_{\mathcal{S}}\right)^{\log }$ (or simply $\mathcal{S}^{\log }$ if no confusion seems likely to arise) over the category of fine $\log$ schemes. The objects of this stack are pairs $(T, t)$, where $T$ is a fine log scheme and $t: T \rightarrow\left(\mathcal{S}, \mathcal{M}_{\mathcal{S}}\right)$ is a 1 -morphism $t: T \rightarrow\left(\mathcal{S}, \mathcal{M}_{\mathcal{S}}\right)$ between log algebraic stacks (5.5). A morphism $(T, t) \rightarrow\left(T^{\prime}, t^{\prime}\right)$ is a pair $(\varphi, \iota)$, where $\varphi: T \rightarrow T^{\prime}$ is a morphism of log schemes and $\iota: t \rightarrow t^{\prime} \circ \varphi$ is a 2-isomorphism. If we give the category of fine log schemes the topology in which covers are given by strict étale morphisms (the strict étale topology), then $\mathcal{S}^{\log }$ is in fact a stack.

In the case of $\mathcal{S}_{P}$, the associated stack $\mathcal{S}_{P}^{\log }$ "is" (i.e. is equivalent to) a functor. Indeed to give a 1-morphism $t: \stackrel{\circ}{T} \rightarrow \mathcal{S}_{P}$ is by (5.14) equivalent to giving a log structure $\mathcal{M}$ on $T$ together with a map $P \rightarrow \overline{\mathcal{M}}$ which fppf locally (étale locally in the fs case) lifts to a chart. Now if we are given a morphism $\iota: \mathcal{M} \rightarrow \mathcal{M}_{T}$ of $\log$ structures, the fact that $\iota$ is a bijection on units implies that there are no non-trivial automorphisms $\sigma$ of $\mathcal{M}$ which induce the identity on $\overline{\mathcal{M}}$ for which $\iota=\iota \circ \sigma$. Hence the objects of $\mathcal{S}_{P}^{\log }$ admit no non-trivial automorphisms, and so $\mathcal{S}_{P}^{\log }$ is equivalent to a functor. This functor has the following interpretation:

PROPOSITION 5.17. - The functor $\mathcal{S}_{P}^{\log }$ is naturally isomorphic to the functor

$$
\underline{\operatorname{Hom}}(P, \overline{\mathcal{M}}): T \mapsto \operatorname{Hom}\left(P, \Gamma\left(T, \overline{\mathcal{M}}_{T}\right)\right) .
$$

Moreover, a morphism $T \rightarrow\left(\mathcal{S}_{P}, \mathcal{M}_{\mathcal{S}_{P}}\right)$ is strict if and only if the corresponding map $\pi: P \rightarrow \overline{\mathcal{M}}_{T}$ lifts fppf locally to a chart.

Remark 5.18. - When $P$ is fs, the condition that $\pi: P \rightarrow \overline{\mathcal{M}}_{T}$ lifts fppf locally to a chart is by (2.4(ii)) equivalent to the condition that $\pi$ lifts étale locally to a chart.

Remark 5.19. - In the proof of (5.17) given below, we use a result from the appendix (A.4) when asserting that the map (5.19.1) is a map between sheaves for the strict fppf topology on 
the category of fine $\log$ schemes. If $P$ is saturated and torsion free and if one restricts $\mathcal{S}_{P}^{\log }$ and $\underline{\operatorname{Hom}}(P, \overline{\mathcal{M}})$ to the category of fs log schemes, then one needs only that the map (5.19.1) is a map of sheaves with respect to the strict étale topology and the use of (A.4) can be avoided.

Proof of (5.17). - Let $(\mathcal{N}, \pi, \iota) \in \mathcal{S}_{P}^{\log }(T)$ be an object over some log scheme $T$, where $\mathcal{N}$ is a fine $\log$ structure on $T, \pi: P \rightarrow \overline{\mathcal{N}}$ is a morphism which fppf locally lifts to a chart, and $\iota: \mathcal{N} \rightarrow \mathcal{M}_{T}$ is a morphism of $\log$ structures. Then $\bar{\iota} \circ \pi: P \rightarrow \overline{\mathcal{M}}_{T}$ determines an element of $\underline{\operatorname{Hom}}(P, \overline{\mathcal{M}})(T)$ and this defines a map

$$
M: \mathcal{S}_{P}^{\log } \rightarrow \underline{\operatorname{Hom}}(P, \overline{\mathcal{M}}) .
$$

It follows from (A.4) and ([14], 10.7(a)) that $\mathcal{S}_{P}^{\log }$ is a sheaf for the strict fppf topology on the category of $\log$ schemes. Here by the strict fppf topology we mean the topology on the category of $\log$ schemes locally of finite presentation over $S$ where coverings are given by strict morphisms whose underlying morphism of schemes is faithfully flat and locally of finite presentation. Moreover, by ([3], VIII.9.1) the functor $\underline{\operatorname{Hom}}(P, \overline{\mathcal{M}})$ is also a sheaf with respect to the strict fppf topology. Hence to show that (5.19.1) is an isomorphism it suffices to show the following:

(5.19.2) Any section of $\underline{\operatorname{Hom}}(P, \overline{\mathcal{M}})$ is fppf locally in the image of $M$;

(5.19.3) Any two sections of $\mathcal{S}_{P}^{\log }$ which map to the same element under $M$ are fppf locally isomorphic.

Statement (5.19.2) follows from (2.3) which shows that any map $P \rightarrow \overline{\mathcal{M}}_{T}$ on some log scheme $T$ is fppf locally (étale locally when $\mathcal{M}$ is fs) on $\stackrel{\circ}{T}$ induced by a morphism of log schemes $T \rightarrow \operatorname{Spec}(P \rightarrow \mathbb{Z}[P])$.

To prove statement (5.19.3), we show that if $T$ is a log scheme then two elements $(\mathcal{N}, \pi, \iota)$ and $\left(\mathcal{N}^{\prime}, \pi^{\prime}, \iota^{\prime}\right)$ of $\mathcal{S}_{P}^{\log }(T)$ are isomorphic if and only if the induced maps

$$
\bar{\iota} \circ \pi, \bar{\iota}^{\prime} \circ \pi^{\prime}: P \rightarrow \overline{\mathcal{M}}_{T}
$$

are equal. The "only if" direction is clear. For the other direction, observe that by the uniqueness of isomorphisms it suffices to construct an isomorphism fppf locally. Thus by (2.3) we may assume we have liftings $\tilde{\pi}$ and $\tilde{\pi}^{\prime}$ of $\pi$ and $\pi^{\prime}$ (note that when $\mathcal{N}$ and $\mathcal{N}^{\prime}$ are fs we have such liftings étale locally). In this case, for every $p \in P$ there exists a unique unit $u_{p} \in \mathcal{O}_{T}^{*}$ such that $\iota(\tilde{\pi}(p))=\lambda\left(u_{p}\right)+\iota^{\prime}\left(\tilde{\pi}^{\prime}(p)\right)$. The desired isomorphism $\mathcal{N} \simeq \mathcal{N}^{\prime}$ is then obtained from the isomorphism induced by the map

$$
P \rightarrow \mathcal{O}_{T}^{*} \oplus P, \quad p \mapsto\left(u_{p}, p\right) .
$$

From this it follows that (5.19.1) is an isomorphism.

Finally we show that a morphism $f: T \rightarrow\left(\mathcal{S}_{P}, \mathcal{M}_{\mathcal{S}_{P}}\right)$ is strict if and only if $P \rightarrow \overline{\mathcal{M}}_{T}$ lifts fppf locally to a chart. The "only if" direction is clear. As for the "if" direction, suppose $f: T \rightarrow\left(\mathcal{S}_{P}, \mathcal{M}_{\mathcal{S}_{P}}\right)$ is such that $P \rightarrow \overline{\mathcal{M}}_{T}$ fppf locally lifts to a chart. Locally in the fppf topology on $\stackrel{\circ}{T}$, we can lift $f^{*}\left(\pi_{P}\right): P \rightarrow f^{-1} \mathcal{M}_{\mathcal{S}_{P}}$ to a chart $\beta: P \rightarrow f^{*} \mathcal{M}_{\mathcal{S}_{P}}$, and by (2.4(i)) the composite $f^{b} \circ \beta: P \rightarrow \mathcal{M}_{T}$ is also a chart. Hence $f^{b}: f^{*} \mathcal{M}_{\mathcal{S}_{P}} \rightarrow \mathcal{M}_{T}$ is fppf-locally an isomorphism. Therefore, $\bar{f}^{b}: \bar{f}^{*} \mathcal{M}_{\mathcal{S}_{P}} \rightarrow \overline{\mathcal{M}}_{T}$ is an isomorphism (since this can be verified after pulling back by any surjective morphism $X^{\prime} \rightarrow X$ ) and so by ([8], 3.2) the map $f^{b}$ is an isomorphism as well.

The construction of $\mathcal{S}_{P}$ is functorial. More precisely, if $l: Q \rightarrow P$ is a morphism of monoids, then the natural map $S[P] \rightarrow S[Q]$ is compatible with the actions of $S\left[P^{g p}\right]$ and $S\left[Q^{g p}\right]$, and 
hence $l$ induces a map $\mathcal{S}(l): \mathcal{S}_{P} \rightarrow \mathcal{S}_{Q}$. Moreover, there is a natural morphism of log structures $\eta_{l}: \mathcal{S}(l)^{*} \mathcal{M}_{\mathcal{S}_{Q}} \rightarrow \mathcal{M}_{\mathcal{S}_{P}}$ for which the diagram

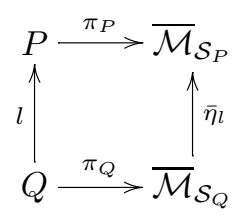

commutes.

We can describe this map $\mathcal{S}(l): \mathcal{S}_{P} \rightarrow \mathcal{S}_{Q}$ in terms of the modular interpretation of $\mathcal{S}_{P}$ given in Proposition (5.14). By (5.14), to give an object of $\mathcal{S}_{P}$ over some scheme $T$ is equivalent to giving a pair $(\mathcal{N}, \gamma)$, where $\mathcal{N}$ is a fine log structure on $T$ and $\gamma: P \rightarrow \overline{\mathcal{N}}$ is a morphism of sheaves of monoids which fppf locally lifts to a chart. We construct an object $\left(\mathcal{N}^{\prime}, \gamma^{\prime}\right)$ of $\mathcal{S}_{Q}$ as follows. Locally in the fppf topology we can choose a chart $\beta: P \rightarrow \mathcal{N}$ lifting $\gamma$ (if $P$ is fs then by (2.4(ii)) we can find such a lifting étale locally), and we define $\left(\mathcal{N}^{\prime}, \gamma^{\prime}\right)$ to be the log structure associated to the composite

$$
Q \rightarrow P \stackrel{\beta}{\rightarrow} \mathcal{N} \rightarrow \mathcal{O}_{T}
$$

together with the natural map $\gamma^{\prime}: Q \rightarrow \overline{\mathcal{N}}^{\prime}$. If $\beta_{1}, \beta_{2}$ are two liftings of $\gamma$, then for each $q$, there exists a unique unit $u(q)$ such that $\beta_{1}(q)=\lambda(u(q))+\beta_{2}(q)$. If $\rho_{i}: \mathcal{N}_{i}^{\prime} \rightarrow \mathcal{N}(i=1,2)$ are the two morphisms of $\log$ structures obtained from $\beta_{1}$ and $\beta_{2}$, then the $u(q)$ define an isomorphism

$$
\varepsilon: \mathcal{N}_{1}^{\prime} \rightarrow \mathcal{N}_{2}^{\prime}, \quad q \mapsto \lambda(u(q))+q
$$

such that $\rho_{1}=\rho_{2} \circ \varepsilon$ and such that the two maps

$$
\bar{\varepsilon} \circ \gamma_{1}^{\prime}, \gamma_{2}^{\prime}: Q \rightarrow \overline{\mathcal{N}}_{2}^{\prime}
$$

are equal. Thus we get an object of $\mathcal{S}_{Q}$. This is $\mathcal{S}(l)(\mathcal{N}, \gamma)$.

Proposition 5.20.- Suppose $S$ is a fine log scheme and let $\mathcal{S}(l): \mathcal{S}_{P} \rightarrow \mathcal{S}_{Q}$ be the morphism of stacks over $\stackrel{\circ}{S}$ obtained from $l: Q \rightarrow P$. Suppose further that a chart $\beta: Q \rightarrow \mathcal{M}_{S}$ is given inducing a map $\stackrel{\circ}{\rightarrow} \mathcal{S}_{Q}$. Then the pullback of the triple $\left(\mathcal{M}_{\mathcal{S}_{P}}, \eta_{l}, \pi_{P}\right)$ to the stack $\mathcal{S}_{P} \times \mathcal{S}_{Q} \stackrel{\circ}{S}$ defines an isomorphism of $\stackrel{\circ}{S}$-stacks between $\mathcal{S}_{P} \times \mathcal{S}_{Q} \stackrel{\circ}{S}$ and the stack $\mathcal{S}_{P / Q}^{\prime}$ whose fiber over $f: T \rightarrow \stackrel{\circ}{S}$ is the groupoid of triples $(\mathcal{N}, \eta, \gamma)$, where $\mathcal{N}$ is a fine log structure on $T, \eta: f^{*} \mathcal{M}_{S} \rightarrow \mathcal{N}$ is a morphism of log structures, and $\gamma: P \rightarrow \overline{\mathcal{N}}$ is a morphism, which fppf locally lifts to a chart, such that the diagram

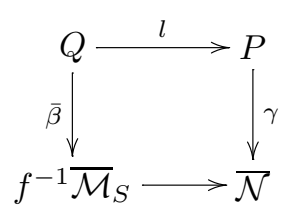

commutes.

Remark 5.21. - When $P$ is fs, (2.4(ii)) shows that $\mathcal{S}_{P / Q}^{\prime}$ is equivalent to the fibered category classifying triples $(\mathcal{N}, \eta, \gamma)$ as in the proposition where $\gamma$ lifts étale locally to a chart for $\mathcal{N}$. 
Proof of (5.20). - Let

$$
F: \mathcal{S}_{P} \times \mathcal{S}_{Q} \stackrel{\circ}{S} \rightarrow \mathcal{S}_{P / Q}^{\prime}
$$

be the functor induced by $\left(\mathcal{M}_{\mathcal{S}_{P}}, \eta_{l}, \pi_{P}\right)$.

That $F$ is fully faithful follows from the definition of the product of stacks $\mathcal{S}_{P} \times \mathcal{S}_{Q} \stackrel{\circ}{S}$.

To see that $F$ is essentially surjective, note that $F$ is a morphism of stacks with respect to the fppf topology by (A.3) and (A.4), and so it suffices to show that every object of $\mathcal{S}_{P / Q}^{\prime}$ is fppf locally in the image of $F$ (in the case when $P$ is fs the proof below actually shows that every object of $\mathcal{S}_{P / Q}^{\prime}$ is étale locally in the image of $F$ so one needs only that $\mathcal{S}_{P / Q}^{\prime}$ is a stack with respect to the étale topology which is immediate).

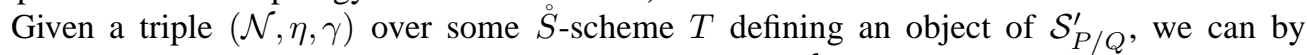
assumption fppf locally lift $\gamma$ to a chart $\tilde{\gamma}: P \rightarrow \mathcal{O}_{T}$ for $\mathcal{N}$ (when $P$ is fs we can by (2.4(ii)) find $\tilde{\gamma}$ étale locally on $T$ ). The induced diagram

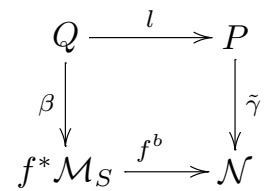

may not commute, but the commutativity of (5.20.1) insures that for each $q \in Q$ there exists a unique unit $u_{q} \in \mathcal{O}_{T}^{*}$ such that $\tilde{\gamma}(l(q))=\lambda\left(u_{q}\right)+f^{b}(\beta(q))$. If $\mathcal{M}$ denotes the log structure associated to $\tilde{\gamma} \circ l: Q \rightarrow \mathcal{N}$, then the map

$$
Q \rightarrow f^{*} \mathcal{M}_{S}, \quad q \mapsto \lambda\left(u_{q}\right)+\beta(q)
$$

defines an isomorphism between $\mathcal{M}$ and $f^{*} \mathcal{M}_{S}$ compatible with the maps to $\mathcal{N}$ and the maps $Q \rightarrow \overline{\mathcal{M}}$ and $Q \rightarrow f^{-1} \overline{\mathcal{M}}_{S}$. This implies that every triple $(\mathcal{N}, \eta, \gamma)$ is fppf locally obtained from a morphism to $\mathcal{S}_{P} \times \mathcal{S}_{Q} \stackrel{S}{S}$ and the proposition follows.

COROLLARY 5.22. - The fibered category over the category of S-log schemes defined by $\mathcal{S}_{P} \times \mathcal{S}_{Q} \stackrel{\circ}{S}$ is naturally equivalent to that defined by the functor which to any morphism of log schemes $f: T \rightarrow S$ associates the set of maps $\rho: P \rightarrow \Gamma\left(T, \overline{\mathcal{M}}_{T}\right)$ for which the diagram

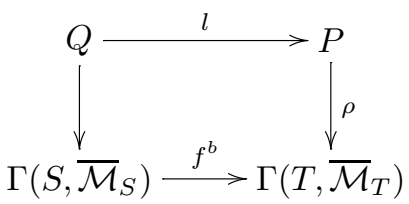

commutes.

Proof. - This follows from the same argument used in the proof of (5.17).

COROLlary 5.23. - For any morphism of fine monoids $l: Q \rightarrow P$, the induced morphism $\mathcal{S}(l):\left(\mathcal{S}_{P}, \mathcal{M}_{\mathcal{S}_{P}}\right) \rightarrow\left(\mathcal{S}_{Q}, \mathcal{M}_{\mathcal{S}_{Q}}\right)$ is $\mathcal{L}$ og representable and $\mathcal{L}$ og étale.

Proof. - We have to show that the map

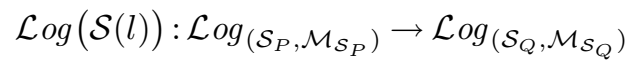


is representable and étale.

To show that $\log (\mathcal{S}(l))$ is representable it suffices by ([14], 8.1.2) to show that for any scheme $T$ and object $t \in \mathcal{L} \operatorname{og}\left(\mathcal{S}_{P}, \mathcal{M}_{\mathcal{S}_{P}}\right)(T)$ in the fiber over $T$, there are no non-trivial automorphisms of $t$ whose image under $\log (\mathcal{S}(l))$ is the identity. But if $T$ is a scheme, then

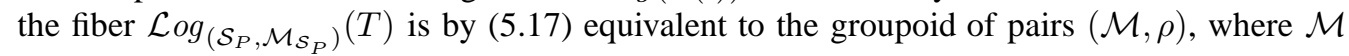
is a fine $\log$ structure on $T$ and $\rho: P \rightarrow \overline{\mathcal{M}}$ is a morphism of sheaves of monoids. The functor $\mathcal{L} \log (\mathcal{S}(l))$ is simply given by

$$
(\mathcal{M}, \rho) \mapsto(\mathcal{M}, \rho \circ l),
$$

and evidently there are no non-trivial automorphisms $(\mathcal{M}, \rho)$ which induce the identity on $(\mathcal{M}, \rho \circ l)$.

To see that $\mathcal{L} \circ g(\mathcal{S}(l))$ is étale, note first that $\log (\mathcal{S}(l))$ is locally of finite presentation since it is a morphism between algebraic stacks locally of finite presentation over $S$; hence is locally of finite presentation by ([4], IV.1.4.3(v)) and the definition of a morphism between algebraic stacks locally of finite presentation ([14], 4.14). Therefore, to show that $\mathcal{L} o g(\mathcal{S}(l))$ is étale it suffices to show that $\mathcal{L} \operatorname{og}(\mathcal{S}(l))$ is formally étale by (4.9(ii)). By (5.17), the infinitesimal lifting criterion of (4.5) amounts to the following: if $i: T_{0} \hookrightarrow T$ is a closed immersion defined by a nilpotent ideal $I, \mathcal{M}$ is a fine $\log$ structure on $T$, and $\varepsilon: Q \rightarrow \overline{\mathcal{M}}$ is a morphism of sheaves of monoids, then any map $\rho: P \rightarrow \iota^{-1} \overline{\mathcal{M}}$ for which $\rho \circ l=\iota^{-1}(\varepsilon)$ can be lifted to a unique map $\tilde{\rho}: P \rightarrow \overline{\mathcal{M}}$ such that $\rho \circ l=\varepsilon$. But this is trivial since the étale sites of $T$ and $T_{0}$ are equivalent by ([5], I.8.3).

Corollary 5.24. - If $S$ is a fine log scheme and $Q \rightarrow \mathcal{M}_{S}$ a chart, then the map $F: \mathcal{S}_{P} \times \mathcal{S}_{Q} \stackrel{\circ}{S} \rightarrow \mathcal{L}^{\circ} g_{S}$ defined by the pullback of the map $l^{*} \mathcal{M}_{\mathcal{S}_{Q}} \rightarrow \mathcal{M}_{\mathcal{S}_{P}}$ on $\mathcal{S}_{P}$ (and (5.8)) is representable and étale.

Proof. - Let $s: \mathcal{S}_{P} \hookrightarrow \log _{\left(\mathcal{S}_{P}, \mathcal{M}_{\mathcal{S}_{P}}\right)}$ be the open immersion (5.9.1). Then the composite $\mathcal{L} \log (\mathcal{S}(l)) \circ s: \mathcal{S}_{P} \rightarrow \mathcal{L}_{\log }\left(\mathcal{S}_{Q}, \mathcal{M}_{\left.\mathcal{S}_{Q}\right)}\right.$ is an étale morphism over $\mathcal{S}_{Q}$ by (5.23), and the map $F$ is simply the base change of this map by the morphism $\stackrel{\circ}{S} \rightarrow \mathcal{S}_{Q}$.

COROLlary 5.25. - Fix a fine log scheme $S$, and let $J$ be the set of triples $(U, \beta, l)$, where $U / S$ is étale, $\beta:\left.Q \rightarrow \mathcal{M}_{S}\right|_{U}$ is a chart, and $l: Q \rightarrow P$ is a morphism of monoids. Then the natural morphism

$$
\tilde{\mathcal{S}}:=\coprod_{(U, \beta, l) \in J} \mathcal{S}_{P} \times \mathcal{S}_{Q} U \rightarrow \mathcal{L} \operatorname{Lg}_{S}
$$

is representable, étale, and surjective.

Remark 5.26. - Suppose $S$ is an fs $\log$ scheme, and let $\mathcal{T}_{\text {or }} \subset \mathcal{L} \mathcal{L}_{S}$ be the substack classifying fs $\log$ schemes over $S$. In general if $\mathcal{M}$ is a fine $\log$ structure on a scheme $X$, then the set of points $x \in X$ for which $\overline{\mathcal{M}}_{\bar{x}}$ is saturated is an open set since (3.5) shows that it is constructible and stable under generization. Therefore, $\mathcal{T}$ or $r_{S}$ is an open substack of $\mathcal{L}_{o g_{S}}$; in particular $\mathcal{T}$ or ${ }_{S}$ is an algebraic stack. If $\mathcal{U} \subset \tilde{\mathcal{S}}$ denotes the open substack obtained by considering only those triples $(U, \beta, l)$ for which $P$ is saturated and torsion free, then the map $\mathcal{U} \rightarrow \mathcal{T}$ or $_{S}$ is surjective by (2.2). Thus $\mathcal{T}$ or $_{S}$ admits an étale cover by toric stacks in the sense of [13].

Remark 5.27. - In fact, Remark (5.26) enables one to prove that $\mathcal{T}$ or ${ }_{S}$ is algebraic without appealing to (A.2) used in the proof of (1.1). Indeed as mentioned in (3.1) the proof that the diagonal

$$
\Delta_{\mathcal{T}_{\text {or }}}: \mathcal{T} \text { or }_{S} \rightarrow \mathcal{T} \text { or }_{S} \times_{S} \mathcal{T}_{\text {or }}{ }_{S}
$$


is representable does not require the use of (A.2). Moreover, when $P$ is fs and torsion free the proof of (5.20) does not require any results from the appendix (as noted in the proof), and so one can construct the étale cover $\mathcal{U} \rightarrow \mathcal{T}$ or $_{S}$ without appealing to results about log structures in the fppf topology. Since $\mathcal{U}$ is an algebraic stack it admits a smooth cover $U \rightarrow \mathcal{U}$ by a scheme, and the composite $U \rightarrow \mathcal{T}$ or $_{S}$ is then a smooth cover of $\mathcal{T}$ or ${ }_{S}$.

Example 5.28. - Let $k$ be a field of characteristic $p>0$ and let

$$
\rho:\left[\mathbb{A}^{1} / \mathbb{G}_{m}\right] \rightarrow\left[\mathbb{A}^{1} / \mathbb{G}_{m}\right]
$$

be the morphism of stacks over $k$ associated to the maps

$$
\rho_{\mathbb{A}^{1}}: \mathbb{A}^{1} \rightarrow \mathbb{A}^{1}, \quad \rho_{\mathbb{G}_{m}}: \mathbb{G}_{m} \rightarrow \mathbb{G}_{m}
$$

induced by

$$
k[T] \rightarrow k[T], \quad T \mapsto T^{p}, \quad \text { and } \quad k\left[U^{ \pm}\right] \rightarrow k\left[U^{ \pm}\right], \quad U \mapsto U^{p} .
$$

The map $\rho$ is not smooth since there is a commutative diagram

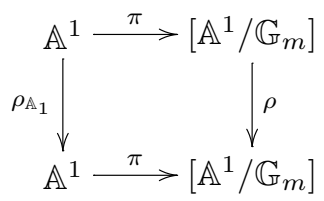

where $\pi$ is smooth but $\rho_{\mathbb{A}^{1}}$ is not. However, if we view $\left[\mathbb{A}^{1} / \mathbb{G}_{m}\right]$ as the log stack $\mathcal{S}_{\mathbb{N}}$ and $\rho$ as the underlying morphism of stacks associated to the morphism of log stacks

$$
\mathcal{S}(\times p):\left(\mathcal{S}_{\mathbb{N}}, \mathcal{M}_{\mathcal{S}_{\mathbb{N}}}\right) \rightarrow\left(\mathcal{S}_{\mathbb{N}}, \mathcal{M}_{\mathcal{S}_{\mathbb{N}}}\right)
$$

induced by multiplication by $p$ on $\mathbb{N}$, then by (5.23) the map $\mathcal{S}(\times p)$ is log étale.

Example 5.29. - Continuing with the preceding example, define

$$
\mathcal{S}:=\left[\mathbb{A}^{1} / \mathbb{G}_{m}\right] \times_{\rho,\left[\mathbb{A}^{1} / \mathbb{G}_{m}\right], \rho}\left[\mathbb{A}^{1} / \mathbb{G}_{m}\right]
$$

Then $\mathcal{S}$ is isomorphic to the stack $\mathcal{S}_{P}$, where $P$ is the quotient of the free monoid generated by two elements $x$ and $y$ modulo the relation $p x=p y$. By (5.23) this stack with its natural log structure is log étale over $k$ (with the trivial log structure). Note however, that $\operatorname{Spec}(k[P])$ with its natural log structure is not log smooth. Indeed even the open set $\operatorname{Spec}\left(k\left[P^{g p}\right]\right) \simeq \mu_{p} \times \mathbb{G}_{m}$ where the $\log$ structure on $\operatorname{Spec}(k[P])$ is trivial is not smooth.

In addition, the underlying stack $\mathcal{S}_{P}$ is not smooth over $k$ either. To see this, let $P^{\prime}$ be the quotient of $\mathbb{N}^{2} \oplus \mathbb{Z}$ by the relation $(p, 0,0)=(0, p, 1)$ and let $\beta: P^{\prime} \rightarrow P$ be the map obtained by sending $(1,0,0)$ to $x,(0,1,0)$ to $y$, and $(0,0,1)$ to 0 . If $\mathcal{M}$ is a fine $\log$ structure on a scheme $X$ and $\gamma: P^{\prime} \rightarrow \overline{\mathcal{M}}$ is a map which fppf locally lifts to a chart, then $\gamma$ factors through $\beta$ since $(0,0,1) \in P^{\prime}$ is a unit. Thus by (5.14) the map $\beta$ induces an isomorphism $\mathcal{S}(\beta): \mathcal{S}_{P} \simeq \mathcal{S}_{P^{\prime}}$. Since $P^{\prime g p} \simeq \mathbb{Z}^{2}$, the map $\operatorname{Spec}\left(k\left[P^{\prime}\right]\right) \rightarrow \mathcal{S}_{P^{\prime}}$ is smooth, and since $\operatorname{Spec}\left(k\left[P^{\prime}\right]\right)$ is not smooth, $\mathcal{S}_{P^{\prime}}$ is not smooth either. 
Finally let $\mathcal{P}$ be a property of morphisms $f: X \rightarrow Y$ of algebraic spaces which is stable under base change and étale local on source and target. That is, for any family of commutative squares

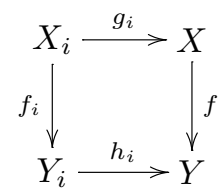

for which the maps

$$
\coprod_{i} g_{i}: \coprod_{i} X_{i} \rightarrow X, \quad \coprod_{i} h_{i}: \coprod_{i} Y_{i} \rightarrow Y
$$

are étale and surjective, the map $f$ has property $\mathcal{P}$ if and only if all the maps $f_{i}$ have property $\mathcal{P}$. For example, $\mathcal{P}$ could be the property of being étale, smooth, or flat. If $F: \mathcal{S}_{1} \rightarrow \mathcal{S}_{2}$ is a representable morphism of algebraic stacks, then it makes sense, by ([14], 3.10.1), to say that $F$ has property $\mathcal{P}$.

Remark 5.30. - If $g: \mathcal{U} \rightarrow \mathcal{V}$ is a representable and étale morphism of algebraic stacks, and if $f: X \rightarrow \mathcal{U}$ is a map from a scheme, then it follows from our assumptions on $\mathcal{P}$ that $f$ has property $\mathcal{P}$ if and only if $g \circ f$ has property $\mathcal{P}$.

We can describe in terms of the stacks $\mathcal{S}_{P}$ what it means for a morphism of log schemes to have property weak $\log (\mathcal{P})$ :

COROLlary 5.31. - A morphism of log schemes $f: X \rightarrow S$ has property weak $\mathcal{L}$ og $(\mathcal{P})$ if and only if étale locally on $S$ and $X$ there exists a chart $\left(Q, P, \beta_{Q}, \beta_{P}, \theta\right)$ for $f$ such that the induced map

$$
\stackrel{\circ}{X} \rightarrow \stackrel{\circ}{S} \times \mathcal{S}_{Q} \mathcal{S}_{P}
$$

has property $\mathcal{P}$. Moreover, if $f$ has property weak $\mathcal{L}$ og $(\mathcal{P})$, then for any chart $\left(Q, P, \beta_{Q}, \beta_{P}, \theta\right)$ the map (5.31.1) has property $\mathcal{P}$.

Proof. - To see the "if" direction, note that by the assumptions on $\mathcal{P}$, we may replace $S$ and $X$ by étale covers, and hence may assume that we have a chart such that (5.31.1) has property $\mathcal{P}$. In this case (5.24) and (5.30) applied to

$$
\stackrel{\circ}{X} \stackrel{h}{\rightarrow} \stackrel{\circ}{S} \times \mathcal{S}_{Q} \mathcal{S}_{P} \stackrel{g}{\rightarrow} \mathcal{L}_{\log }
$$

show that $f$ has property weak $\log (\mathcal{P})$.

To prove the "only if" direction and the second statement, we may assume that we have a chart $\left(Q, P, \beta_{Q}, \beta_{P}, \theta\right)$ for $f$ so that we have a factorization as in (5.31.2). If $f$ has property weak $\log (\mathcal{P})$, then the map $g \circ h$ has property $\mathcal{P}$, and so (5.24) and (5.30) imply that $h$ also has property $\mathcal{P}$.

\section{Acknowledgements}

Relations between logarithmic geometry and algebraic stacks were first investigated by L. Illusie, following a suggestion of L. Lafforgue. We are grateful to Professor Illusie for allowing us to include some of his results in the last Section of this paper, and for enlightening discussions about log geometry. In addition, Professor Illusie gave very helpful comments about earlier versions of this paper. A. Abbes made several important suggestions during the course 
of this work. In particular he suggested (1.1). We also want to thank the referee who gave very detailed and helpful comments. Finally we are grateful to A. Ogus who supervised the author's dissertation which contained many of the results of this paper. Professor Ogus' advice and encouragement has been a great source of inspiration. During the course of this work the author has been partially funded by the Clay Mathematics Institute and by an NSF post-doctoral research fellowship.

\section{Appendix A. Comparison of topologies}

Let $X$ be a scheme, and let $X_{\text {Zar }}\left(\right.$ resp. $X_{\text {et }}, X_{\mathrm{fl}}$ ) denote the small Zariski site (resp. small étale site, big fppf site) ([15], Chapter II, §1). Thus the objects of $X_{\mathrm{fl}}$ are all schemes locally of finite presentation over $X$, and coverings in $X_{\mathrm{f}}$ are given by surjective flat morphisms locally of finite presentation.

We can speak of fine log structures on $X$ with any of these topologies: a log structure is fine if it is locally isomorphic to the $\log$ structure associated to a morphism $P \rightarrow \mathcal{O}_{X}$ from a fine monoid $P$.

Let

$$
X_{\mathrm{fl}} \stackrel{\pi_{1}}{\rightarrow} X_{\mathrm{et}} \stackrel{\pi_{2}}{\rightarrow} X_{\mathrm{Zar}}
$$

be the natural morphisms of ringed topoi. If $\mathcal{M}$ is a pre-log structure on $X_{\mathrm{Zar}}$, then $\pi_{2}^{-1} \mathcal{M}$ can naturally be viewed as a pre-log structure on $X_{\text {et }}$ via the composite

$$
\pi_{2}^{-1} \mathcal{M} \stackrel{\pi_{2}^{-1}(\alpha)}{\longrightarrow} \pi_{2}^{-1} \mathcal{O}_{X_{\mathrm{Zar}}} \rightarrow \mathcal{O}_{X_{\mathrm{et}}}
$$

By taking the associated $\log$ structure, we get for any $\log$ structure $\mathcal{M}$ on $X_{\mathrm{Zar}}$ a log structure

$$
\pi_{2}^{*} \mathcal{M}:=\left(\pi_{2}^{-1} \mathcal{M}\right)^{a}
$$

on $X_{\text {et }}$. Similarly, if $\mathcal{M}$ is a $\log$ structure on $X_{\text {et }}$, then we get a $\log$ structure $\pi_{1}^{*} \mathcal{M}$ on $X_{\mathrm{f}}$. Just as in ([9], 1.4.2), if $\mathcal{M}$ is a pre-log structure on $X_{\text {et }}$ (resp. $\left.X_{\text {Zar }}\right)$, then $\pi_{1}^{*}\left(\mathcal{M}^{a}\right)\left(\right.$ resp. $\left.\pi_{2}^{*}\left(\mathcal{M}^{a}\right)\right)$ is canonically isomorphic to $\left(\pi_{1}^{-1} \mathcal{M}\right)^{a}$ (resp. $\left.\left(\pi_{2}^{-1} \mathcal{M}\right)^{a}\right)$. In particular if $\mathcal{M}$ is a fine log structure on $X_{\text {et }}$ (resp. $X_{\mathrm{Zar}}$ ) then $\pi_{1}^{*} \mathcal{M}$ (resp. $\left.\pi_{2}^{*} \mathcal{M}\right)$ is also fine.

The functor $\pi_{1}^{*}$ (resp. $\pi_{2}^{*}$ ) has a right adjoint $\pi_{1 *}^{\log }$ (resp. $\left.\pi_{2 *}^{\log }\right)$. If $(\mathcal{M}, \alpha)$ is a log structure on $X_{\mathrm{fl}}\left(\right.$ resp. $X_{\mathrm{et}}$ ), then $\pi_{1 *}^{\log }(\mathcal{M})$ (resp. $\pi_{2 *}^{\log }(\mathcal{M})$ ) is simply the restriction of $\mathcal{M}$ to $X_{\text {et }}$ (resp. $X_{\mathrm{Zar}}$ ) with map to $\mathcal{O}_{X_{\mathrm{et}}}$ (resp. $\mathcal{O}_{X_{\mathrm{Zar}}}$ ) given by restriction of $\alpha$. The fact that $\left(\pi_{1}^{*}, \pi_{1 *}^{\log }\right)$ (resp. $\left.\left(\pi_{2}^{*}, \pi_{2 *}^{\log }\right)\right)$ are adjoint functors is checked as in ([9], 1.4).

The following theorem summarizes the basic relationship between the categories of log structures in the various topologies (see also $([16], \S 2)$ for a comparison of the étale and Zariski topologies):

THEOREM A.1. - The functor $\pi_{1}^{*}\left(\right.$ resp. $\left.\pi_{2}^{*}\right)$ induces an equivalence between the category of fine log structures on $X_{\mathrm{et}}$ (resp. $X_{\mathrm{Zar}}$ ) and the category of fine log structures on $X_{\mathrm{fl}}$ (resp. fine log structures $\mathcal{M}$ on $X_{\text {et }}$ for which the adjunction map $\pi_{2}^{-1} \pi_{2 *} \overline{\mathcal{M}} \rightarrow \overline{\mathcal{M}}$ is an isomorphism).

Before giving the proof of (A.1), let us note the following corollaries:

Corollary A.2. - Let $S$ be a fine log scheme (with log structure on the étale topology). Then the fibered category $\mathcal{L}_{\log }$ is a stack with respect to the fppf-topology on the category of $\stackrel{\circ}{S}$-schemes. 
Proof. - It follows from (A.1) that $\mathcal{L}_{\mathrm{L}} g_{S}$ is equivalent to the fibered category over the category of $\stackrel{\leftrightarrow}{S}$-schemes whose objects are morphisms

$$
\left(X, \mathcal{M}_{X}\right) \rightarrow\left(\stackrel{\circ}{S}, \pi_{1}^{*} \mathcal{M}_{S}\right)
$$

where $\mathcal{M}_{X}$ is a fine log structure on $X_{\mathrm{ff}}$. Since log structures and morphisms of log structures in the fppf topology may be constructed fppf-locally it follows that $\mathcal{L}_{o g}$ is a stack with respect to the fppf topology.

Corollary A.3. - Let $S$ be a scheme and $P$ be a fine monoid. Denote by $\mathcal{S}_{P}^{\prime}$ the fibered category over the category of $S$-schemes whose fiber over a scheme $T$ is the groupoid of pairs $(\mathcal{N}, \gamma)$, where $\mathcal{N}$ is a fine log structure on $T_{\text {et }}$ and $\gamma: P \rightarrow \overline{\mathcal{N}}$ is a map which fppf locally on $T$ lifts to a chart. Then $\mathcal{S}_{P}^{\prime}$ is a stack with respect to the fppf topology on the category of $S$-schemes.

Proof. - If $\mathcal{M}$ is a fine $\log$ structure on the étale site of a scheme $X$, then for any fine monoid $P$, to give a map $\gamma_{\mathrm{et}}: P \rightarrow \overline{\mathcal{M}}$ of étale sheaves is equivalent to giving a map of fppf sheaves $\gamma_{\mathrm{fl}}: P \rightarrow \pi_{1}^{-1} \overline{\mathcal{M}}$ since by adjunction

$$
\operatorname{Hom}_{X_{\mathrm{fl}}}\left(P, \pi_{1}^{-1} \overline{\mathcal{M}}\right)=\operatorname{Hom}_{X_{\mathrm{et}}}\left(P, \pi_{1 *} \pi_{1}^{-1} \overline{\mathcal{M}}\right)=\operatorname{Hom}_{X_{\mathrm{et}}}(P, \overline{\mathcal{M}}),
$$

where the last equality follows from ([3], VIII.9.2). We claim that $\gamma_{\mathrm{et}}$ lifts fppf locally to a chart for $\mathcal{M}$ if and only if $\gamma_{\mathrm{ff}}$ lifts fppf locally to a chart for $\pi_{1}^{*} \mathcal{M}$. The "only if" direction is clear. To see the "if" direction, we may by (2.3) assume that we have a lifting $\tilde{\gamma}_{\text {et }}: P \rightarrow \mathcal{M}$. Let $\iota: \mathcal{N} \rightarrow \mathcal{M}$ be the resulting morphism of fine log structures on $X_{\text {et }}$. By (2.4(i)) (which also holds for fppf log structures by the same argument) and the assumption that $\gamma_{\mathrm{fl}}$ lifts fppf locally to a chart for $\pi_{1}^{*} \mathcal{M}$, the map $\pi_{1}^{*}: \pi_{1}^{*} \mathcal{N} \rightarrow \pi_{1}^{*} \mathcal{M}$ is an isomorphism. Then by (A.1), the map $\iota$ is an isomorphism as well and so $\gamma_{\text {et }}$ lifts fppf locally to a chart.

It follows from this discussion and (A.1) that $\mathcal{S}_{P, \mathrm{fl}}^{\prime}$ is equivalent to the fibered category over the category of $S$-schemes whose fiber over a scheme $T$ is the groupoid of pairs $(\mathcal{N}, \gamma)$, where $\mathcal{N}$ is a fine $\log$ structure on $T_{\mathrm{f}}$ and $\gamma: P \rightarrow \overline{\mathcal{N}}$ is a morphism which fppf locally lifts to a chart. Since fppf sheaves and morphisms between them may be constructed fppf locally it follows that $\mathcal{S}_{P, \mathrm{fl}}^{\prime}$ is a stack with respect to the fppf topology.

Corollary A.4. - Let $X$ be a scheme and let $\mathcal{M}_{1}$ and $\mathcal{M}_{2}$ be two fine log structures on

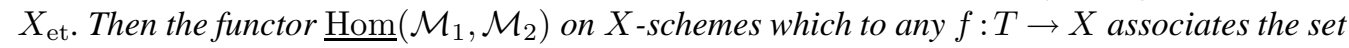
of morphisms of log structures $f^{*} \mathcal{M}_{1} \rightarrow f^{*} \mathcal{M}_{2}$ is a sheaf with respect to the fppf topology.

Proof. - By (A.1), the functor $\underline{\operatorname{Hom}}\left(\mathcal{M}_{1}, \mathcal{M}_{2}\right)$ is isomorphic to the functor

$$
\underline{\operatorname{Hom}}\left(\pi_{1}^{*} \mathcal{M}_{1}, \pi_{1}^{*} \mathcal{M}_{2}\right)
$$

which to any $f: T \rightarrow X$ associates the set of morphisms $f^{*}\left(\pi_{1}^{*} \mathcal{M}_{1}\right) \rightarrow f^{*}\left(\pi_{1}^{*} \mathcal{M}_{2}\right)$. Since morphisms between fppf sheaves may be constructed fppf locally the result follows.

Corollary A.5. - Let $X$ be a scheme and $X^{\prime} \rightarrow X$ an fppf cover of $X$. Then there is a natural equivalence of categories between the category of fine log structures on $X_{\mathrm{et}}$, and the category of pairs $\left(\mathcal{M}^{\prime}, \sigma\right)$, where $\mathcal{M}^{\prime}$ is a fine log structure on $X_{\text {et }}^{\prime}$ and $\sigma: \operatorname{pr}_{1}^{*} \mathcal{M}^{\prime} \rightarrow \operatorname{pr}_{2}^{*} \mathcal{M}^{\prime}$ is an isomorphism on $X^{\prime} \times_{X} X^{\prime}$ satisfying the cocycle condition

$$
\operatorname{pr}_{13}^{*}(\sigma)=\operatorname{pr}_{23}^{*}(\sigma) \circ \operatorname{pr}_{12}^{*}(\sigma)
$$

on $X^{\prime} \times_{X} X^{\prime} \times_{X} X^{\prime}$ 
Proof. - By (A.1), the category of fine $\log$ structures on $X_{\text {et }}$ is equivalent to the category of fine log structures on $X_{\mathrm{fl}}$, and the category of pairs $\left(\mathcal{M}^{\prime}, \sigma\right)$ as in the corollary is equivalent to the category of pairs $\left(\mathcal{M}_{\mathrm{fl}}^{\prime}, \sigma_{\mathrm{fl}}\right)$, where $\mathcal{M}_{\mathrm{fl}}^{\prime}$ is a fine log structure on $X_{\mathrm{fl}}^{\prime}$ and $\sigma_{\mathrm{fl}}: \mathrm{pr}_{1}^{*} \mathcal{M}_{\mathrm{fl}}^{\prime} \simeq \operatorname{pr}_{2}^{*} \mathcal{M}_{\mathrm{fl}}^{\prime}$ is an isomorphism of log structures on $\left(X^{\prime} \times_{X} X^{\prime}\right)_{\mathrm{fl}}$ satisfying the cocycle condition. From this the result follows.

We now begin the proof of (A.1) which will be in several steps ((A.6)-(A.11)).

Proposition A.6. - Suppose $u: X^{\prime} \rightarrow X$ is a faithfully flat morphism of finite presentation, and that $\mathcal{M}$ is an integral log structure ([9], 2.2) on $X_{\mathrm{et}}$ (resp. $\left.X_{\mathrm{Zar}}\right)$. Let $\mathrm{pr}_{i}(i=1,2)$ be the two projections $X^{\prime} \times_{X} X^{\prime} \rightrightarrows X^{\prime}$, and let $\tilde{u}=u \circ \mathrm{pr}_{1}=u \circ \mathrm{pr}_{2}$. Then the sequence

$$
\Gamma(X, \mathcal{M}) \rightarrow \Gamma\left(X^{\prime}, u^{*} \mathcal{M}\right) \rightrightarrows \Gamma\left(X^{\prime} \times_{X} X^{\prime}, \tilde{u}^{*} \mathcal{M}\right)
$$

is exact.

Proof. - It is known that the sequence

$$
\Gamma(X, \overline{\mathcal{M}}) \rightarrow \Gamma\left(X^{\prime}, u^{-1} \overline{\mathcal{M}}\right) \rightrightarrows \Gamma\left(X^{\prime} \times_{X} X^{\prime}, \tilde{u}^{-1} \overline{\mathcal{M}}\right)
$$

is exact (in the étale topology this is ([3], VIII.9.2) and a similar argument gives the result in the Zariski topology). Thus if $m_{1}, m_{2} \in \Gamma(X, \mathcal{M})$ are two sections which map to the same element in $\Gamma\left(X^{\prime}, u^{*} \mathcal{M}\right)$, then there exists a unique unit $\gamma \in \Gamma\left(X, \mathcal{O}_{X}^{*}\right)$ such that $\lambda(\gamma)+m_{1}=m_{2}$. Since the map $\Gamma\left(X, \mathcal{O}_{X}^{*}\right) \rightarrow \Gamma\left(X^{\prime}, \mathcal{O}_{X^{\prime}}^{*}\right)$ is injective, the images of $m_{1}$ and $m_{2}$ in $\Gamma\left(X^{\prime}, u^{*} \mathcal{M}\right)$ are equal if and only if $\gamma=1$ and $m_{1}=m_{2}$. Hence $\Gamma(X, \mathcal{M}) \rightarrow \Gamma\left(X^{\prime}, u^{*} \mathcal{M}\right)$ is injective.

Next suppose $m^{\prime} \in \Gamma\left(X^{\prime}, u^{*} \mathcal{M}\right)$ is an element for which $\operatorname{pr}_{1}^{*} m^{\prime}=\operatorname{pr}_{2}^{*} m^{\prime}$. To prove that $m^{\prime}$ is in the image of $\Gamma(X, \mathcal{M})$, we may, by the injectivity shown above, replace $X$ by an étale cover (resp. Zariski cover). By the exactness of (A.6.1), the image of $m^{\prime}$ in $\Gamma\left(X^{\prime}, u^{-1} \overline{\mathcal{M}}\right)$ is contained in $\Gamma(X, \overline{\mathcal{M}})$. Therefore, after perhaps replacing $X$ by an étale cover (resp. Zariski cover), we may assume that there exists a Section $m \in \Gamma(X, \mathcal{M})$ whose image in $\Gamma\left(X^{\prime}, u^{-1} \overline{\mathcal{M}}\right)$ is equal to the image of $m^{\prime}$. Since $\mathcal{M}$ is integral, there exists a unique unit $\gamma \in \Gamma\left(X^{\prime}, \mathcal{O}_{X^{\prime}}^{*}\right)$ such that $\lambda(\gamma)+m=m^{\prime}$. Moreover, since both $m$ and $m^{\prime}$ are in the equalizer of $\operatorname{pr}_{1}^{*}$ and $\operatorname{pr}_{2}^{*}, \gamma$ is in the equalizer of the two maps

$$
\Gamma\left(X^{\prime}, \mathcal{O}_{X^{\prime}}^{*}\right) \rightrightarrows \Gamma\left(X^{\prime} \times_{X} X^{\prime}, \mathcal{O}_{X^{\prime} \times_{X} X^{\prime}}^{*}\right) .
$$

By descent theory $\gamma \in \Gamma\left(X, \mathcal{O}_{X}^{*}\right)$, and hence $m^{\prime} \in \Gamma(X, \mathcal{M})$.

Corollary A.7. - If $\mathcal{M}$ is an integral log structure on $X_{\text {et }}\left(\right.$ resp. $X_{\text {Zar }}$ ), then $\pi_{1}^{*} \mathcal{M}$ (resp. $\left.\pi_{2}^{*} \mathcal{M}\right)$ is the log structure which to any morphism of finite presentation (resp. étale) $f: X^{\prime} \rightarrow X$ associates $\Gamma\left(X^{\prime}, f^{*} \mathcal{M}\right)$. In particular if $\mathcal{M}$ is an integral log structure on $X_{\text {et }}$ (resp. $\left.X_{\mathrm{Zar}}\right)$, then the natural map $\mathcal{M} \rightarrow \pi_{1 *}^{\text {log }} \pi_{1}^{*} \mathcal{M}$ (resp. $\mathcal{M} \rightarrow \pi_{2 *}^{\text {log }} \pi_{2}^{*} \mathcal{M}$ ) is an isomorphism.

COROLLARY A.8. - The restriction of $\pi_{1}^{*}\left(\right.$ resp. $\left.\pi_{2}^{*}\right)$ to the category of integral log structures on $X_{\mathrm{et}}$ (resp. $\left.X_{\mathrm{Zar}}\right)$ is fully faithful.

Proof. - If $\mathcal{M}_{1}$ and $\mathcal{M}_{2}$ are integral log structures on $X_{\text {et }}$ (resp. $X_{\text {Zar }}$ ), then

$$
\operatorname{Hom}\left(\pi^{*} \mathcal{M}_{1}, \pi^{*} \mathcal{M}_{2}\right) \simeq \operatorname{Hom}\left(\mathcal{M}_{1}, \pi_{*}^{\log } \pi^{*} \mathcal{M}_{2}\right) \simeq \operatorname{Hom}\left(\mathcal{M}_{1}, \mathcal{M}_{2}\right)
$$

where $\pi$ denotes $\pi_{1}$ (resp. $\pi_{2}$ ). 
Corollary A.9. - Let $X$ be a scheme and $\mathcal{M}$ a fine $\log$ structure on $X_{\mathrm{fl}}$. Then the adjunction map $\pi_{1}^{-1} \pi_{1 *} \overline{\mathcal{M}} \rightarrow \overline{\mathcal{M}}$ is an isomorphism.

Proof. - Let $f: X^{\prime} \rightarrow X$ be a flat cover such that there exist a fine $\log$ structure $\mathcal{N}$ on $X_{\text {et }}^{\prime}$ and an isomorphism $\left.\pi_{1}^{*} \mathcal{N} \simeq \mathcal{M}\right|_{X_{\text {fl }}^{\prime}}$. By (A.8), the tautological descent datum for $\left.\mathcal{M}\right|_{X_{\text {fl }}^{\prime}}$ relative to the morphism $f$ induces descent datum for $\mathcal{N}$ relative to $f$. From this we obtain descent datum for the étale sheaf $\overline{\mathcal{N}}$ relative to $f$, which by ([3], VIII.9.4) is effective. Thus there exists a sheaf of monoids $\mathcal{F}$ on $X_{\text {et }}$ inducing $\overline{\mathcal{N}}$ with its descent datum. Thus $\overline{\mathcal{M}} \simeq \pi_{1}^{-1} \mathcal{F}$, and since $\pi_{1 *} \pi_{1}^{-1} \mathcal{F} \simeq \mathcal{F}$ ([15], III.3.11(b)) the result follows.

Proposition A.10. - Let $X$ be a scheme and $\mathcal{M}$ an integral log structure on $X_{\text {et }}$ (resp. $\left.X_{\mathrm{Zar}}\right)$. Then $\mathcal{M}$ is fine if and only if $\pi_{1}^{*} \mathcal{M}\left(\right.$ resp. $\left.\pi_{2}^{*} \mathcal{M}\right)$ is fine.

Proof. - The "only if" direction was already noted. Now if $\pi_{1}^{*} \mathcal{M}$ (resp. $\pi_{2}^{*} \mathcal{M}$ ) is fine, then there exist a flat (resp. étale) cover $f: X^{\prime} \rightarrow X$ and a fine log structure $\mathcal{M}^{\prime}$ on $X_{\text {et }}^{\prime}$ (resp. $X_{\text {Zar }}^{\prime}$ ) such that $\left.\pi_{1}^{*} \mathcal{M}\right|_{X^{\prime}}\left(\right.$ resp. $\left.\left.\pi_{2}^{*} \mathcal{M}\right|_{X^{\prime}}\right)$ is isomorphic to $\pi_{1}^{*} \mathcal{M}^{\prime}$ (resp. $\pi_{2}^{*} \mathcal{M}^{\prime}$ ). From this and (A.8) we obtain an isomorphism $f^{*} \mathcal{M} \simeq \mathcal{M}^{\prime}$ and hence $f^{*} \mathcal{M}$ is fine. Let $\bar{x} \rightarrow X$ be a geometric point (resp. point) and let $\bar{y} \rightarrow X^{\prime}$ be a geometric point (resp. point) lying over $\bar{x}$. Then

$$
\overline{\mathcal{M}}_{\bar{x}} \simeq\left(f^{-1} \overline{\mathcal{M}}\right)_{\bar{y}} \simeq \overline{\mathcal{M}}_{\bar{y}}
$$

which implies that $\overline{\mathcal{M}}_{\bar{x}}$ is a fine monoid. Choose a finitely generated group $G$ and a map $h: G \rightarrow \mathcal{M}_{\bar{x}}^{g p}$ such that the composite $G \rightarrow \overline{\mathcal{M}}_{\bar{x}}^{g p}$ is surjective. Then by ([9], proof of 2.10) the monoid $P:=h^{-1}\left(\mathcal{M}_{\bar{x}}\right)$ is fine, and so after replacing $X$ by some étale (resp. Zariski) neighborhood of $\bar{x}$ we can find a map $P \rightarrow \mathcal{M}$. Let $\mathcal{N}$ be the log structure associated to $P$ and let $\mathcal{N} \rightarrow \mathcal{M}$ be the induced map. By ([9], 2.10) there exists an étale (resp. Zariski) neighborhood of $\bar{y}$ such that $f^{*} \mathcal{N} \rightarrow f^{*} \mathcal{M}$ is an isomorphism. From this and (A.8) it follows that $\mathcal{N} \rightarrow \mathcal{M}$ is an isomorphism in some neighborhood of $\bar{x}$.

Proposition A.11. - Let $X$ be a scheme and $\mathcal{M}$ a fine log structure on $X_{\mathrm{fl}}$ (resp. $X_{\mathrm{et}}$ such that $\pi_{2}^{-1} \pi_{2 *} \overline{\mathcal{M}} \rightarrow \overline{\mathcal{M}}$ is an isomorphism). Then there exists a pair $(\mathcal{N}, s)$, where $\mathcal{N}$ is a fine log structure on $X_{\mathrm{et}}$ (resp. $\left.X_{\mathrm{Zar}}\right)$ and $s$ is an isomorphism between $\mathcal{M}$ and $\pi_{1}^{*} \mathcal{N}$ (resp. $\left.\pi_{2}^{*} \mathcal{N}\right)$.

Proof. - Let $\pi$ denote $\pi_{1}$ (resp. $\pi_{2}$ ). Define $\mathcal{N}:=\pi_{*}^{\log } \mathcal{M}$, and let $s: \pi^{*} \mathcal{N} \rightarrow \mathcal{M}$ be the map obtained by adjunction. To prove the proposition it suffices to show that $s$ is an isomorphism. Once this is shown, the fact that $\mathcal{N}$ is fine follows from (A.10).

To show that $s$ is an isomorphism, it suffices to show that

$$
\bar{s}: \pi^{-1}(\overline{\mathcal{N}}) \simeq \overline{\left(\pi^{*} \mathcal{N}\right)} \rightarrow \overline{\mathcal{M}}
$$

is an isomorphism, since $s$ is a morphism of integral log structures ([8], 3.2). By (A.9) (resp. by assumption) the map $\pi^{-1} \pi_{*} \overline{\mathcal{M}} \rightarrow \overline{\mathcal{M}}$ is an isomorphism, so to verify that $\bar{s}$ is an isomorphism it suffices to verify that $\pi_{*}(\bar{s}): \overline{\mathcal{N}} \rightarrow \pi_{*} \overline{\mathcal{M}}$ is an isomorphism.

That $\pi_{*}(\bar{s})$ is injective follows from the commutative diagram

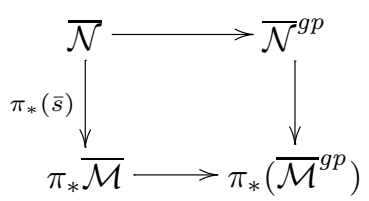


and the snake lemma applied to the following commutative diagram of sheaves on $X_{\text {et }}$ (resp. $\left.X_{\mathrm{Zar}}\right)$ :

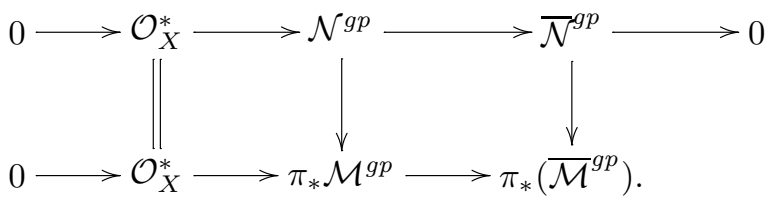

To see that the map $\pi_{*}(\bar{s})$ is surjective, suppose we have a section $\bar{m} \in \pi_{*}(\overline{\mathcal{M}})(U)$ for some étale (resp. Zariski) $U \rightarrow X$. Then the surjectivity of $\pi_{*}(\bar{s})$ is equivalent to the statement that locally in the étale (resp. Zariski) topology on $U$ there exists a lifting of $\bar{m}$ to $\mathcal{M}$. In other words, we have to show that the sheaf $S$ on $U_{\text {fl }}$ (resp. $U_{\text {et }}$ ) defined by

$$
V \mapsto\{\text { liftings of } \bar{m} \text { to } \mathcal{M}(V)\}
$$

admits a section locally in the étale (resp. Zariski) topology. But $S$ is naturally a $\mathbb{G}_{m}$-torsor, so this follows from the fact that any $\mathbb{G}_{m}$-torsor in the flat topology can be trivialized locally in the Zariski topology ([5], XI.5.1).

Example A.12. - Suppose $R$ is a complete discrete valuation ring and $E / R$ a proper regular scheme whose closed fiber $j: E_{0} \subset E$ is a divisor with normal crossings and whose generic fiber is smooth. Suppose that $E_{0}$ is an irreducible nodal genus 1 curve, and let $\mathcal{M}_{E}$ be the log structure on $E_{\text {et }}$ defined by the divisor $E_{0}$ ([9], 1.5.1). Then we claim that $\mathcal{M}_{E}$ is not induced by a $\log$ structure on $E_{\mathrm{Zar}}$. In fact even $\mathcal{M}_{E_{0}}:=j^{*} \mathcal{M}_{E}$ is not induced by a Zariski log structure on $E_{0}$. To see this let $p: \tilde{E}_{0} \rightarrow E_{0}$ be the normalization of $E_{0}$, and note that there is an isomorphism $p_{*} \mathbb{Z} \simeq \overline{\mathcal{M}}_{E_{0}}^{g p}$ ([8], 11.5). Now if $U \subset E_{0}$ is an open set, then $p^{-1}(U) \subset \tilde{E}_{0}$ is connected and hence $\left(p_{*} \mathbb{Z}\right)(U)=\mathbb{Z}$. Thus the restriction of $\overline{\mathcal{M}}_{E_{0}}^{g p}$ to $E_{0, \text { Zar }}$ is the constant sheaf $\mathbb{Z}$, and if $\bar{p} \rightarrow E_{0}$ denotes a geometric point mapping to the node, then the map

$$
\mathbb{Z} \simeq\left(\pi_{2}^{-1} \pi_{2 *} \overline{\mathcal{M}}_{E_{0}}^{g p}\right)_{\bar{p}} \rightarrow \overline{\mathcal{M}}_{E_{0}, \bar{p}}^{g p} \simeq \mathbb{Z}^{2}
$$

is the diagonal map. In particular, the map $\pi_{2}^{-1} \pi_{2 *} \overline{\mathcal{M}}_{E_{0}} \rightarrow \overline{\mathcal{M}}_{E_{0}}$ is not an isomorphism.

\section{REFERENCES}

[1] ARTIN M., Versal deformations and algebraic stacks, Invent. Math. 27 (1974) 165-189.

[2] Artin M., Bertin J.E., Demazure M., Gabriel P., Grothendieck A., Raynaud M., Serre J.-P., Schémas en groupes. Fasc. 1: Exposés 1 à 4, Institut des Hautes Études Scientifiques, Paris, $1963 / 1964$.

[3] Artin M., Grothendieck A., Verdier J.-L., Théorie des topos et cohomologie étale des schémas, in: Artin M., Grothendieck A., Verdier J.L. (Eds.), Séminaire de géométrie algébrique du Bois-Marie 1963-1964 (SGA 4), in: Lecture Notes in Mathematics, Vols. 269, 270, 305, SpringerVerlag, Berlin, 1972, Avec la collaboration de N. Bourbaki, P. Deligne et B. Saint-Donat.

[4] Dieudonné J., Grothendieck A., Éléments de géométrie algébrique, no. 4, 8, 11, 17, 20, 24, 28, 32, Inst. Hautes Études Sci. Publ. Math., Paris, 1961-1967.

[5] GRothendiecK A., Revêtements étale et groupe fondamental, in: Lecture Notes in Mathematics, vol. 224, Springer-Verlag, Berlin, 1971.

[6] Hartshorne R., Algebraic Geometry, in: Graduate Texts in Mathematics, vol. 52, Springer-Verlag, New York, 1977. 
[7] Illusie L., Logarithmic spaces (according to K. Kato), in: Barsotti Symposium in Algebraic Geometry (Abano Terme, 1991), Academic Press, San Diego, CA, 1994, pp. 183-203.

[8] Kato F., Log smooth deformation theory, Tôhoku Math. J. (2) 48 (3) (1996) 317-354.

[9] KАто K., Logarithmic structures of Fontaine-Illusie, in: Algebraic Analysis, Geometry, and Number Theory (Baltimore, MD, 1988), Johns Hopkins Univ. Press, Baltimore, MD, 1989, pp. 191-224.

[10] KАто K., Logarithmic structures of Fontaine-Illusie II: Logarithmic flat topology (incomplete) preprint, 1991.

[11] KaTO K., Log flat morphisms and neat charts, manuscript notes, 1991.

[12] Knutson D., Algebraic Spaces, in: Lecture Notes in Mathematics, vol. 203, Springer-Verlag, Berlin, 1971.

[13] Lafforgue L., Chtoucas de Drinfeld et correspondance de Langlands, Invent. Math. 147 (2002) $1-241$.

[14] Laumon G., Moret-Bailly L., Champs algébriques, Ergeb. Math. 39 (2000).

[15] Milne J.S., Étale Cohomology, Princeton University Press, Princeton, NJ, 1980.

[16] NizIOL W., Toric singularities: log-blow-ups and global resolutions, preprint, 1999.

[17] OGus A., Lectures on logarithmic algebraic geometry, TeXed notes, 2001.

[18] OLsson M., Log algebraic stacks and moduli of log schemes, PhD Thesis, UC Berkeley, May 2001.

[19] OLSSON M., Crystalline cohomology of schemes over algebraic stacks, preprint, 2002.

[20] Olsson M., Universal log structures on semi-stable varieties, Tôhoku Math. J. 55 (2003) 397-438.

(Manuscrit reçu le 18 juillet 2002 ; accepté le 21 novembre 2002.)

\footnotetext{
Martin C. Olsson

Department of Mathematics,

Massachusetts Institute of Technology,

Cambridge, MA 02139, USA

E-mail: molsson@math.mit.edu
} 